\title{
Evaluierung des SOTOS \\ (Silent Operating Theatre Optimisation System) \\ bei roboterassistierten Operationen in der Urologie
}

\author{
INAUGURAL-DISSERTATION \\ zur Erlangung des Doktorgrades \\ der Medizinischen Fakultät der \\ Georg-August-Universität zu Göttingen
}

vorgelegt von

Conrad Leitsmann

aus

Zwickau

Göttingen 2020 
Dekan:

Referent/in

Ko-Referent/in:

Drittreferent/in:
Prof. Dr. med. W. Brück

Prof. Dr. med. L. Trojan

Prof. Dr. med. A. Bräuer

Datum der mündlichen Prüfung: 01.04.2021 
Hiermit erkläre ich, die Dissertation mit dem Titel "Evaluierung des SOTOS (Silent Operating Theatre Optimisation System) bei roboterassistierten Operationen in der Urologie" eigenständig angefertigt und keine anderen als die von mir angegebenen Quellen und Hilfsmittel verwendet zu haben.

Göttingen, den

(Unterschrift) 
Die Daten, auf denen die vorliegende Arbeit basiert, wurden zur Publikation eingereicht:

Leitsmann C, Uhlig A, Popeneciu IV, Boos M, Ahyai S, Schmid M, Wachter R, Trojan L, Friedrich M (2020): The Silent Operation Theatre Optimisation System (SOTOS) to reduce noise pollution during da Vinci robot-assisted laparoscopic radical prostatectomy. J Robot Surg. Aug, 1-9. 


\section{Inhaltsverzeichnis}

Abbildungsverzeichnis ............................................................................................

Tabellenverzeichnis ....................................................................................... III

Abkürzungsverzeichnis .................................................................................

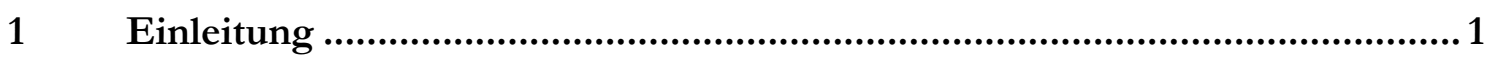

1.1 Das Prostatakarzinom: Epidemiologie und Therapie ........................................................... 1

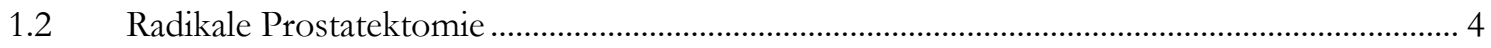

1.3 Da-Vinci-Operationsverfahren für die radikale Prostatektomie .................................................. 5

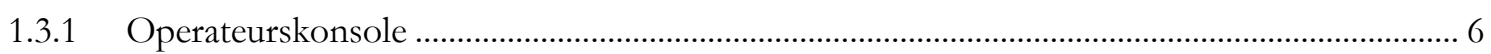

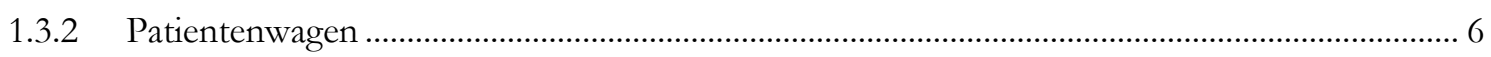

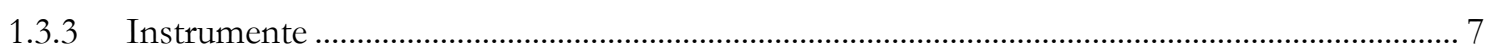

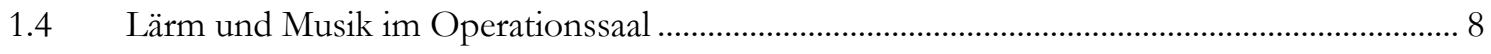

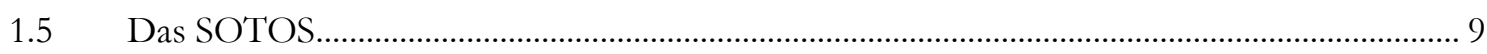

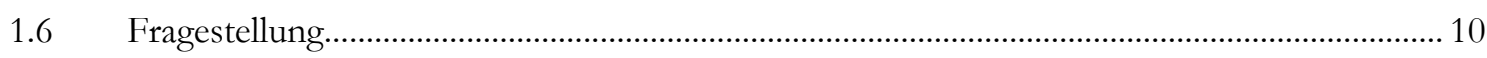

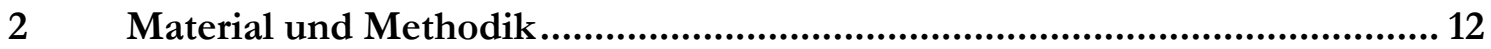

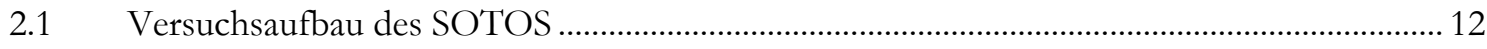

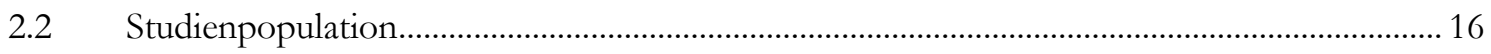

2.3 Erfassung der klinischen Daten der jeweiligen Operationen ................................................... 17

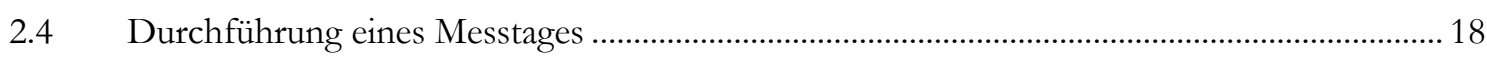

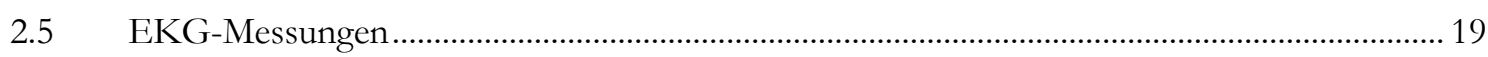

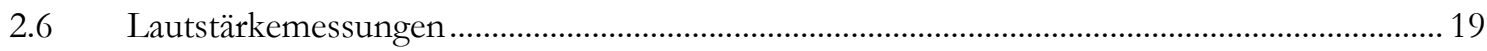

2.7 Auswertung in Bezug auf den Anästhesisten ..................................................................... 20

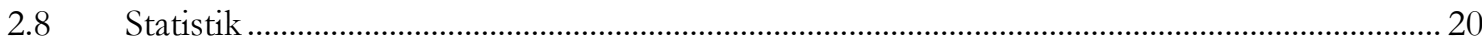

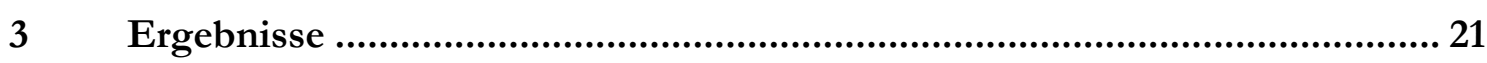

3.1 Klinisch-onkologische Daten und Operationsparameter..................................................... 21

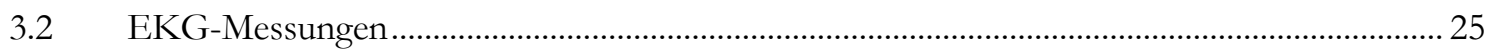

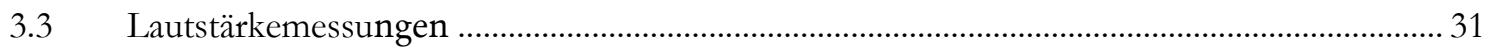

3.3.1 Lautstärkemessungen über die gesamte Operation an beiden Messorten................................. 31

3.3.2 Messergebnisse des Schalldruckpegels an vier verschiedenen Messzeitpunkten..................... 32

3.3.3 Messergebnisse des Schalldruckes an vier verschiedenen Messzeitpunkten ............................. 35

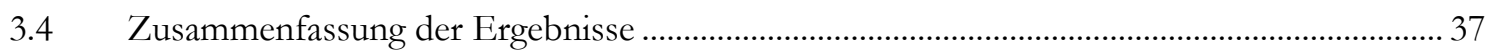

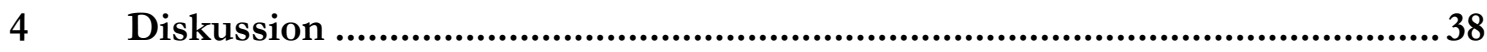

4.1 Vergleichbarkeit der klinisch-onkologischen Daten und der Operationsparameter................38

4.2 Auswirkungen auf die Stressbelastung der Akteure im Operationssaal.................................... 41

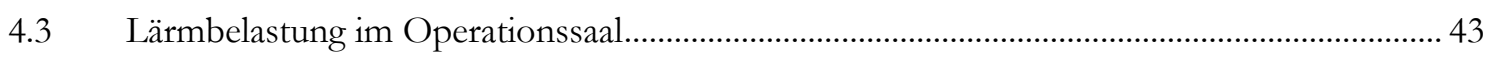

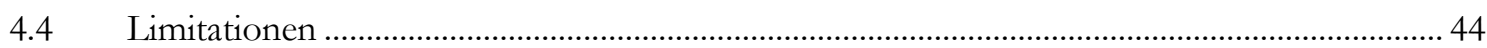

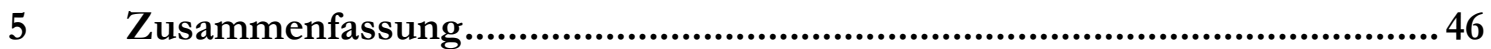

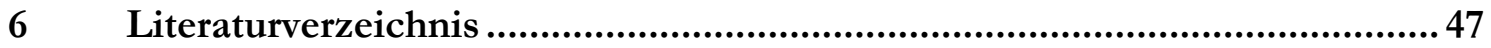




\section{Abbildungsverzeichnis}

Abbildung 1: Altersstandardisierte Neuerkrankungs- und Sterberaten des Prostatakrebses.............. 1

Abbildung 2: Operationssaal mit Da-Vinci-Operationssystem.............................................................. 5

Abbildung 3: Operateurskonsole des Da-Vinci-Operationssystems....................................................... 6

Abbildung 4: Personenwagen des Da-Vinci-Operationssystems............................................................ 6

Abbildung 5: SOTOS in der Verwendung im Operationssaal............................................................... 10

Abbildung 6: Aufbau des Da-Vinci-Operationssaales...................................................................... 12

Abbildung 7: Standort der Lautstärkemessgeräte zur Messung des Schalldruckpegels ..................... 13

Abbildung 8: Messaufbau während eines Messtages mit Einsatz des SOTOS .................................... 14

Abbildung 9: Instrumentierende Fachkraft trägt eine Funkkombination ............................................ 15

Abbildung 10: OP-Assistent trägt eine kabelgebundene Kommunikations-Kombination .................. 15

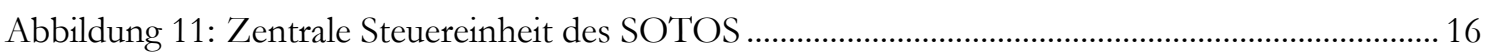

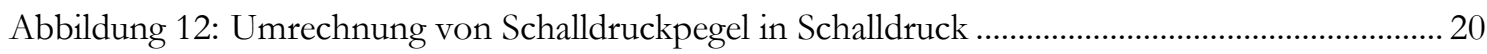

Abbildung 13: Vergleich des Alters der Studienpopulationen ............................................................... 22

Abbildung 14: Vergleich der mittleren Herzfrequenzen der Gesamtkohorte ......................................... 26

Abbildung 15: Vergleich der mittleren Herzfrequenzen der einzelnen OP-Funktionen ..................... 26

Abbildung 16: Vergleich der minimalen Herzfrequenzen der einzelnen OP-Funktionen .................. 27

Abbildung 17: Vergleich der maximalen Herzfrequenzen der einzelnen OP-Funktionen ................. 28

Abbildung 18: Vergleich der mittleren Herzfrequenzen der einzelnen Operateure getrennt. ........... 29

Abbildung 19: Vergleich der minimalen Herzfrequenzen der einzelnen Operateure getrennt.......... 29

Abbildung 20: Vergleich der maximalen Herzfrequenzen der einzelnen Operateure getrennt.......... 30

Abbildung 21: Vergleich der Schalldruckpegel zwischen den beiden Studiengruppen ........................ 31

Abbildung 22: Vergleich des Schalldruckes zwischen den beiden Studiengruppen.............................. 32

Abbildung 23: Vergleich der gemessenen Schalldruckpegel am OP-Feld zu vier verschiedenen

Messzeitpunkten für die beiden Studienarme ………………………………………...... 33

Abbildung 24: Vergleich der gemessenen Schalldruckpegel an der Konsole zu vier

verschiedenen Messzeitpunkten für die beiden Studienarme .....

Abbildung 25: Vergleich des Schalldrucks gemessen am OP-Feld zu den vier verschiedenen

Messzeitpunkten während der Operation zwischen den beiden Studienarmen.......... 35

Abbildung 26: Vergleich des Schalldrucks gemessen an der Konsole zu vier verschiedenen

Messzeitpunkten während der Operation zwischen den beiden Studienarmen..........36 


\section{Tabellenverzeichnis}

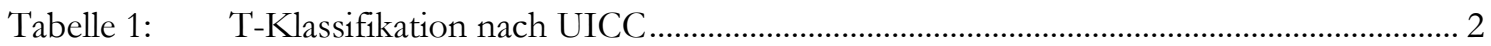

Tabelle 2: $\quad$ Risikogruppen des lokal begrenzten Prostatakarzinoms nach D'Amico ........................ 3

Tabelle 3: Vergleich der präoperativen klinischen Parameter der Studienarme ............................. 22

Tabelle 4: Vergleich der präoperativen onkologischen Parameter der Studienarme .................... 23

Tabelle 5: Vergleich der intraoperativen Parameter der Studienarme............................................ 24

Tabelle 6: Vergleich der postoperativen klinischen Parameter der Studienarme ...........................24

Tabelle 7: Vergleich der postoperativ erhobenen onkologischen Parameter der

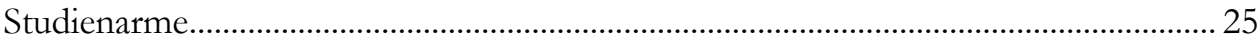

Tabelle 8: Vergleich der gemessenen Schalldruckpegel am OP-Feld zu vier verschiedenen Messzeitpunkten für die beiden Studienarme ...................................................................... 33

Tabelle 9: Vergleich der gemessenen Schalldruckpegel an der Konsole zu vier verschiedenen Messzeitpunkten für die beiden Studienarme mit der Differenz zwischen Kontroll- und SOTOS-Gruppe .34

Tabelle 10: Vergleich des gemessenen Schalldrucks am OP-Feld zu vier verschiedenen Messzeitpunkten für die beiden Studienarme mit der Differenz zwischen Kontroll- und SOTOS-Gruppe

Tabelle 11: Vergleich des gemessenen Schalldrucks an der Konsole zu vier verschiedenen Messzeitpunkten für die beiden Studienarme mit der Differenz zwischen Kontroll- und SOTOS-Gruppe 


\section{Abkürzungsverzeichnis}

EKG Elektrokardiogramm

dB (A) Dezibel in der Wichtung A

HIFU Hochintensiver fokussierter Ultraschall

IRE Irreversible Elektroporation

iPSA Initiales prostataspezifisches Antigen

IQA Interquartilsabstand

KLT-R Konzentrations-Leistungs-Test, revidierte Fassung

MRGN Multiresistente gramnegative Stäbchen-Bakterien

MRSA Methicillin-resistenter Staphylococcus aureus

MRT Magnetresonanztomographie

PC Personal Computer

PSA Prostataspezifisches Antigen

pTNM Postoperatives Tumorstadium

RPE Radikale Prostatektomie

SOTOS Silent Operating Theatre Optimisation System

TNM Tumor, Nodus und Metastasen

TUR-P Transurethrale Resektion der Prostata

TRUS Transrektaler Ultraschall

UICC Union Internationale Contre le Cancer

UMG Universitätsmedizin Göttingen

USB Universal Serial Bus

VRE Vancomycin-resistente Enterokokken 


\section{Einleitung}

\subsection{Das Prostatakarzinom: Epidemiologie \& Therapie}

Das Prostatakarzinom ist die häufigste Krebserkrankung des Mannes in Deutschland mit 59.620 registrierten Neuerkrankungen im Jahre 2013 (Barnes et al. 2016). Gegenwärtig liegt das durchschnittliche Erkrankungsalter für ein Prostatakarzinom bei 71 Jahren (Barnes et al. 2016). Circa 40 \% der männlichen Bevölkerung in den westlichen Industrieländern tragen das Risiko, im Laufe ihres Lebens an einem Prostatakarzinom zu erkranken. Symptome werden jedoch nur $10 \%$ der Männer entwickeln und lediglich $3 \%$ werden daran versterben (Bott et al. 2003).

Bezüglich der Mortalität liegt das Prostatakarzinom an dritter Stelle der Krebssterbefälle in Deutschland. So starben im Jahr 2013 13.408 Männer an dieser Erkrankung. Die 5-JahresÜberlebensrate lag bei $93 \%$, die 10-Jahres-Überlebensrate bei $91 \%$ (Barnes et al. 2016).

Abb.1 zeigt den Trend von Inzidenz und Mortalität, geordnet nach Alterskohorten der letzten 15 Jahre (Barnes et al. 2016).

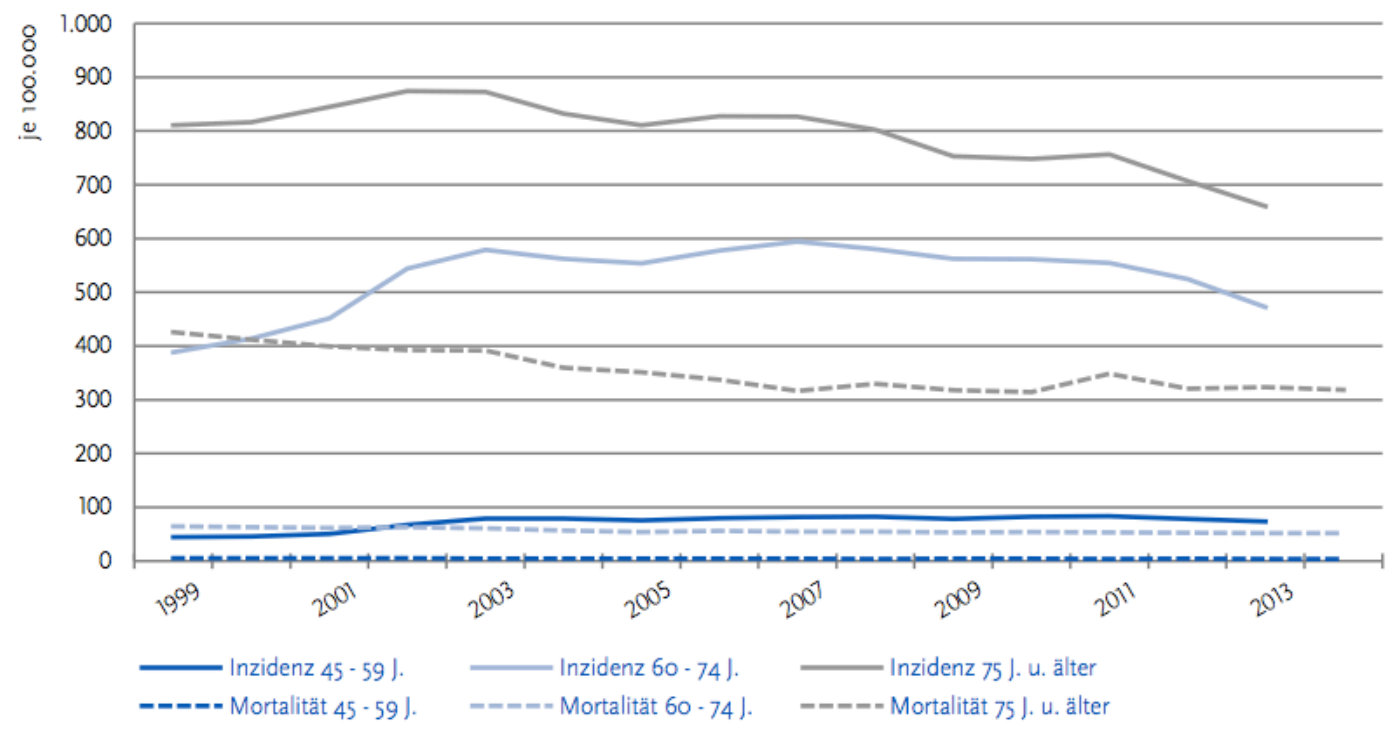

Abbildung 1: Altersstandardisierte Neuerkrankungs- und Sterberaten, Prostatakrebses (ICD-10 C61) nach Altersgruppen, Deutschland, 1999-2014. Quelle: Barnes et al. 2016. (Verwendung der Abbildung mit freundlicher Genehmigung des Robert-Koch-Institutes Hrsg.)

Aufgrund der zu erwartenden zunehmenden Erkrankungsrate bleibt das Management zur Bekämpfung des Prostatakarzinoms ein relevantes onkologisches Thema. Den Schwerpunkt bilden dabei vor allem die Früherkennung bzw. Diagnose und die Therapie ab.

Insbesondere der Anteil der frühen Stadien des Prostatakarzinoms steigt. Durch die Bestimmung des Tumormarkers prostataspezifisches Antigen (PSA) ist diese Stadienverschiebung erklärbar. 
Die Diagnose des Prostatakarzinoms erfolgt meist über ein Screening. Das Screening empfiehlt in Abhängigkeit vom altersabhängigen PSA-Wert und der digital-rektalen Untersuchung bei Verdacht eine Sicherung. Letztendlich geschieht die Sicherung eines Prostatakarzinoms in aller Regel durch eine transrektale sonographisch gesteuerte Biopsie. Zehn bis zwölf Gewebezylinder werden bioptisch entnommen (Leitlinie Prostatakarzinom 2019). Dieses Vorgehen wird von der deutschen (Leitlinie Prostatakarzinom 2019) sowie von den internationalen Fachgesellschaften (Mottet et al. 2017) empfohlen.

Die Untersuchung der Stanzzylinder gibt Auskünfte bezüglich des Gleason-Score und der klinischen Tumorklassifikation (Bostwick et al. 2000; Epstein et al. 1996; Kattan und Eastham 2003; Leitlinie Prostatakarzinom 2019; Lopez-Beltran et al. 2006; Makarov et al. 2007; Partin et al. 2001; Roehl et al. 2004; Svanholm und Mygind 1985).

Tabelle 1: T-Klassifikation (Primärtumor) nach Union Internationale Contre le Cancer (UICC, Wittekind 2017).

\begin{tabular}{|l|l|}
$\begin{array}{l}\text { T1 } \\
\text { (nicht tast- oder } \\
\text { sichtbarer Tumor) }\end{array}$ & $\begin{array}{l}\text { T1a: Zufälliger histologischer Befund in }<5 \% \text { der Resektionsspäne } \\
\text { nach transurethraler Resektion der Prostata (TUR-P) }\end{array}$ \\
$\begin{array}{l}\text { T1b: Zufälliger histologischer Befund in }>5 \% \text { der Resektionsspäne } \\
\text { nach TUR-P } \\
\text { T1c: Nach Stanzbiopsie und nicht tastbarer Tumor }\end{array}$ \\
\hline $\begin{array}{l}\text { T2 } \\
\text { (tastbarer Tumor, } \\
\text { begrenzt auf die } \\
\text { Prostata) }\end{array}$ & $\begin{array}{l}\text { 2a: } \leq \text { Hälfte eines Seitenlappens } \\
\text { 2b: Hälfte eines Seitenlappens }\end{array}$ \\
\hline $\begin{array}{l}\text { T3 } \\
\text { 2c: Beide Lappen sind befallen } \\
\text { die Prostatakapsel) }\end{array}$ & $\begin{array}{l}\text { T3a: Extraprostatische Ausbreitung (einseitig oder beidseitig), einge- } \\
\text { schlossen mikroskopisch nachweisbare Infiltration des Blasenhalses }\end{array}$ \\
\hline T4 & $\begin{array}{l}\text { Infiltration von Nachbarorganen wie Harnblase, Rektum, Schließ- } \\
\text { muskel oder Beckenboden und/oder der Tumor ist an der Becken- } \\
\text { wand fixiert }\end{array}$ \\
\hline
\end{tabular}

Die Therapie des Prostatakarzinoms hängt entscheidend vom Vorhandensein von Fernmetastasen, dem Alter, Komorbiditäten und der Risikogruppe ab. Nach klinischen Angaben sowie der histopathologischen Untersuchung wird das lokal begrenzte Prostatakarzinom bezüglich der Entwicklung eines Rezidivs nach kurativer Therapie in Risikogruppen eingeteilt (D’Amico et al. 1998, Tabelle 2): 
Tabelle 2: Risikogruppen des lokal begrenzten Prostatakarzinoms nach D'Amico.

\begin{tabular}{|l|l|l|l|}
\hline Risikogruppe & PSA-Serumwert $(\mathrm{ng} / \mathrm{ml})$ & Gleason-Score & cT-Stadium $(T N M)$ \\
\hline Low-Risk & $\leq 10$ & 6 & $1 \mathrm{c} / 2 \mathrm{a}$ \\
\hline Intermediate-Risk & $>10-20$ & 7 & $2 \mathrm{~b}$ \\
\hline High-Risk & $>20$ & $\geq 8$ & $2 \mathrm{c}$ \\
\hline
\end{tabular}

Nachfolgend werden die verschiedenen Therapieoptionen nach den Leitlinien der deutschen und europäischen urologischen Fachgesellschaften zusammengefasst dargestellt (Leitlinie Prostatakarzinom 2019; Mottet et al. 2017):

\section{Niedriges Risiko nach D’Amico:}

Innerhalb von zehn bis fünfzehn Jahren ist die prostatakarzinomspezifische Mortalität in dieser Gruppe gering. Eine kurative Therapie kann den Patienten bei einer Lebenserwartung von über zehn Jahren empfohlen werden.

Für Patienten mit einer Lebenserwartung über zehn Jahre sind kurative Behandlungsoptionen eine radikale Prostatektomie oder kurative Bestrahlungstechniken (Wilt et al. 2012).

Die radikale Prostatektomie konnte als einziges Therapieverfahren einen Überlebensvorteil im Vergleich zur konservativen Therapie in randomisierten Studien belegen (Bill-Axelson et al. 2008; Bill-Axelson et al. 2011).

Eine aktive Überwachung kann für ausgewählte Patienten empfohlen werden. Fokale Therapien (Hochintensiver fokussierter Ultraschall (HIFU), irreversible Elektroporation (IRE) etc.) sind gegenwärtig noch als experimentell anzusehen (Ganzer et al. 2017).

Für Patienten mit einer Lebenserwartung unter zehn Jahren ist eine Watchful-Waiting- Strategie die Maßnahme der Wahl. Patienten im Progress der Erkrankung können dann entweder nachfolgend noch eine kurative Therapie erhalten oder es erfolgt ein Verzicht auf jegliche kurative Therapie und es wird eine Hormontherapie im Metastasierungsstadium als palliatives Therapiekonzept begonnen.

\section{Mittleres und hohes Risiko nach D'Amico:}

Bei diesen Patienten besteht ein relevantes Risiko, innerhalb von zehn Jahren ein Fortschreiten der Erkrankung zu erleiden oder innerhalb von 15 Jahren durch das Prostatakarzinom zu versterben. Eine kurative Therapie der Patienten sollte ab einer Lebenserwartung von fünf bis zehn Jahren erfolgen.

Für Patienten mit einer Lebenserwartung von über zehn Jahren ist die radikale Prostatektomie das Mittel der Wahl. Vor allem Patienten mit einem mittleren bzw. hohen Risiko nach D'Amico profitieren, wie in retrospektiven Studien belegt, von einer radikalen Prostatektomie (Boorjian et al. 2012). 
Bei einer R1-Resektion sollte gegebenenfalls eine adjuvante Strahlentherapie, bei N1 gegebenenfalls eine adjuvante Hormontherapie erfolgen. Eine Alternative stellt die kurative Strahlentherapie mit adjuvanter Hormontherapie dar.

Für Patienten mit einer Lebenserwartung unter zehn Jahren sollte bei subvesikaler Obstruktion die radikale Prostatektomie empfohlen werden. Andernfalls stehen die kurative Bestrahlung und adjuvante Hormontherapie zur Verfügung.

Für Patienten mit einer Lebenserwartung unter fünf Jahren kann bei einer subvesikalen Obstruktion eine transurethrale Resektion der Prostata (TUR-P) erfolgen. Eine kurative Therapie ist hier nicht zu empfehlen. Eine Hormontherapie ist ohne Hinweis auf Metastasen nicht indiziert.

\subsection{Radikale Prostatektomie}

Für das Prostatakarzinom und dessen Prognose haben sich der Gleason-Score, die TNM (Tumor, Nodus und Metastasen, Tabelle 1) -Kategorie sowie der Resektions-Status des Karzinoms nach Operation als primär klinisch relevant erwiesen.

Die radikale Prostatektomie besteht aus der operativen Entfernung der gesamten Prostata und zusätzlich der Samenblasen beidseits, des distalen Anteils der Samenleiter beidseits und der prostatischen Harnröhre. Die einzelnen Schritte der Operation werden in 1.3 für das Da-Vinci-Operationsverfahren beschrieben.

Das Operationsverfahren wurde erstmals im Jahre 1945 von Millin (1945) aus dem All Saint Hospital London beschrieben. Von der Vorgehensweise berichtete P. C. Walsh 1982 aus dem James Buchanan Brady Urological Institute in der Johns Hopkins Medical School (Walsh und Donker 1982; Walsh et al. 1983).

Heute sind verschiedene Operationstechniken sowie Zugangswege für die radikale Prostatovesikulektomie üblich. Die heutigen operativen Zugänge sind retropubisch, perineal, laparoskopisch (intra- und extraperitoneal) oder roboterassistiert (intra- und extraperitoneal).

Der intraoperative Blutverlust und die Transfusionsraten sind beim laparoskopischen (einschließlich roboterassistierten) Vorgehen geringer. Gegenüber der offenen retropubischen Operation sind potentielle Vorteile: Geringerer perioperativer Schmerzmittelbedarf, kürzere Katheterverweildauer, bessere postoperative Erholung, kürzere Fehlzeiten an der Arbeitsstelle und kürzerer Krankenhausaufenthalt. Nachteilig könnten eine längere Operationszeit sowie eine längere Lernkurve sein (Tooher et al. 2006). Eine Studie zeigte schlechtere Ergebnisse in Bezug auf das Wiedererlangen der Potenz nach laparoskopischer gegenüber der roboterassistierten radikalen Prostatektomie (Asimakopoulos et al. 2011).

Es existieren retrospektive Untersuchungen zur perioperativen Morbidität und Mortalität in Bezug auf die Zahl der RPE pro Operateur und Krankenhaus. Kurz- und langfristige Komplikationen sind geringer, wenn die Operation in Zentren mit hohem Aufkommen 
und entsprechend erfahrenen Operateuren durchgeführt wird (Barocas et al. 2010; Loeb et al. 2008; Nuttall et al. 2004). Die deutsche Krebsgesellschaft verlangt aufgrund dessen für die Zertifizierung von Prostatazentren eine Mindestzahl von 100 durchgeführten Operationen pro Operateur und 25 Operationen pro Jahr (Leitlinie-Prostatakarzinom 2019).

\subsection{Da-Vinci-Operationsverfahren für die radikale Prostatektomie}

Eine der ersten Beschreibungen des Da-Vinci-Operationsverfahrens wurde 2001 publiziert (Abbou et al. 2001; Binder und Kramer 2001). Im weiteren Verlauf etablierte sich diese Operationstechnik weltweit. Nachfolgend wird kurz das Da-Vinci-Operationssystem beschrieben.

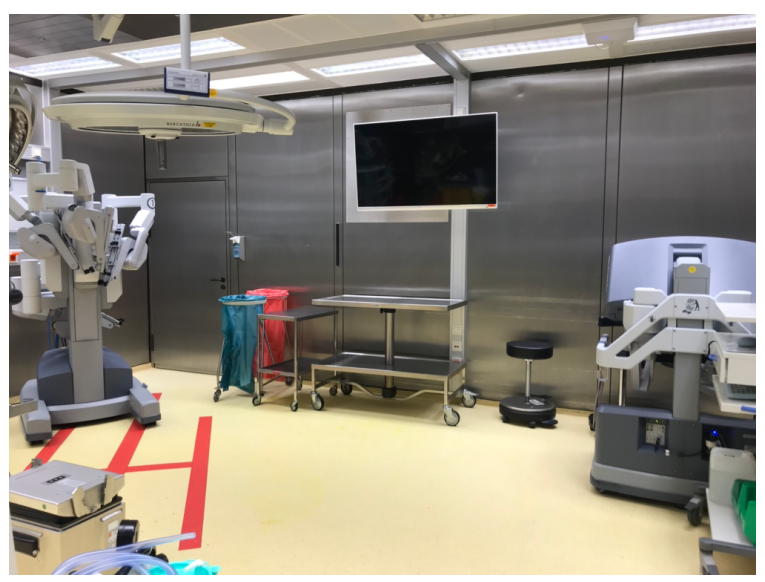

Abbildung 2: Operationssaal mit Da-Vinci-Operationssystem.

Das Da-Vinci Operationssystem ermöglicht dem Operateur durch kleine Inzisionen komplexe Operationen durchzuführen. Das Operationssystem besteht aus drei verschiedenen Komponenten (Abbildung 2). Benötigt wird unter anderem eine Konsole (Abbildung 3), welche dem Operateur eine ergonomische Steuerung des Systems während der Operation ermöglicht. Eine weitere Komponente ist der Patientenwagen (Abbildung 4), welcher während der Operation für die Steuerung der Instrumente sowie der Kamerasysteme zuständig ist. 


\subsubsection{Operateurskonsole}

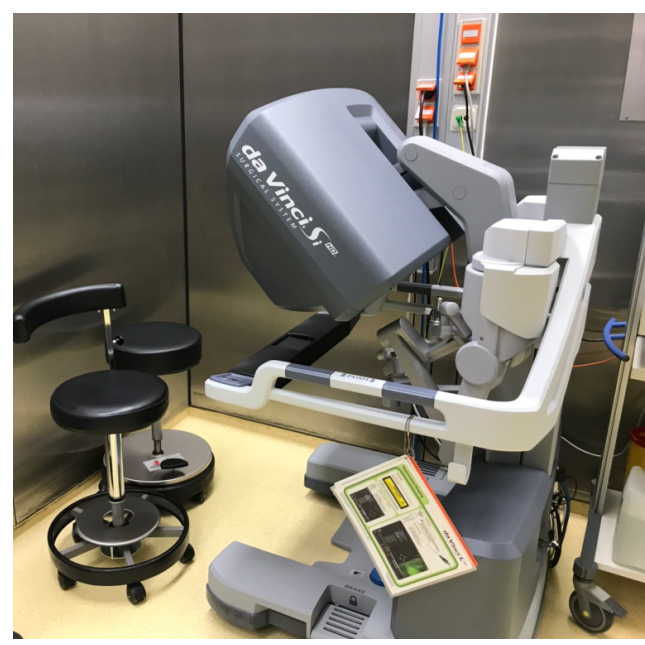

Abbildung 3: Operateurskonsole des Da-Vinci-Operationssystems.

Der Operateur sitzt während der Operation komfortabel an der Konsole und kann mittels einer 3-D-Optik die Operation am Patienten durchführen. Die Hände des Operateurs greifen in die Master Controller unterhalb des Displays und führen die Instrumente während der Operation. Das System überträgt die Finger-, Handgelenks- sowie die Armbewegung des Operateurs während der Operation in Echtzeit und in präzisen Bewegungen auf die Instrumente.

\subsubsection{Patientenwagen}

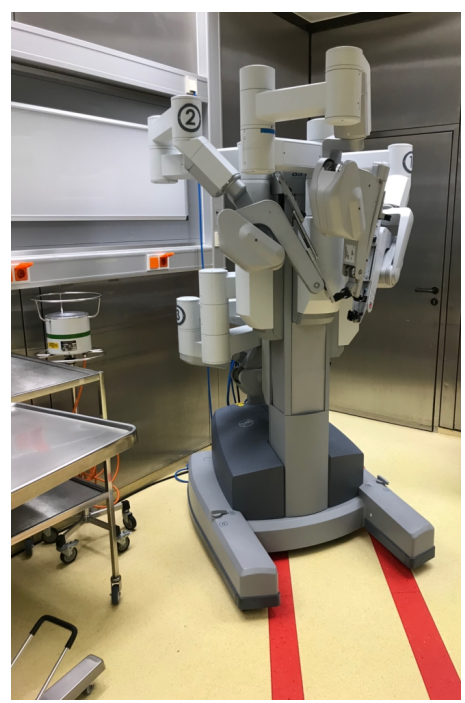

Abbildung 4: Personenwagen des Da-Vinci-Operationssystems.

Dieser Teil des Systems befindet sich während der Operation am Patienten und steuert durch die Kommandos des Operateurs die Instrumente im Patienten. Es sind drei bis vier robotische Arme angedockt. Die Arme bewegen sich an der Drehpunktbefestigung. Das 
System sorgt dafür, dass die Instrumente nur vom Operateur gesteuert werden können. Sicherheitssysteme sorgen dafür, dass keine eigenständigen Bewegungen möglich sind.

\subsubsection{Instrumente}

Es gibt ein breites Spektrum an Instrumenten für die Operation (Nadelhalter, Scheren, etc.). Die Instrumente verfügen über sieben verschiedene Bewegungsgrade. Somit werden selbst die Bewegungsgrade der menschlichen Hand übertroffen.

Die einzelnen OP-Schritte, wie sie an unserer Klinik erfolgen, werden zusammenfassend nachfolgend beschrieben:

Die Operation wird in Rückenlage des Patienten durchgeführt. Beginnend werden die intraabdominellen Zugangswege für die Trokare etabliert. Es werden in der Regel ein Kameratrokar und eine variable Anzahl von verschiedenen weiteren vier bis fünf Trokaren verwendet. Im ersten operativen Schritt wird die Harnblase von der Bauchdecke abpräpariert. Im weiteren Verlauf wird die Prostata exponiert. Die Beckenbodenfaszie wird seitlich eröffnet. Weiter folgt die Präparation des Apexbereichs. Anschließend wird der Blasenhals eingeschnitten, abgesetzt und in die Tiefe zu den Samenblasen präpariert. Die Gefäßnervenbündel der Prostata können je nach onkologischer Situation des Patienten geschont werden. Dies erfolgt mit dem Ziel, die Erektionsfähigkeit des Patienten zu erhalten. Dabei muss eine Schnellschnittuntersuchung der Prostata erfolgen, um die Tumorfreiheit zu gewährleisten. Das Präparat wird mittels Bergebeutels während der Operation geborgen und in die Pathologie gesendet. Sollte Tumorkontakt zum Schnellschnitt bestehen, muss das Gefäßnervenbündel nachreseziert werden. Ergänzend erfolgt eine obturatorische Lymphadenektomie beidseits zur Bestimmung des lokalen N-Stadiums des Prostatakarzinoms. Anschließend erfolgt die Anastomose zwischen Harnröhre und Harnblase. Hiernach wird für ca. sieben Tage ein transurethraler Dauerkatheter zur Gewährleistung der Heilung und eines kontinuierlichen Urinabflusses eingelegt. Abschließend erfolgt die Einlage einer Wunddrainage, das Abdocken des Systems und die Naht der kleinen Hautschnitte mittels Intrakutannaht und Verschluss mit einem Hautkleber.

Die einzelnen operativen Schritte werden durch den Operateur mittels der Da-VinciInstrumente durchgeführt und es erfolgt eine laparoskopische Assistenz durch einen ärztlichen Kollegen steril am Tisch. Je nach Befund (Vor-Operationen, körperliche Konstitution des Patienten, Nerverhalt mit Schnellschnitt) umfasst die Operation eine Operationsdauer von zwei bis fünf Stunden. Ziel der Operation ist stets eine onkologisch kurative R0Situation sowie ein gutes funktionelles Ergebnis zu schaffen (Kontinenz, Potenz) (Asimakopoulos et al. 2011; Bott et al. 2003; Eastham 2008; Ruurda et al. 2002; Trinh et al. 2012; Walsh et al. 1983). 


\subsection{Lärm und Musik im Operationssaal}

Der Einfluss von Lärm auf die kardiovaskuläre Gesundheit wurde in mehreren Studien untersucht und ein direkter Zusammenhang nachgewiesen. Zum einen zeigt eine Exposition von Straßenlärm (60 Dezibel $(\mathrm{dB})$ ) einen direkten Zusammenhang mit einem erhöhten Risiko für Bluthochdruck (Babisch 2000; Herbold et al. 1989). Weitere Studien offenbarten zum anderen, dass es auch einen Zusammenhang zwischen Flugzeuglärm und kognitiver Entwicklung von Kindern gibt (Stansfeld et al. 2005). Es sind Hinweise vorhanden, dass Lärm einen Einfluss auf die Entwicklung von schwerwiegenden Erkrankungen wie Osteoporose, koronare Herzerkrankung, Diabetes mellitus und Bluthochdruck durch einen erhöhten Kortisonspiegel hat (Kirschbaum und Hellhammer 1999; McEwen 1998).

Der Einfluss von Lärm im Operationssaal war bereits Thema verschiedener Studien. Es zeigen sich unterschiedliche Effekte auf das Ergebnis der Operation, die Leistung des Operateurs sowie auf die Gesundheit bzw. kardiovaskulären Risikofaktoren der Operateure (wie arterieller Hypertonus oder KHK-Risiko) (Allen und Blascovich 1994; Aydin und Kaltenbach 2007; Conrad et al. 2010; Conrad et al. 2012; Kirschbaum und Hellhammer 1999; Lee et al. 2013; MacClelland 1979; Moorthy et al. 2004; Sevdalis et al. 2007; Shapiro und Berland 1972).

Im Operationssaal können sehr hohe Lärmpegel registriert werden. Diese liegen bei verschiedenen Messungen in den Studien zwischen 80 und 85 dB. Frühe Studien wiesen einen Anstieg der Lautstärke durch die Nutzung von Instrumenten oder durch Fallenlassen von Materialen mit Lautstärkespitzen von bis zu 108 dB nach (Hodge und Thompson 1990). Für eine normale Arbeitsumgebung wird ein Lärmpegel von $45 \mathrm{~dB}$ empfohlen (Cabrera und Lee 2000; Conrad et al. 2010; Falk und Woods 1973; Shapiro und Berland 1972). Somit stellen die gemessenen Lautstärken einen deutlichen Stressfaktor für das ganze Operationsteam und vor allem den Operateur dar (Conrad et al. 2010). Eine weitere Studie wies ein höheres Stresslevel bei Operationen mittels Laparoskopie im Gegensatz zu offen durchgeführten Operationen nach (Berguer et al. 2001). Weitere Untersuchungen verwiesen auf eine erhöhte Fehlerquote des Operateurs bei chirurgischen Aufgaben durch Lärmbelastung (Moorthy et al. 2003). Es wird vermutet, dass der erhöhte Lärmpegel zu einer Dilatation der Pupillen und zu einer peripheren Vasokonstriktion als Stresszeichen bei Operateuren führt (Shapiro und Berland 1972). Der mögliche Effekt von Lärm im Operationssaal hängt unter anderem auch von der Erfahrung des Operateurs ab. Unerfahrene Operateure sind vermeintlich lärmempfindlicher als erfahrene Operateure (Moorthy et al. 2004; Reznick 1993). Im Gegensatz dazu zeigte eine weitere Studie, dass Lärm als unabhängiger Stressfaktor keinen Einfluss auf die technische Leistung laparoskopischer oder offener Operationstechniken hat (Moorthy et al. 2003). Somit bleibt der tatsächliche Einfluss von Lärm während einer Operation unklar, wie eine Meta-Analyse offenlegte (Arora et al. 2010). 
Ein weiterer Gesichtspunkt im Rahmen vergleichender Untersuchungen ist die Verwendung von Musik im Operationssaal. Untersuchungen haben beschrieben, dass Musik im Operationssaal den Patienten beruhigt und von der Prozedur ablenkt. Musik schafft eine wärmere und angenehmere Umgebung für Patienten und das Personal (MacClelland 1979). Moris und Linos (2013) wiesen einen Effekt von Musik auf das Stressempfinden des Patienten und dessen Narkosebedarf während der Operation in einer Meta-Analyse von 24 Arbeiten nach. Eine weitere Arbeit hat die Wirkung von Musik auf die Leistungsfähigkeit der Operateure während roboterassistierter laparoskopischer Operationsübungen, wie z. B. Nähübungen am Holzphantom, untersucht. Siu et al. (2010) konnten eine signifikante Optimierung für bestimmte Operationsschritte, für die zurückgelegte Distanz, die Verminderung der Muskelaktivität und eine Verminderung der Frequenz der Muskelaktivitäten zeigen.

Aktuell gibt es keine wissenschaftliche Arbeit, welche die Lärmbelastung im Operationssaal sowie die Anwendung von Musik bei Operationen im besonderen Umfeld des roboterassistierten Da-Vinci-Verfahrens bei der radikalen Prostatektomie untersucht. Diese Arbeit fokussiert sich somit auf diese besondere Kommunikationssituation und die operativen Herausforderungen des Da-Vinci-Systems im Hinblick auf die damit verbundene Lärmbelastung und Kommunikationsprobleme im Operationsablauf.

\subsection{Das SOTOS}

Das „Silent Operating Theatre Optimisation System“ (SOTOS) ist ein Kommunikationstool für den Einsatz im Operationssaal (Abbildung 5).

Das System wurde von Herrn PD Dr. med. Martin Friedrich (Klinik für Thorax-, Herzund Gefäßchirurgie, Universitätsmedizin Göttingen) entwickelt und patentiert (Friedrich et al. 2017). Im Auszug der Patentschrift wird das System wie folgt beschrieben (Patentnummer: DE102015205463A1):

„Eine Vorrichtung zur Bildung von Audioumgebungen in einem Operationsraum wird beschrieben. Die Vorrichtung umfasst eine Vielzahl an tragbaren Gegensprecheinheiten. Die Gegensprecheinheiten weisen jeweils mindestens ein Mikrofon und einen Kopfhörer auf, welche zur Unterdrückung von Umgebungsgeräuschen ausgebildet sind. Ein Mischer steht mit der Vielzahl an Gegensprecheinheiten in Signalaustausch und ist zur programmgesteuerten Signalleitung zwischen der Vielzahl an Gegensprecheinheiten ausgebildet. In einem Speicher sind unterschiedliche Steuerprogramme, die jeweils die programmgesteuerte Signalleitung festlegen, gespeichert. Mindestens eines der Steuerprogramme schränkt den Signalaustausch zwischen der Vielzahl an Gegensprecheinheiten zur Bildung der Audioumgebungen ein. Eine Steuerung bestimmt eines der Steuerprogramme und steuert den Mischer zur programmgesteuerten Signalleitung zwischen der Vielzahl an Gegensprecheinheiten gemäß dem bestimmten Steuerprogramm.“ 

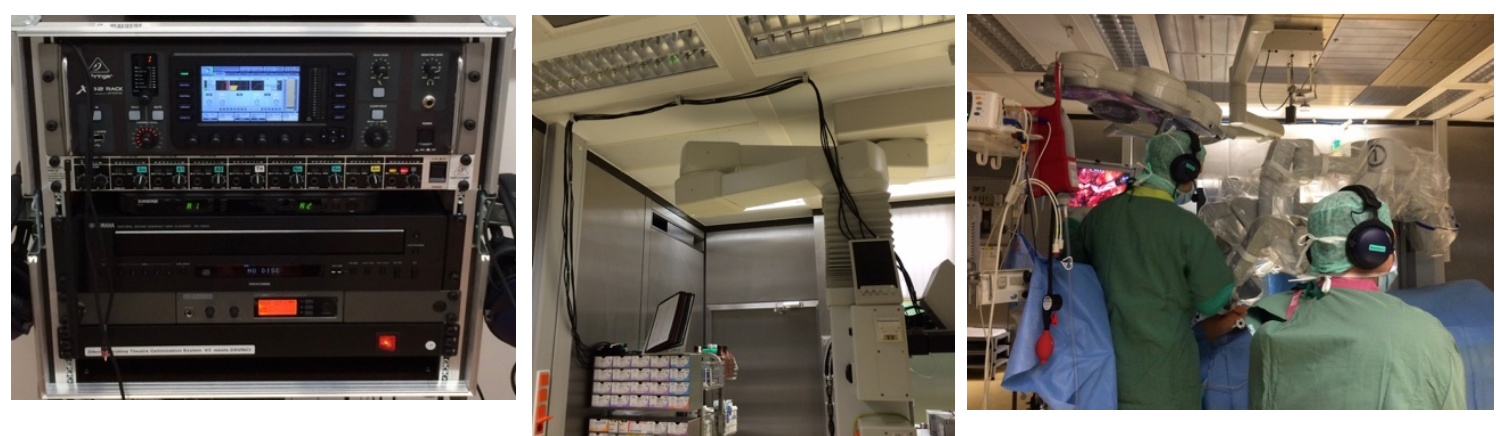

Abbildung 5: SOTOS in der Verwendung im Operationssaal (von links: Steuereinheit, Verkabelung im Operationssaal und Personal mit Verwendung der Kopfhörer/Mikrofonkombination).

Dieses System ist in praxi mehrfach getestet und optimiert worden. SOTOS wurde dann in der alltäglichen chirurgischen Arbeit der Klinik für Urologie im Rahmen der roboterassistierten radikalen Prostatektomie erprobt (Abbildung 5). Der erste Einsatz des Systems erfolgte Anfang des Jahres 2016. Im Weiteren erfolgte die Optimierung der Einstellungen des Systems für die Anforderungen des Da-Vinci-Operationssystems.

Es wurden das Operationsteam (bestehend aus Operateur, Assistent(en) und OPFachpersonal und das Anästhesieteam mit dem System (bestehend aus jeweils einem Headset mit Kopfhörern und einem Mikrofon) ausgestattet, um die Kommunikation innerhalb des Gesamtteams zu verbessern. Alle Teilnehmer sind während der gesamten Operation mit dem SOTOS verbunden und können miteinander kommunizieren. Die komplette Verarbeitung der Signale der kabelgebundenen Teilnehmer sowie der Teilnehmer mit Funkheadset erfolgt über eine zentrale Steuereinrichtung. Diese verarbeitet die Signale und steuert die Unterdrückung der eingespielten Musik, wenn ein Teilnehmer in sein Mikrofon spricht. Durch den technischen Einsatz dieser Steuereinrichtung erfolgt eine sanfte Steuerung der Gespräche und der Musik durch Filterung von zu lauten Geräuschen und leisen Nebengeräuschen durch bestimmte Beschränkungen der Lautstärke.

Die Installation der Verkabelung erfolgt im Operationsaal parallel zu den Installationen für das Da-Vinci-Operationssystem. Die Verkabelung stört den Ablauf der Operation nicht und kommt nicht mit dem sterilen OP-Feld in Berührung.

\subsection{Fragestellung}

Diese Dissertation beschäftigt sich mit der Lärmbelastung und dem Einsatz von Musik im Operationssaal und deren Einfluss auf die Operation, das Stressempfinden des Operateurs und des gesamten Teams. Ziel dieser Arbeit ist es, in einem prospektiven Ansatz den Einsatz des SOTOS bei Operationen in der Urologie am Beispiel der radikalen laparoskopischen roboterassistierten Prostatovesikulektomie mit dem Da-Vinci-Operationssystem zu 
evaluieren. Untersucht werden soll die Auswirkung auf das Operationsergebnis und ob der Einsatz dieses Systems zu einer Verbesserung für das Operationsteam im Hinblick auf die Stress- und Lärmbelastung führt. 


\section{$2 \quad$ Material und Methodik}

Ziel der Studie war die Evaluierung einer Stresssenkung und Lautstärkenreduktion während der radikalen laparoskopischen roboterassistierten Prostatovesikulektomie durch den Einsatz des SOTOS. Im Folgenden werden die Studienpopulation vorgestellt sowie der Ablauf der Messungen während der Operation und die Methoden zur Stress- und Lautstärkemessung beschrieben.

\subsection{Versuchsaufbau des SOTOS}

Der Aufbau des SOTOS erfolgte bei jeder durchgeführten Messung gleich. Als Grundlage diente der vorgegebene und standardisierte Aufbau des Operationssaales während einer daVinci-Operation (Abbildung 6).

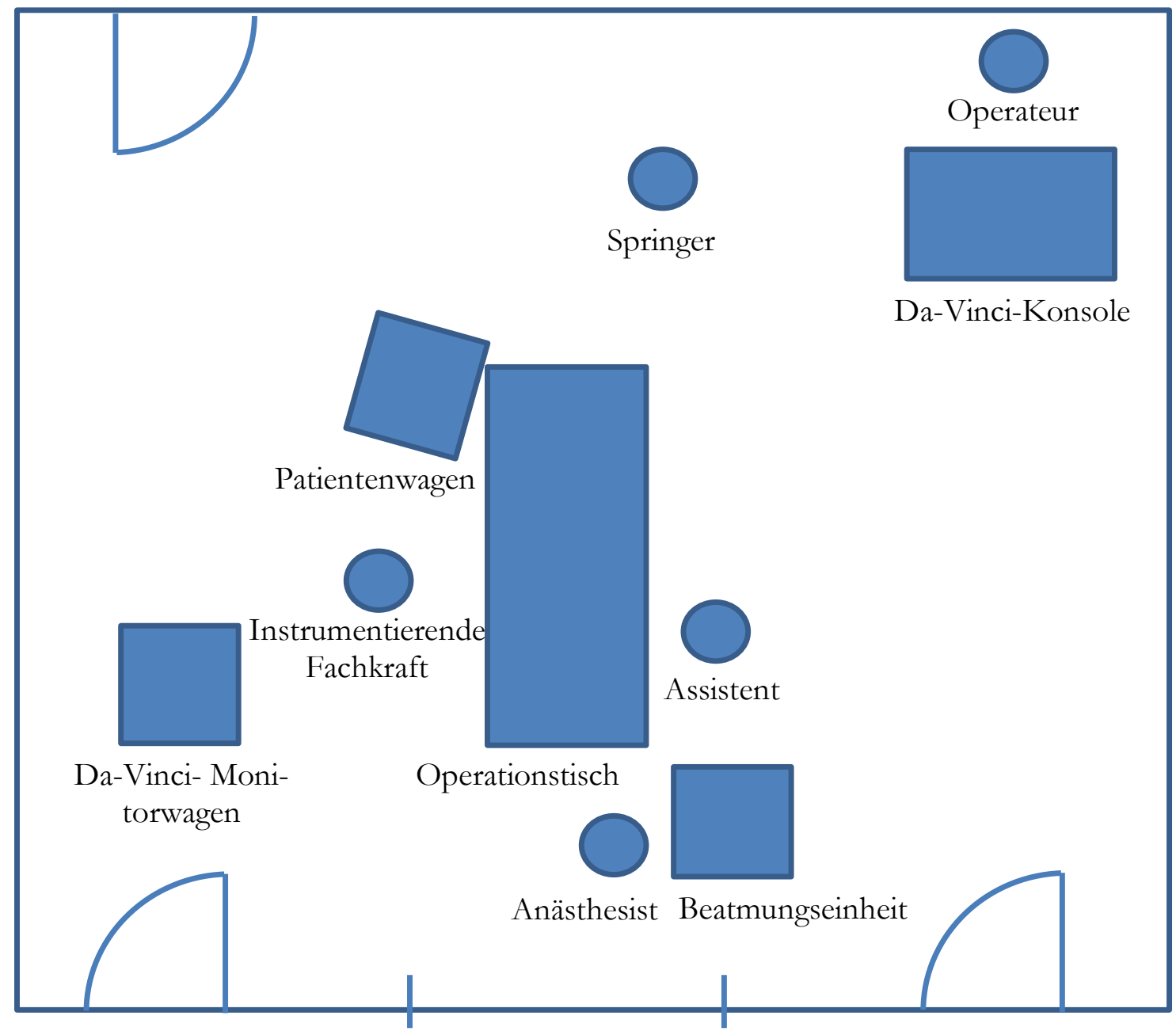

Abbildung 6: Aufbau des Da-Vinci-Operationssaales.

Der Einsatz des Da-Vinci-Systems bedingte eine feste Verteilung der einzelnen Positionen im Operationssaal. Mittig im Operationssaal befand sich der Operationstisch. Auf diesem lag der zu operierende Patient. Am Kopfende des Operationstisches befand sich die Beatmungseinheit, von dieser aus der Anästhesist den Patienten während der kompletten Ope- 
ration betreute. Direkt steril am OP-Tisch arbeitete die instrumentierende Fachkraft, welche die Instrumente anreichte, und der Assistent, der die Operation mit betreute. Zudem befand sich dort der Patientenwagen mit den steril abgedeckten Operationsarmen. Am Rande des Operationssaales war die Konsole, von der aus der Operateur die Instrumente steuerte, positioniert. Frei im Operationssaal bewegte sich der Springer und hatte somit keine feste Position während der Operation. Ein komplettes OP-Team bestand somit also aus Operateur, Assistent, Anästhesist, instrumentierende Fachkraft und Springer. Aufbau und Aufgabenverteilung im Operationssaal waren während der 32 durchgeführten Messungen in beiden Studienarmen identisch.

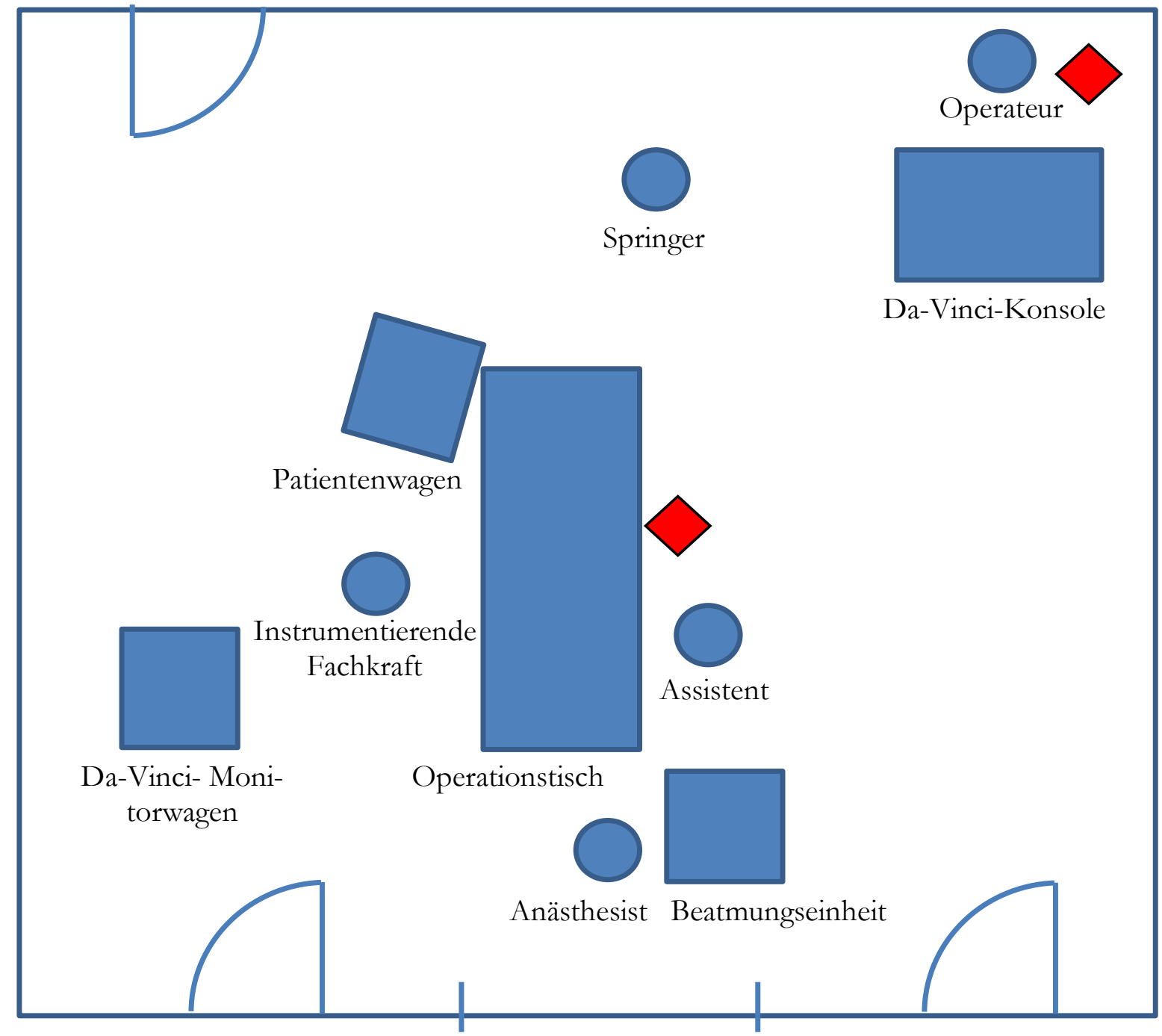

Abbildung 7: Standort der Lautstärkemessgeräte (rote Raute) zur Messung des Schalldruckpegels (jeweils $1 \mathrm{~m}$ Abstand zur Konsole bzw. zum Operationstisch).

Speziell zur Durchführung der Messungen fügten wir einige technische Geräte und Messaufbauten dem Operationssaal hinzu. Das OP-Personal haben wir in das System und seine verschiedenen Funktionen eingewiesen, um während der Untersuchungen einen reibungslosen Ablauf zu gewährleisten. 
Wir installierten insgesamt zwei Lautstärkemessgeräte in jeweils einem Meter Abstand zur Konsole sowie zum Operationstisch (Abbildung 7).

Über diese Messgeräte wurden während der Operation der Schalldruckpegel in Dezibel mit der Wichtung A (db(A)) jede Sekunde gemessen.

Wurde SOTOS verwendet, erfolgte zusätzlich der Aufbau der SOTOS-Einheit. Das OPTeam wurde mit funk- oder kabelgebundenen Kopfhörern und Mikrofonen ausgestattet (Abbildung 8).

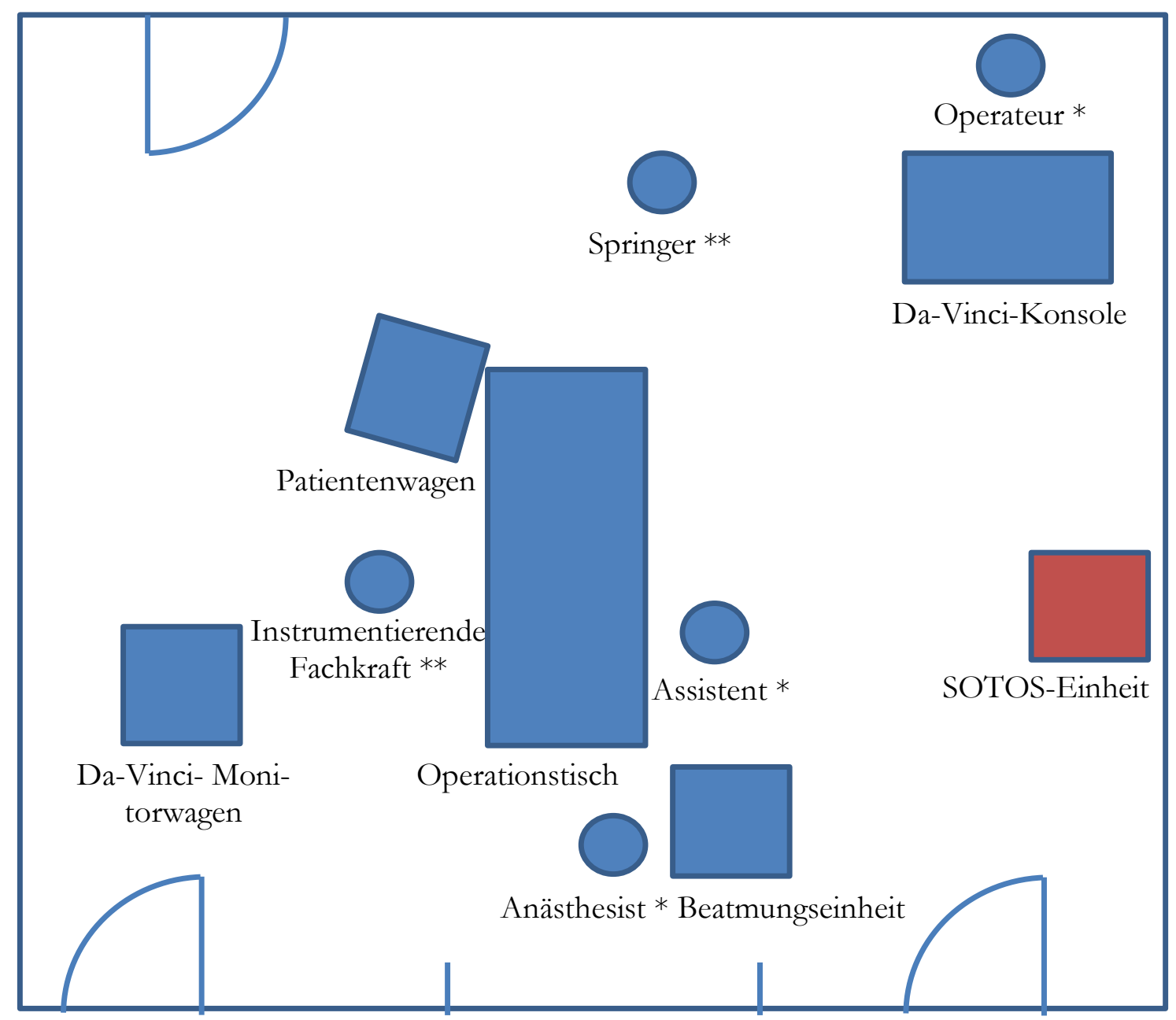

Abbildung 8: Messaufbau während eines Messtages mit Einsatz des SOTOS (rot, $n=16$; *kabelgebundene Kopfhörer/Mikrofone; ** funkgebundenene Kopfhörer/Mikrofone). 
Die instrumentierende Fachkraft und der Springer waren mit Funkkopfhörern sowie Funkmikrofonen ausgestattet (Abbildung 9). So war deren freie Beweglichkeit während der Operation gewährleistet.

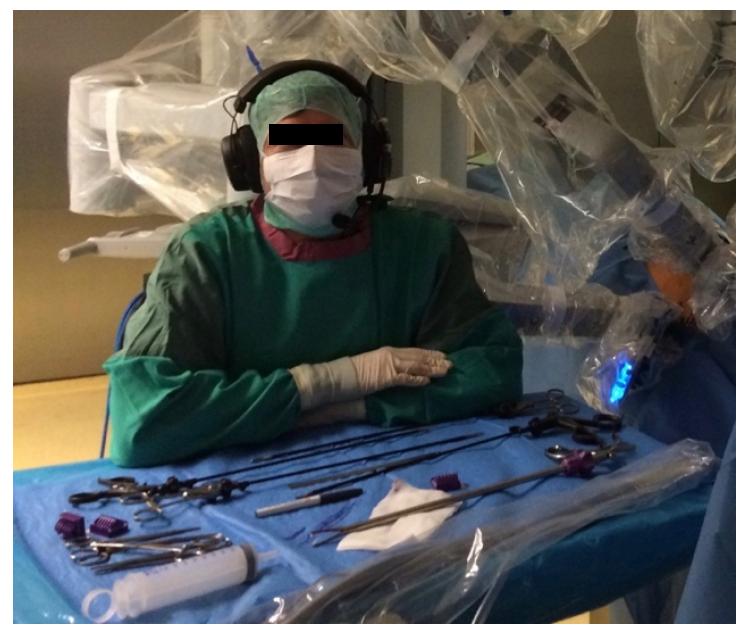

Abbildung 9: Instrumentierende Fachkraft trägt eine Funkkombination.

Der Operateur, der Assistent und der Anästhesist wurden hingegen mit kabelgebundenen Kopfhörern mit integrierten Mikrofonen ausgestattet (Abbildung 10).

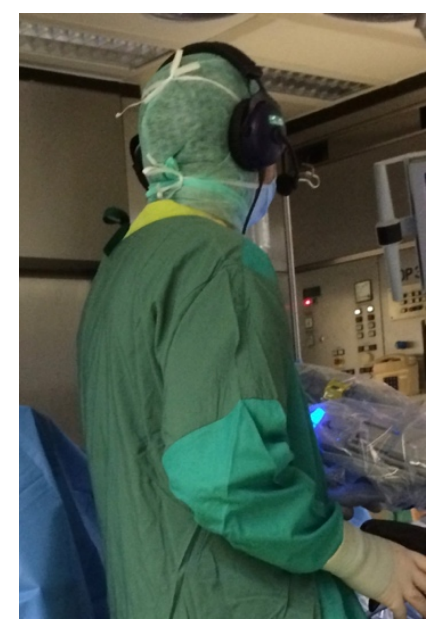

Abbildung 10: OP-Assistent trägt eine kabelgebundene Kommunikations-Kombination.

Alle Teilnehmer waren über eine zentrale Steuereinrichtung (Abbildung 11) miteinander verbunden und konnten so störungsfrei miteinander kommunizieren. 


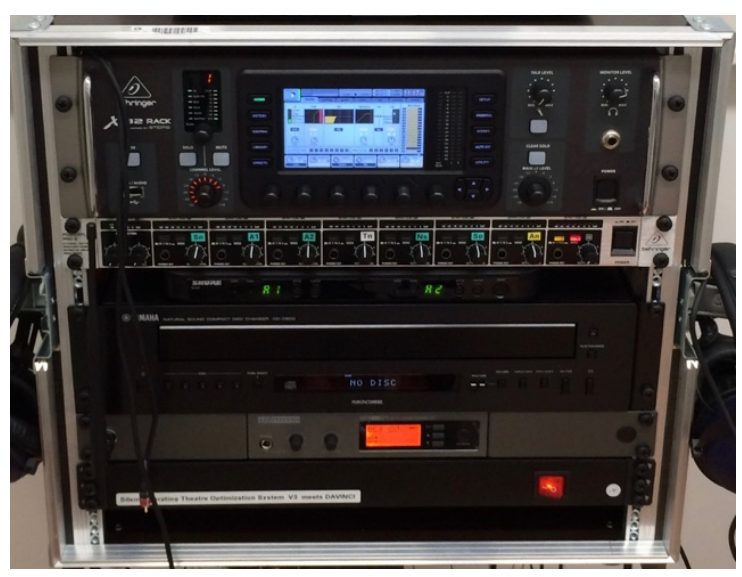

Abbildung 11: Zentrale Steuereinheit des SOTOS.

Zudem wurde über das System Musik eingespielt, die während der gesamten Operation kontinuierlich wiedergegeben werden konnte. Während jeglicher Kommunikation wurde jedoch die Musik sofort unterbrochen bzw. in den Hintergrund gespielt. Sobald ein Teilnehmer zu sprechen begann, reduzierte die zentrale Steuereinrichtung die Musiklautstärke und nach Beendigung des Gespräches steigerte sich die Lautstärke auf das vorherige Niveau. Während der kompletten Operation war somit eine ungestörte Kommunikation zwischen den Teilnehmern möglich. Eine Mischung aus POP- und Rock-Künstlern diente als Grundlage für die Musikauswahl. Diese wurde im Vorfeld mit dem Operationsteam abgestimmt, sodass keine grundlegende Abneigung gegen die Musikauswahl bestand.

\subsection{Studienpopulation}

Alle Patienten erhielten eine radikale roboterassistierte laparoskopische Prostatovesikulektomie mit systematischer pelviner Lymphadenektomie beidseits. Die Operationen wurden entweder mit oder ohne Schnellschnittuntersuchung bzw. Erhalt des Gefäßnervenbündels durchgeführt.

Wir stellten die Studie prospektiv für insgesamt 32 Operationen (16 Messungen in jedem Studienarm) auf. Die Operationen erfolgten im Zeitraum von März bis Juli 2017. 16 Operationen erfolgten unter Verwendung des SOTOS und 16 ohne die Verwendung des Systems. Die Messungen hatten keinen Einfluss auf den Ablauf der Operation an sich. Die Operationsschritte wurden in der SOTOS- sowie in der Kontrollgruppe in üblicher Technik gleich durchgeführt (siehe 1.3.) (Ruurda et al. 2002). Wir entschieden uns für eine begrenzte Anzahl an Operationen, um die organisatorische und logistische Durchführung der Messtage fortlaufend mit standardisierten Messbedingungen zu gewährleisten.

Im Vorfeld war den Teilnehmern, bestehend aus dem Operateur, dem Assistenten, dem Anästhesisten, der instrumentierenden Fachkraft sowie dem Springer, nicht bekannt, ob es sich an dem Operationstag um eine Messung in der SOTOS- oder in der Kontrollgruppe handelte. Präoperativ erfolgte deshalb eine Randomisierung mittels einer im Vorfeld durch 
Zufall erstellten Randomisierungsliste. Falls aus organisatorischen Gründen oder durch den Operationsablauf bedingt die Messung abgebrochen werden musste, wurde diese nicht gewertet und eine neue Messung zu einem späteren Zeitpunkt nach Randomisierung nachgeholt.

Die persönlichen Daten der Teilnehmer sowie der Patienten wurden anonymisiert und gingen nicht in die Studie ein. Vor dem Beginn wurde die Studie in der Ethikkommission der Universitätsmedizin Göttingen besprochen und eine Freigabe mittels Ethikvotum (DOK_204_2015) erteilt.

\subsection{Erfassung der klinischen Daten der jeweiligen Operationen}

Die Erfassung der klinischen Parameter während der Operation und im Verlauf geschah anhand der Operationsberichte, Ambulanz-Berichte sowie Arztbriefe. Dies erfolgte anonymisiert und ohne Bezug zu den jeweiligen Patienten. Wir erfassten die Daten zur Operation wie abdominelle Voroperationen, Operationsdauer und schwierige OperationsVerhältnisse bzw. Komplikationen. Komplizierte Operations-Faktoren und/oder Komplikationen dokumentierte der Operateur standardmäßig im OP-Bericht. Intraoperative Komplikationen waren als erhöhter Blutverlust (über $300 \mathrm{ml}$ ) oder etwaige Verletzungen des Dünn- bzw. Dickdarms insbesondere des Rektums, der Gefäße oder der Nerven definiert. Schwierige Operationsverhältnisse waren durch abdominelle Voroperationen oder Verwachsungen im Situs dokumentiert worden. Während der Operation erfolgte bei manchen Patienten eine intraoperative Untersuchung der entnommenen Prostata mittels mikroskopischer Begutachtung durch den Pathologen (Schnellschnitt). Hier evaluierte der Pathologe, ob die Schnittränder am Rand der Prostata hin zum Gefäßnervenbündel tumorfrei waren. Dieses Verfahren definierten wir als zusätzlichen Aufwand für die Operation aufgrund schwierigerer Präparation des OP-Situs und der Wartezeit auf den Schnellschnitt (Asimakopoulos et al. 2011).

Zusätzlich wurden Daten zur Tumorformel und zum Patientenalter erhoben. Wir erfassten das initiale Tumorstadium (Gleason-Score, initialer Serum-PSA-Wert bei Diagnose (iPSA), körperliche Untersuchung, insbesondere Tastbefund der Prostata und Klassifizierung nach D'Amico et al. (2006) sowie das postoperative Tumorstadium (pTNM und Gleason-Score (Svanholm und Mygind 1985)). Auch wurden postoperative Komplikationen wie Nachblutung, Anastomoseninsuffizienz oder Wundheilungsstörungen erfasst. Gesondert registrierten wir Lymphozelen und postoperative Infektionen wie Harnwegsinfekte oder Wundinfektionen. Es erfolgte auch die Dokumentation der Dauer des Krankenhausaufenthaltes der Patienten. 


\subsection{Durchführung eines Messtages}

Vor der Durchführung des Studientages erfolgte eine Überprüfung der Ausschlusskriterien für die Messungen. Die Ausschlusskriterien waren eine Besiedlung des Patienten mit einem multiresistenten Keim (wie Methicillin-resistenter Staphylococcus aureus (MRSA), Vancomycin-resistente Enterokokken (VRE) und multiresistente gramnegative Bakterien (MRGN)), die Operation wurde geplant von mehr als einem hauptverantwortlichen Operateur durchgeführt, die technische Auswertung der EKG-Messungen war nicht gewährleistet oder es handelte sich um eine geplante offene radikale Prostatovesikulektomie. Falls eines dieser Kriterien zutraf, resultierte ein Ausschluss aus der Studie.

Im Rahmen der im Vorfeld per Zufall erstellten Randomisierungsliste erfolgte eine Einteilung der Messung in die SOTOS- oder Kontrollgruppe. Vor Beginn der Operation wurde die Technik, insbesondere die Lautstärkemessung, installiert und auf ihre Funktionalität überprüft (Aufbau siehe 2.1. Versuchsaufbau des SOTOS).

Die Bewertung der Stressbelastung während einer Operation erfolgte anhand von EKGMessungen. Alle Beteiligten des OP-Teams (Operateur, Assistent, Anästhesist, instrumentierende Fachkraft und Springer) erhielten ein Langzeit-EKG-Messgerät zur Protokollierung der Herzfrequenz während der Operation. Der genaue Ablauf wird im Kapitel 2.5. EKG-Messungen beschrieben.

Im Rahmen der Studie integrierte das Institut für Sozial- und Kommunikationspsychologie außerdem einen Konzentrationstest. Für den Konzentrationstest wurde die Konzentrations-Leistungs-Test-Revidierte Fassung (KLT-R) verwendet (Mayrhofer 2001). Alle Beteiligten des OP-Teams absolvierten diesen schriftlichen Test vor und nach der Operation. Die Veröffentlichung der Ergebnisse dieser Tests erfolgt gesondert und ist nicht Teil dieser Promotion.

Der Operationsablauf orientierte sich an dem üblichen routinemäßigen Ablauf.

Während der Operation dokumentierten wir relevante Zeitpunkte, um anschließend die Lautstärkemessungen den jeweiligen Zeitpunkten zuzuordnen. Die relevanten Zeitpunkte für die spätere Auswertung der Daten waren: Hautschnitt, Andocken des OP-Roboters, Einschnitt am Blasenhals und Abdocken des OP-Roboters.

Zudem notierten wir relevante Auffälligkeiten wie beispielsweise technische Schwierigkeiten in Zusammenhang mit SOTOS oder unplanmäßige Veränderungen im Operationsablauf.

Nach Beendigung der Hautnaht legten die Teilnehmer die EKG-Messgeräte ab. Der Abbau der Lautstärkemessgeräte sowie des SOTOS geschahen zeitgleich. Die EKG-Daten wurden per mobilen Datenträger der Klinik für Kardiologie und Pneumologie der Universitätsmedizin Göttingen zur Auslesung übergeben. 


\subsection{EKG-Messungen}

Die während der Operation mittels Vier-Kanal-Langzeit-EKG erhoben Herzfrequenzen wurden nach Beendigung des Messtages digital in das Auswertungsprogramm CardioDayC (2016, GE Healthcare, Chicago, IL, USA) übermittelt. Für jeden Messtag rechnete das Programm die Bewegungsartefakte heraus und die mittleren, maximalen und minimalen Herzfrequenzen wurden aus den Daten für die jeweilige Operation extrahiert. Eine geschulte Studienschwester der Klinik für Kardiologie und Pneumologie der Universitätsmedizin Göttingen führte diese Analyse durch. Es erfolgte die getrennte Analyse nach Funktion während der Operation (Operateur, Assistent, Anästhesisten, instrumentierende Fachkraft und Springer). Die Anonymisierung der Daten geschah durch einen Zahlencode, bestehend aus dem Datum und der Funktion. Somit war eine klare persönliche Zuordnung der Daten später nicht möglich. Zudem wurden mögliche Herzrhythmusstörungen oder Erregungsrückbildungsstörungen erfasst.

\subsection{Lautstärkemessungen}

Die Lautstärkemessungen erfolgten mittels eines Gerätes des Herstellers Voltcraft (DL161S). Hierbei handelt es sich um ein Gerät (vom Hersteller als „Datenlogger“ bezeichnet) zur Messung von Dezibel in der Wichtung A ( $\mathrm{dB}(\mathrm{A})$ ). Die Daten konnten mittels Universal Serial Bus (USB) auf einen Personal Computer (PC) übertragen werden. Dieser Datenlogger maß im Sekundentakt die Laustärke. Vor der Operation wurde der Datenlogger mittels eines Schallpegel-Kalibrators der Firma Voltcraft kalibriert. Wir setzten insgesamt zwei dieser Geräte ein (Aufbau siehe Abbildung 7).

Eines der Geräte wurde direkt am Operationsfeld mit einem Abstand von $1 \mathrm{~m}$ installiert. Das andere Gerät befand sich auch mit einem Abstand von $1 \mathrm{~m}$ vom Operateur entfernt an der Konsole. Eine kontinuierliche Messung erfolgte während der gesamten Operation. Die relevanten Zeitpunkte für die spätere Auswertung der Daten waren: Hautschnitt, Andocken, Einschnitt am Blasenhals und Abdocken. Es wurde ein Zeitfenster von +/10 Minuten um diese Zeitpunkte herum für die späteren Auswertungen herangezogen. Im Weiteren wurden die Messungen von den gemessenen Schalldruckpegeln (in dB(A)) in den Schalldruck (in $\mathrm{Pa}$ (Pascal)) umgerechnet, um für die weitere Auswertung und die Veranschaulichung der Daten eine entlogaritymierte Skala verwenden zu können.

Der Schalldruckpegel stellt eine logarithmische Messgröße für das Verhältnis des Schalldruckes zum Bezugswert dar - in diesem Fall $20 \mu \mathrm{Pa}$ in der Luft, was als die Hörschwelle des Menschen angenommen wird. 
In der folgenden Formel ist die Umrechnung dargestellt (Abbildung 12):

$$
\begin{gathered}
p(P a)=p 0 * 10^{\frac{L p(d B(A))}{20}} \\
p 0=0,00002 P a \\
d B=\text { Dezibel, } P a=\text { Pascal Lp= gemessener Schalldruckpegel } \\
p=\text { Schladdruck } \\
p 0=\text { Bezugswert (angenomme Hörschwelle des Menschen) }
\end{gathered}
$$

Abbildung 12: Umrechnung von Schalldruckpegel in Schalldruck.

\subsection{Auswertung in Bezug auf den Anästhesisten}

Aufgrund eines akuten Personalmangels konnte bei vielen Messungen der Anästhesist nicht, wie im initialen Studiensetting geplant, an dem Messablauf im vollen Umfang teilnehmen. Den Kollegen war es aufgrund des häufigen Personalwechsels nicht möglich, regelmäßig und vollständig an den Messtagen teilzunehmen. So konnte keine ausreichende Qualität an Messdaten in Bezug auf die EKG-Messungen und den Ablauf der Operation gewährleistet werden. Nach Durchführung der Messtage entschlossen wir uns somit dazu, die Messergebnisse der Anästhesisten nicht mit in die Auswertung einzubeziehen.

\section{$2.8 \quad$ Statistik}

Wie oben dargestellt, verglichen wir die unterschiedlichen Studienarme, um signifikante Unterschiede der folgenden Parameter darzustellen: Klinisch-onkologische Daten sowie Operationsparameter (siehe 2.3 Erfassung der klinischen Daten der jeweiligen Operationen), Herzfrequenzveränderung (siehe 2.5 EKG-Messungen) sowie Lautstärke im Operationssaal (siehe 2.6 Lautstärkemessung). Es erfolgte die Auswertung mittels Excel für den Macintosh (2017, Microsoft, Redmond, Washington, Vereinigte Staaten) sowie mit der Statistik Software von IBM (2017, SPSS, Inc., Chicago, IL, Version 25). Die einzelnen Daten wurden mittels Shapiro Wilks Test auf ihre Normalverteilung überprüft. Für die normalverteilten Variablen wurde ein t-Test durchgeführt. Den Wilcoxon rank sum oder der Mann-Whitney U Test verwendeten wir für die nicht normalverteilten Variablen. Kategoriale Variable werteten wir mittels Chi-Quadrat-Test aus. Das Signifikanzlevel lag bei $\mathrm{p}<$ 0,05 . 


\section{Ergebnisse}

Nachfolgend werden die Ergebnisse der Untersuchungen der SOTOS-Studie zusammengefasst. Die klinisch-onkologischen Daten und Operationsparameter, die EKG-Messungen und Lautstärkemessungen während der Operation werden getrennt nach den beiden Studienarmen dargestellt.

Zur besseren graphischen Darstellung der erhobenen Ergebnisse werden in den Unterkapiteln Box-Plots verwendet. Die Darstellung der Ergebnisse ist in all diesen Darstellungen wie folgt definiert (Mosteller und Tukey 1977): Die mittleren $50 \%$ der Daten liegen in der Box. Die Begrenzungen sind das obere und das untere Quartil, und die Länge der Box entspricht dem Interquartilsabstand (IQA). Die Länge der Antenne ist auf maximal das 1,5Fache des Interquartilsabstands (1,5×IQA) festgelegt. Dabei endet die Antenne nicht genau nach dieser Länge, sondern bei dem Wert aus den Daten, der noch innerhalb des 1,5Fachen IQA liegt. Die Länge der Antennen wird also durch die Datenwerte und nicht allein durch den Interquartilsabstand bestimmt. Somit sind die Antennen nicht auf beiden Seiten gleich lang. Gibt es keine Werte außerhalb der Grenze von 1,5×IQA, wird die Länge der Antenne durch den maximalen und minimalen Wert definiert. Die zwischen 1,5×IQA und $3 \times$ IQA liegenden Ausreißer werden als „milde“ Ausreißer bezeichnet und Werte, die über $3 \times$ IQA liegen, als „extreme“ Ausreißer.

\subsection{Klinisch-onkologische Daten und Operationsparameter}

Die einzelnen Studienarme werden im Folgenden im Hinblick auf klinisch-onkologische Parameter und prä-, intra- sowie postoperative Unterschiede verglichen.

Die beiden Studienpopulationen unterschieden sich statistisch nicht bezüglich des Patientenalters (Mittelwert: 68,2 Jahre Standardabweichung: \pm 6,4 Jahre, $p=0,201$, Abbildung 13). 


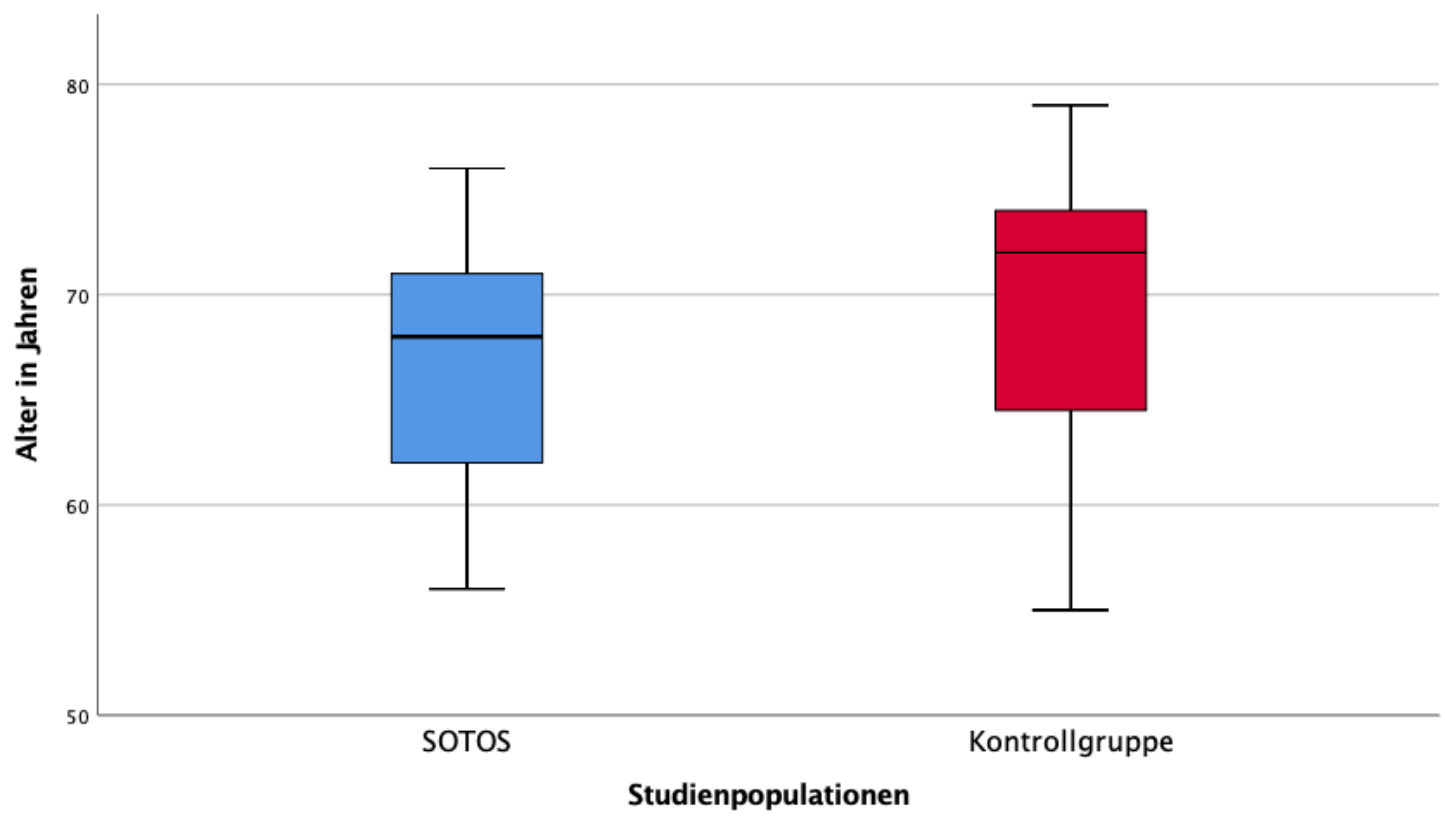

Abbildung 13: Vergleich des Alters der Studienpopulationen.

Im Folgenden werden die präoperativen klinischen Daten (Prostatagröße, Anzahl voroperierter Patienten) der Patienten verglichen (Tabelle 3).

Tabelle 3: Vergleich der präoperativen klinischen Parameter der Studienarme (*unabhängiger t-Test; ${ }^{* *}$ Chi-Quadrat-Test; ***jegliche abdominelle Operation in der Vorgeschichte).

\begin{tabular}{l|l|l|l} 
& SOTOS $(n=16)$ & Kontrollgruppe $(n=16)$ & -Wert \\
\hline $\begin{array}{l}\text { Prostatagröße im Mittel in ml } \\
( \pm \text { Standardabweichung) }\end{array}$ & $42,63( \pm 23,7)$ & $47,13( \pm 31,1)$ & $\mathrm{p}=0,648^{*}$ \\
$\begin{array}{l}\text { Anzabl voroperierter Patienten*** } \\
\text { ( }\end{array}$ & $12(75 \%)$ & $5(31,3 \%)$ & $\mathrm{p}=0,09^{* *}$
\end{tabular}

Die Prostatagröße war in beiden Studienarmen $(42,63$ ( \pm 23,7) in der SOTOS-Gruppe und 47,13 ( \pm 31,1) in der Kontrollgruppe) vergleichbar und unterschied sich nicht statistisch relevant. In der SOTOS-Gruppe waren im Vergleich zur Kontrollgruppe mehr Patienten voroperiert, ohne dass der Unterschied das vordefinierte Signifikanzniveau erreichte. Diese Voroperationen spiegeln sich jedoch nicht in einer unterschiedlichen Operationszeit (4 h $6 \mathrm{~min} \pm 59 \mathrm{~min}$ in der SOTOS-Gruppe und $4 \mathrm{~h} 20 \mathrm{~min} \pm 35 \mathrm{~min}$ in der Kontrollgruppe, Tabelle 5) wider.

Die präoperativ erhobenen onkologischen Daten sind in Tabelle 4 dargestellt. 
Tabelle 4: Vergleich der präoperativen onkologischen Parameter der Studienarme $\left({ }^{*} \mathrm{Chi}-\right.$ Quadrat-Test; ** Mann-Whitney U Test).

\begin{tabular}{l|l|l|l} 
& SOTOS $(n=16)$ & Kontrollgruppe $(n=16)$ & $p$-Wert \\
\hline $\begin{array}{l}\text { Gleason-Score n (\%) } \\
6\end{array}$ & $5(31,3)$ & $6(37,5)$ & \\
$7 a$ & $5(31,3)$ & $3(18,8)$ & $\mathrm{p}=0,763^{*}$ \\
$7 b$ & $5(31,3)$ & $5(31,3)$ & $\mathrm{p}=0,48^{*}$ \\
8 & $1(6,25)$ & $2(12,5)$ & $\mathrm{p}=1^{*}$ \\
iPSA im Mittel in ng/ml & $12,55( \pm 13,02)$ & $8,81( \pm 3,89)$ & $\mathrm{p}=0,564^{*}$ \\
+ (- Standardabweichung) & & $\mathrm{p}=0,53)^{* *}$ \\
D'Amico n (\%) & $5(31,3)$ & $5(31,3)$ & \\
niedrig & $9(56,3)$ & $9(56,3)$ & $\mathrm{p}=1^{*}$ \\
mittel & $2(12,5)$ & $2(12,5)$ & $\mathrm{p}=1^{*}$ \\
hoch & & $\mathrm{p}=1^{*}$
\end{tabular}

Hinsichtlich der präoperativen onkologischen Daten zeigten sich zwei vergleichbare Gruppen. Statistisch konnte kein signifikanter Unterschied zwischen den iPSA-Werten (PSASerumwert bei Diagnose des Prostatakarzinoms) der Patienten festgestellt werden. In den oben dargestellten Daten zeigt sich in den präoperativ durch die Stanzbiopsien erhobenen Gleason-Score kein statistisch nachweisbarer Unterschied der zwei Studiengruppen. Die meisten Patienten befanden sich entsprechend der Klassifikation nach D'Amico in der mittleren Risikogruppe (jeweils neun Patienten in der SOTOS- bzw. Kontrollgruppe; $\mathrm{p}=1$ ). Im Weiteren wurden die intraoperativen erhobenen Parameter analysiert (Tabelle 5).

Intraoperative Besonderheiten waren als erhöhter Blutverlust (über $300 \mathrm{ml}$ ) oder Verletzungen des Dünn- bzw. Dickdarms insbesondere des Rektums, der Gefäße oder der Nerven definiert. Schwierige Operationsverhältnisse waren durch abdominelle Voroperationen oder Verwachsungen im Situs dokumentiert worden. 
Tabelle 5: Vergleich der intraoperativen Parameter der Studienarme (*unabhängiger T-Test; ** Chi-Quadrat-Test).

\begin{tabular}{|c|c|c|c|}
\hline & $\begin{array}{l}\text { SOTOS } \\
(n=16)\end{array}$ & $\begin{array}{l}\text { Kontrollgruppe } \\
(n=16)\end{array}$ & $p$-Wert \\
\hline $\begin{array}{l}\text { Mittlere OP-Dauer in Stunden (b) und } \\
\text { Minuten (m) (+/-Standardabweichung) }\end{array}$ & $\begin{array}{l}4 \mathrm{~h} 6 \mathrm{~min} \\
\pm 59 \mathrm{~min}\end{array}$ & $\begin{array}{l}4 \text { h } 20 \mathrm{~min} \\
\pm 35 \mathrm{~min}\end{array}$ & $\mathrm{p}=0,425^{*}$ \\
\hline $\begin{array}{l}\text { Operationen mit Nerverbalt und Schnell- } \\
\text { schnitt } n(\%)\end{array}$ & $11(68,8)$ & $10(62,5)$ & $\mathrm{p}=0,48^{* *}$ \\
\hline Besonderbeiten/schwierige OP $n(\%)$ & $5(31,3)$ & $6(37,5)$ & $\mathrm{p}=0,763^{* *}$ \\
\hline
\end{tabular}

Ein Vergleich der operativen Parameter ergab keinen statistisch signifikanten Unterschied. Somit verliefen die Operationen hinsichtlich dieser Variablen in vergleichbarer Weise und die Anwendung des SOTOS hatte keinen Einfluss auf diese Parameter.

Die postoperativ erhobenen klinischen Parameter sind in Tabelle 6 dargestellt.

Tabelle 6: Vergleich der postoperativen klinischen Parameter der Studienarme (*unabhängiger t-Test; **Chi-Quadrat-Test).

\begin{tabular}{l|l|l|l} 
& $\begin{array}{l}\text { SOTOS-Gruppe } \\
(n=16)\end{array}$ & $\begin{array}{l}\text { Kontrollgruppe } \\
(n=16)\end{array}$ & p-Wert \\
\hline $\begin{array}{l}\text { Postoperative Komplikationen n (\%) } \\
\text { Postoperative Infektionen (\%) }\end{array}$ & $4(25)$ & $1(6,3)$ & $\mathrm{p}=0,18^{* *}$ \\
Lymphozelen n (\%) & $3(18,3)$ & $1(6,3)$ & $\mathrm{p}=0,317^{* *}$ \\
Mittlere Liegedauer in Tagen & $9(25)$ & $1(6,3)$ & $\mathrm{p}=0,18^{* *}$ \\
(+/-Standardabweichung) & $9,9 \pm 3,6$ & $\mathrm{p}=0,425^{*}$
\end{tabular}

Die postoperativen Infektionen, Lymphozelen und Komplikationen wie z. B. Nachblutungen, waren in der SOTOS-Gruppe tendenziell erhöht. Auch zeigte sich in der SOTOSGruppe eine längere Liegedauer, ohne dass diese Unterschiede der Parameter das vordefinierte Signifikanzniveau erreichten.

Die postoperativ erhobenen onkologischen Daten werden in Tabelle 7 präsentiert. 
Tabelle 7: Vergleich der postoperativ erhobenen onkologischen Parameter der Studienarme (*Chi-Quadrat-Test; **es gibt nicht genügend Fälle für eine statistische Auswertung).

\begin{tabular}{l|l|l|l} 
& SOTOS $(n=16)$ & Kontrollgruppe (n=16) & -Wert \\
\hline Gleason-Score n (\%) & & & \\
6 & $3(18,8)$ & $2(12,5)$ & $\mathrm{p}=0,655^{*}$ \\
$7 a$ & $9(56,3)$ & $5(31,3)$ & $\mathrm{p}=0,285^{*}$ \\
$7 b$ & $2(12,5)$ & $7(43,8)$ & $\mathrm{p}=0,96^{*}$ \\
8 & $2(12,5)$ & $1(6,3)$ & $\mathrm{p}=0,564^{*}$ \\
9 & 0 & $1(6,3)$ & $* *$ \\
R1-Resektion $n(\%)$ & $1(6,3)$ & $3(18,8)$ & $\mathrm{p}=0,317^{*}$ \\
N+-Befund $n(\%)$ & $0(-)$ & $0(-)$ & $* *$ \\
Upgrading (5) & $6(37,5)$ & $8(50 \%)$ & $\mathrm{p}=0,593^{*}$
\end{tabular}

Insgesamt zeigte sich eine Erhöhung des Gleason-Score („Upgrading“) in den beiden Studienarmen (acht Patienten in der Kontrollgruppe und sechs Patienten in der SOTOSGruppe), welche nicht signifikant unterschiedlich zwischen den beiden Armen war. Unterschiede bezüglich des Resektionsstatus zwischen beiden Interventionsgruppen mit einer höheren Rate an R1-Resektionen in der Kontroll-Gruppe erreichten das vordefinierte Signifikanzniveau nicht.

\subsection{EKG-Messungen}

Es wurde eine Gesamtgruppenanalyse zur Detektion möglicher Unterschiede zwischen den Studienpopulationen durchgeführt. Im Folgenden werden die Herzfrequenzen für die Gesamtkohorte dargestellt (Abbildung 14, siehe nächste Seite).

Es zeigte sich kein statistisch signifikanter Unterschied zwischen den einzelnen OPFunktionen (i.e. Operateur, OP-Assistent, Instrumentierende Fachkraft, Springer) hinsichtlich der mittleren Herzfrequenz. Als normale Herzfrequenz eines Erwachsenen wird eine Frequenz zwischen 50-100 Schlägen pro Minute angesehen (Lentner 1990, S. 187-197). Die mittleren Herzfrequenzen der Operateure (Mittelwert: 81,3 \pm 7,1 Schläge/min), des OPAssistenten (Mittelwert: 87,7 \pm 10,9 Schläge/min), der instrumentierenden Fachkraft (Mittelwert: 86,9 \pm 7,4 Schläge/min) und des Springers (Mittelwert: 90,8 \pm 7,4 Schläge/min) lagen im normalen Bereich und unterschieden sich nicht statistisch signifikant $(p=0,33)$. 
In der Abbildung 15 (siehe nächste Seite) werden die mittleren Herzfrequenzen der einzelnen OP-Funktionen getrennt nach Studienarmen demonstriert.

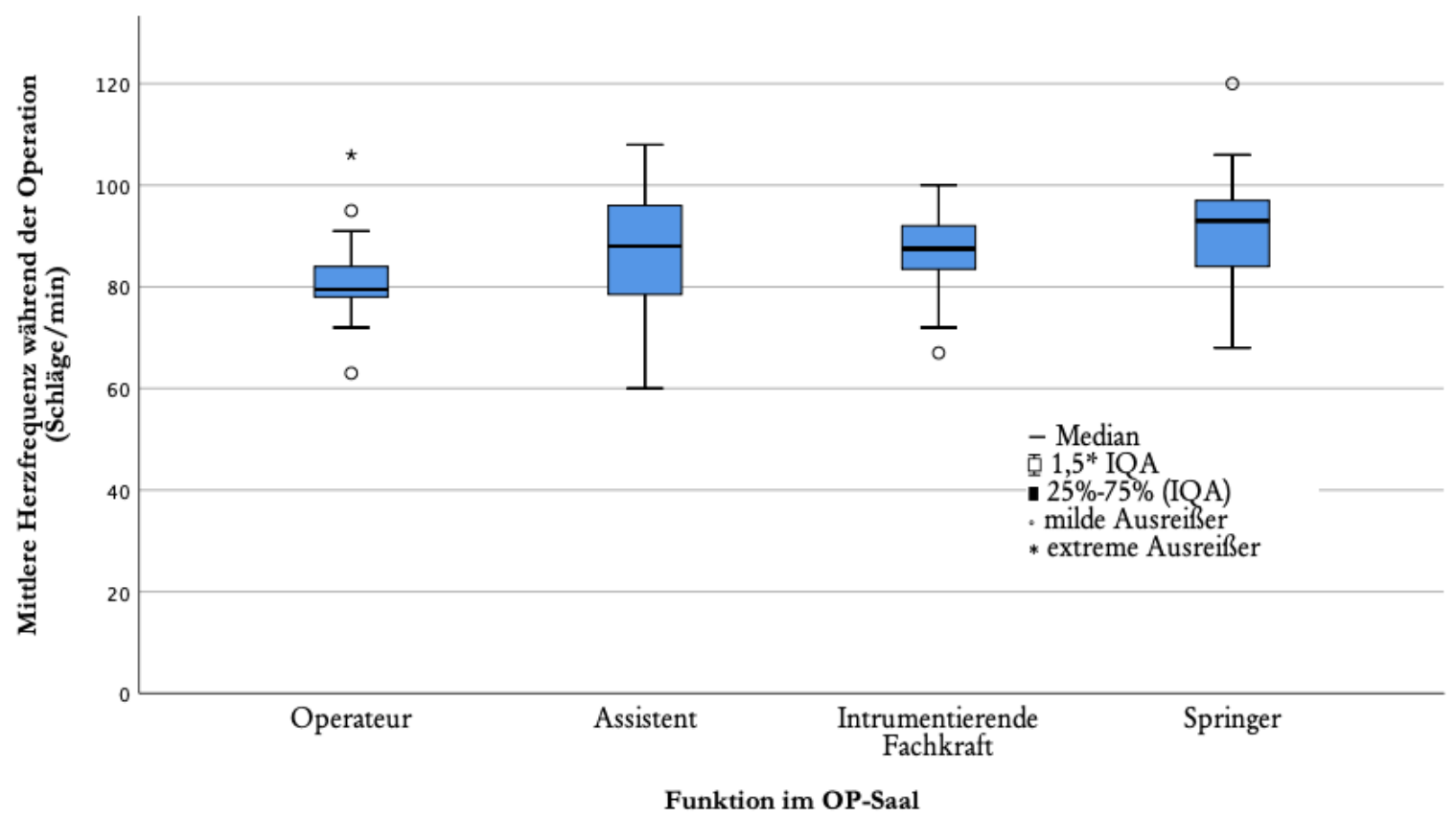

Abbildung 14: Vergleich der mittleren Herzfrequenzen der Gesamtkohorte. (IQA= Interquartilsabstand).

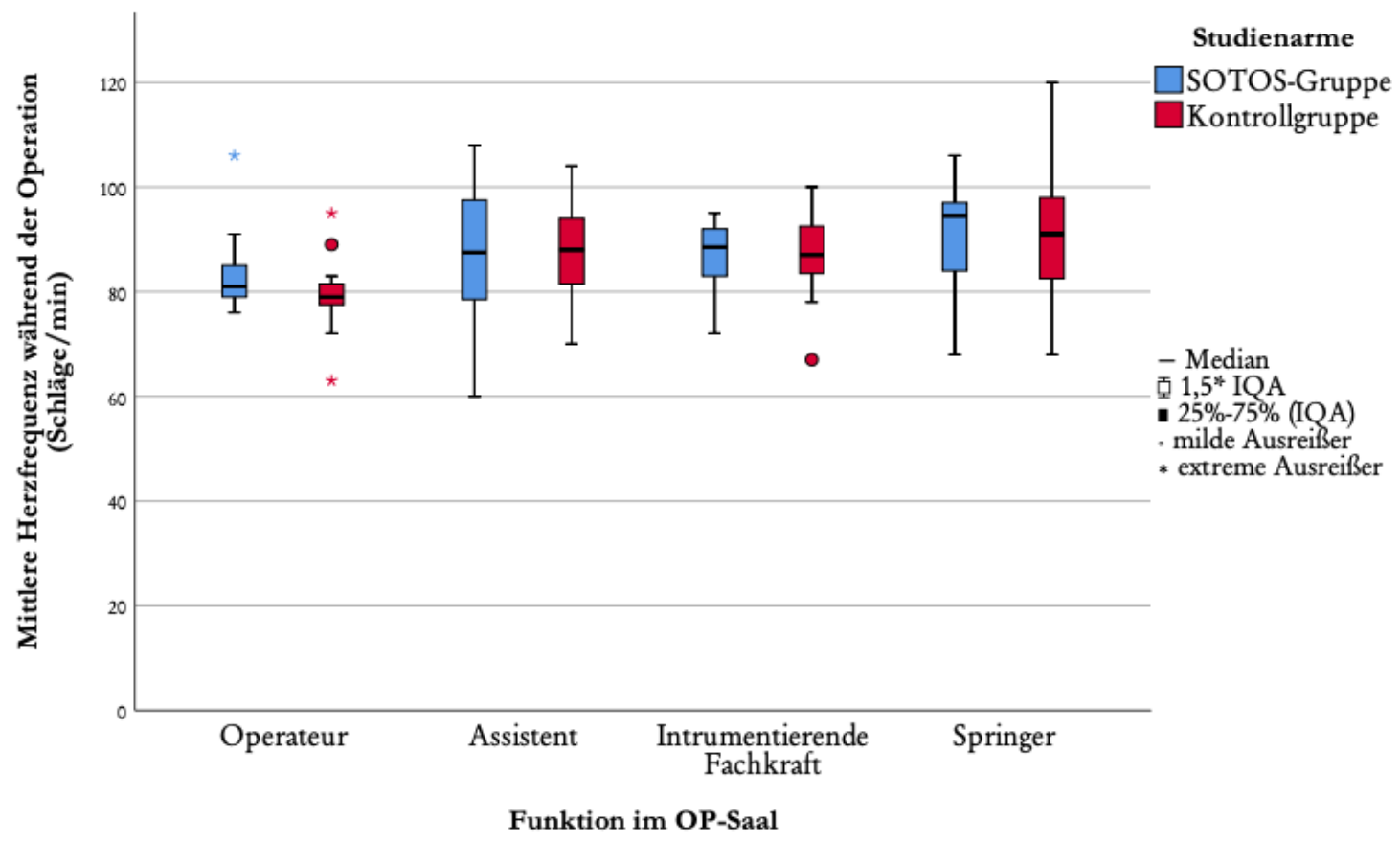

Abbildung 15: Vergleich der mittleren Herzfrequenzen der einzelnen OP-Funktionen (i.e. Operateur, Assistent, Instrumentierende Fachkraft, Springer) getrennt nach Studiengruppe (IQA= Interquartilsabstand). 
Im Vergleich zeigte sich kein signifikanter Unterschied der Gruppen bezüglich der mittleren Herzfrequenzen bei den einzelnen Funktionen im OP-Saal. Auch in dem direkten Vergleich der SOTOS- und Kontrollgruppe bei den mittleren Herzfrequenzen liegen die Werte innerhalb des oben genannten Referenzbereichs für einen normalen Erwachsenen. Die Messwerte in den mittleren Herzfrequenzen der Operateure (Mittelwert für SOTOS: 83,2 \pm 7,2 Schläge/min, Kontrolle: 79,50 \pm 6,8 Schläge/min; $\mathrm{p}=0,15$ ), des OP-Assistenten (Mittelwert für SOTOS: 87,44 \pm 12,1 Schläge/min, Kontrolle: 87,9 \pm 9,9 Schläge/min $\mathrm{p}=0,899$ ), der instrumentierenden Fachkraft (Mittelwert für SOTOS: 86,88 \pm 6,9 Schläge/min, Kontrolle: 86,9 \pm 8,1 Schläge/min; $\mathrm{p}=0,981$ ) und des Springers (Mittelwert für SOTOS: 90,9 \pm 9,3 Schläge/min, Kontrolle: 90,8 \pm 13,2 Schläge/min; $p=0,963)$ unterschieden sich nicht statistisch signifikant.

Zusätzlich untersuchten wir die minimalen (Abbildung 16) und maximalen (Abbildung 17) Herzfrequenzen der einzelnen OP-Funktionen getrennt nach Studienarmen.

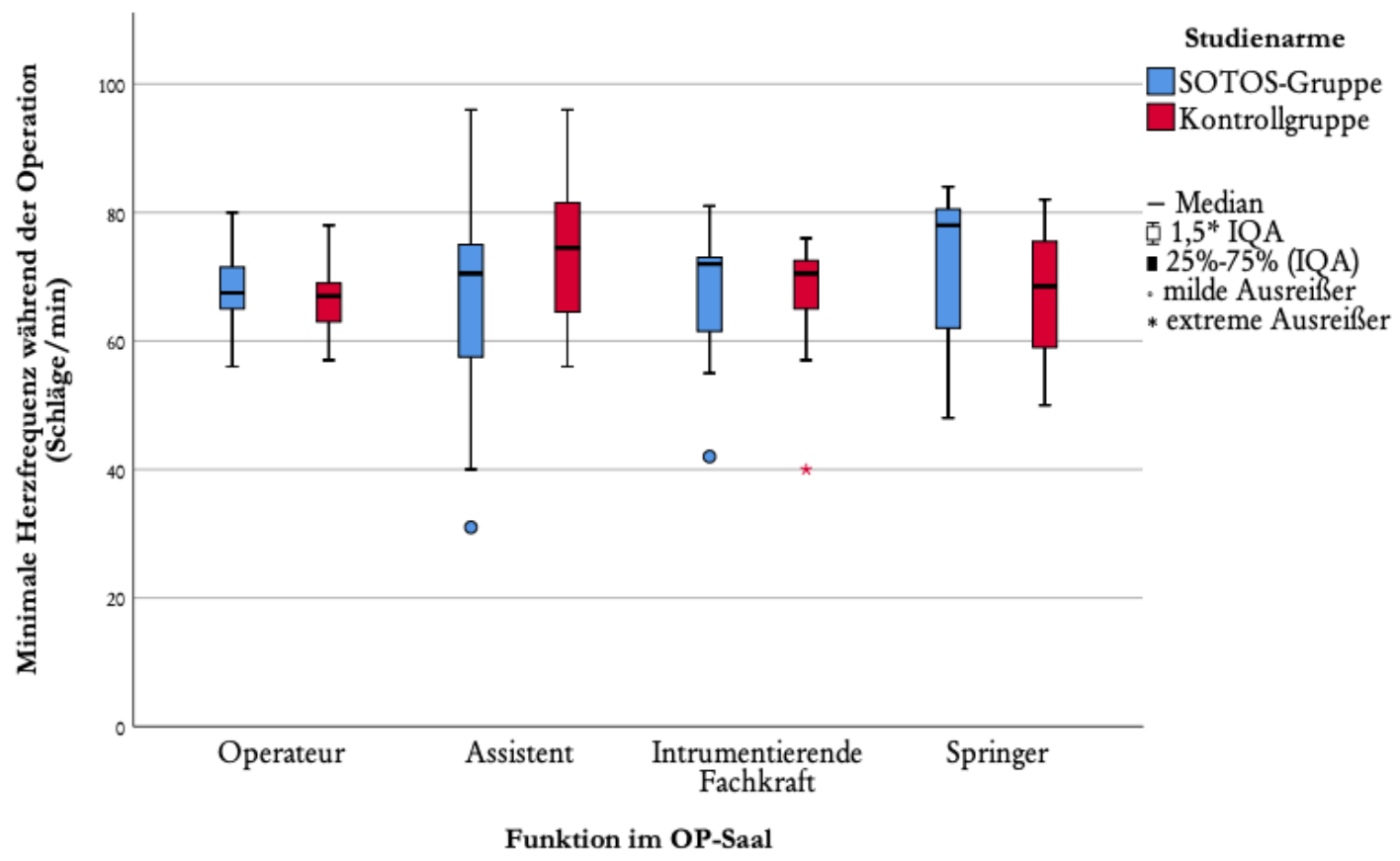

Abbildung 16: Vergleich der minimalen Herzfrequenzen der einzelnen OP-Funktionen (i.e. Operateur, Assistent, Instrumentierende Fachkraft, Springer) getrennt nach Studiengruppe (IQA= Interquartilsabstand).

Im Vergleich der SOTOS- und Kontrollgruppe bei den minimalen Herzfrequenzen liegen die Werte innerhalb des oben genannten Referenzbereichs für einen normalen Erwachsenen. Die Messwerte in den minimalen Herzfrequenzen der Operateure (Mittelwert für SOTOS: $67,75 \pm$ 6,1 Schläge/min, Kontrolle: $66,1 \pm$ 5,2 Schläge/min; $\mathrm{p}=0,421)$, des OPAssistenten (Mittelwert für SOTOS: 67,13 \pm 16,8 Schläge/min, Kontrolle: 74,25 \pm 12,7 Schläge/min; p=0,185), der instrumentierenden Fachkraft (Mittelwert für SOTOS: 67,8 \pm 
10,4 Schläge/min, Kontrolle: 67,4 \pm 9,2 Schläge/min; $\mathrm{p}=0,915)$ und des Springers (Mittelwert für SOTOS: 71,8 \pm 11,2 Schläge/min, Kontrolle: $67,7 \pm$ 9,9 Schläge/min; $\mathrm{p}=0,279$ ) unterschieden sich nicht statistisch signifikant.

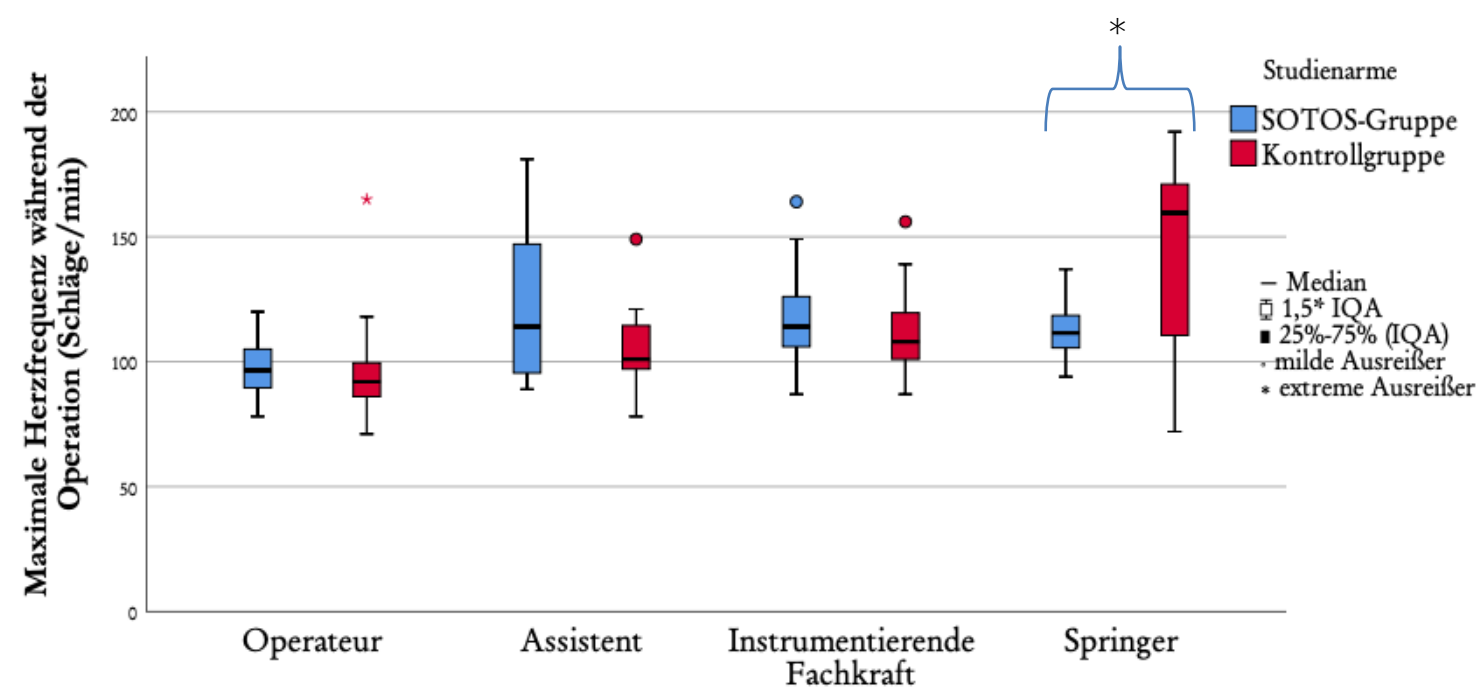

Abbildung 17: Vergleich der maximalen Herzfrequenzen der einzelnen OP-Funktionen (i.e. Operateur, Assistent, Instrumentierende Fachkraft, Springer) getrennt nach Studiengruppe (IQA= Interquartilsabstand, *statistisch signifikant).

In diesen Untersuchungen zeigten sich bei den Operateuren, Assistenten und der instrumentierenden Fachkraft keine statistisch signifikanten Unterschiede bezüglich der maximalen Herzfrequenzen zwischen den Gruppen. Die Betrachtung der maximalen Herzfrequenzen zeigt jedoch, dass die mittleren Maximalwerte außerhalb des normalen Herzfrequenzspektrums für Erwachsene liegen und somit es in manchen Situationen durchaus zu erhöhten Frequenzen während der Operation kommt. Einzig die Gruppe der Operateure zeigte diesen Fakt nicht. Die Messwerte in den maximalen Herzfrequenzen der Operateure (Mittelwert für SOTOS: 97,7,2 \pm 12,9 Schläge/min, Kontrolle: 96,63 \pm 21,3 Schläge/min; $\mathrm{p}=0,866$ ), der OP-Assistenten (Mittelwert für SOTOS: 120,7 \pm 28,2 Schläge/min, Kontrolle: 105,2 \pm 16 Schläge/min; $\mathrm{p}=0,066)$ und der instrumentierenden Fachkraft (Mittelwert für SOTOS: 118,8 \pm 5,1 Schläge/min, Kontrolle: 113,31 \pm 4,5 Schläge/min; $p=0,981)$ unterschieden sich nicht statistisch signifikant. In der Subgruppe der Springer (Mittelwert für SOTOS: 111,6 \pm 12 Schläge/min, Kontrolle: 143,8 \pm 35 Schläge/min; $p<0,001)$ zeigte sich eine signifikante Senkung der maximalen Herzfrequenz durch SOTOS.

Im Weiteren untersuchten wir die Subgruppe der einzelnen Operateure auf intraindividuelle Unterschiede mit oder ohne SOTOS-Anwendung. Es zeigten sich keine signifikanten Unterschiede hinsichtlich der mittleren, minimalen und maximalen Herzfrequenzen (Abbildung 18, 19 und 20, siehe Seite 30 und 31). 


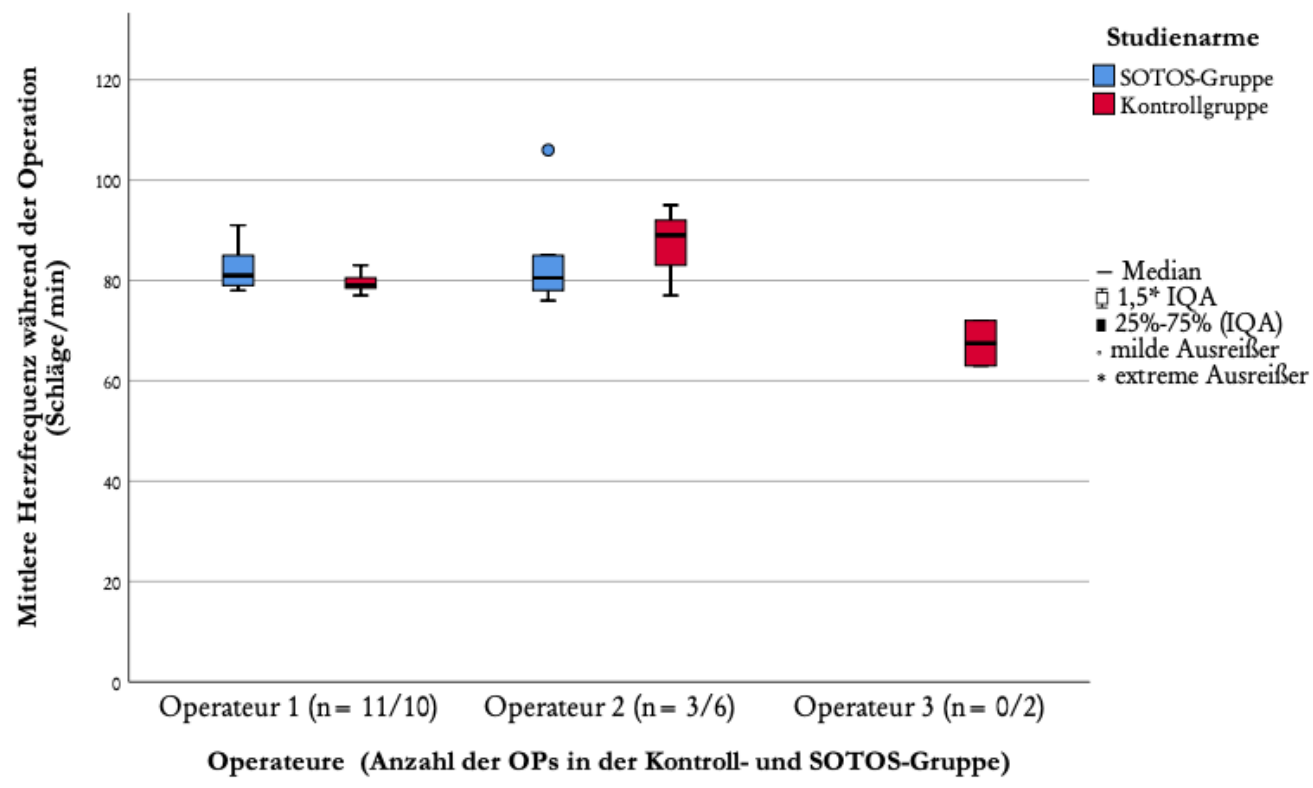

Abbildung 18: Vergleich der mittleren Herzfrequenzen der einzelnen Operateure getrennt nach Studiengruppe (IQA= Interquartilsabstand).

Die mittleren Herzfrequenzen des Operateur 1 (Mittelwert für SOTOS: 82,5 \pm 4,3 Schläge/min, Kontrolle: 79,6 \pm 1,8 Schläge/min; $\mathrm{p}=0,058$ ), Operateur 2 (Mittelwert für SOTOS: 84,3 \pm 11,1 Schläge/min, Kontrolle: $87 \pm$ 9,2 Schläge/min; $\mathrm{p}=0,73)$ und Operateur 3 (Mittelwert für die Kontrolle: 67,5 \pm 4,5 Schläge/min) unterschieden sich nicht statistisch signifikant.

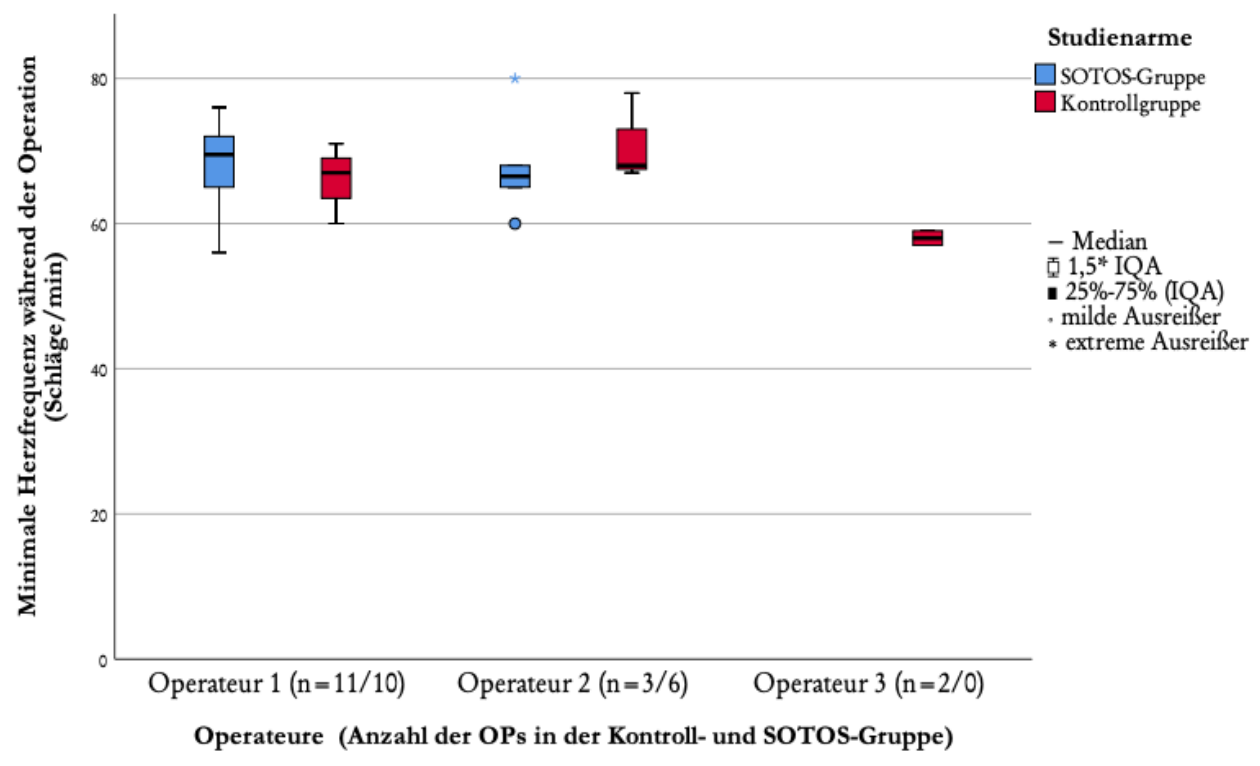

Abbildung 19: Vergleich der minimalen Herzfrequenzen der einzelnen Operateure getrennt nach Studiengruppe (IQA = Interquartilsabstand). 
Die minimalen Herzfrequenzen des Operateurs 1 (Mittelwert für SOTOS: 67,8 \pm 6 Schläge/min, Kontrolle: 66,27 $\pm 3,5$ Schläge/min p=0,481), Operateur 2 (Mittelwert für SOTOS: 67,67 \pm 6,7 Schläge/min, Kontrolle: $71 \pm 6,1$ Schläge/min $\mathrm{p}=0,492)$ und Operateur 3 (Mittelwert für die Kontrolle: $58 \pm$ 1,4 Schläge/min) unterschieden sich nicht statistisch signifikant.

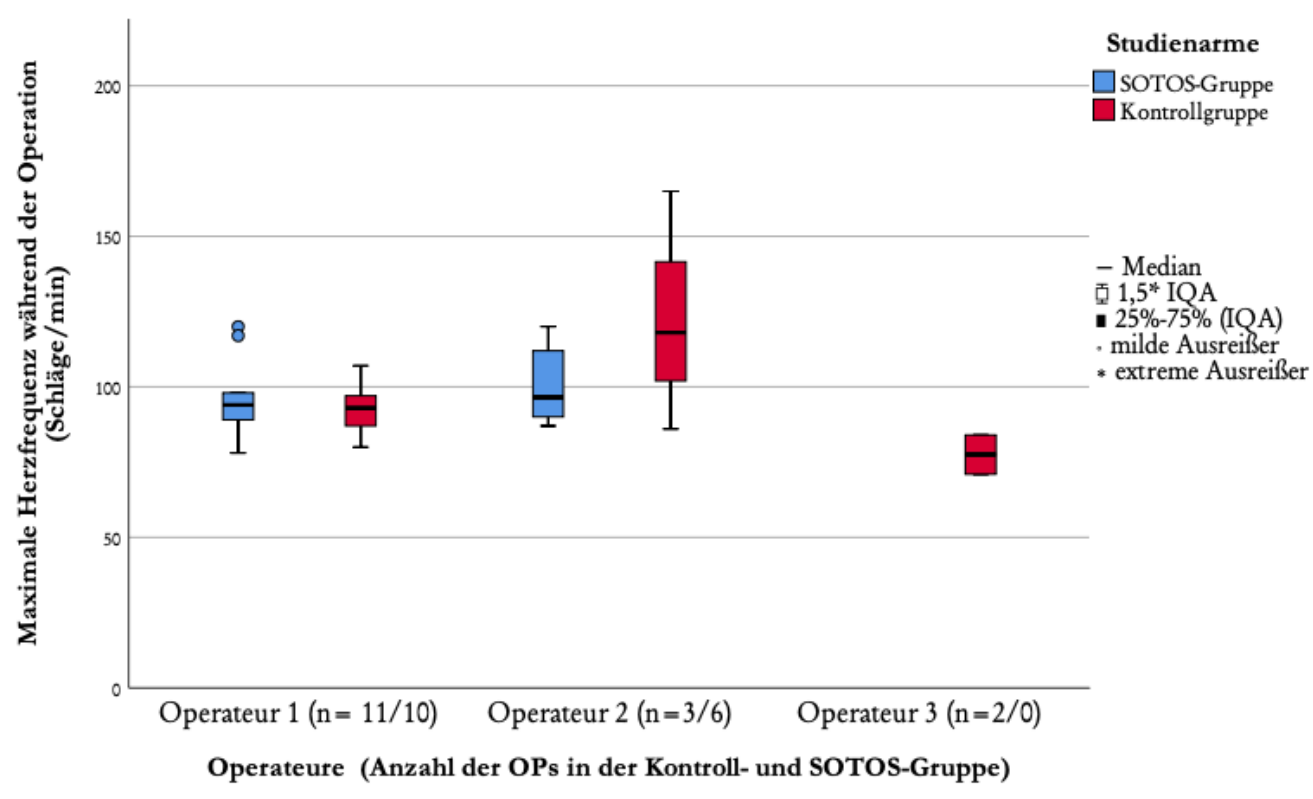

Abbildung 20: Vergleich der maximalen Herzfrequenzen der einzelnen Operateure getrennt nach Studiengruppe (IQA = Interquartilsabstand).

Die minimalen Herzfrequenzen des Operateurs 1 (Mittelwert für SOTOS: 96,1 \pm 13,3 Schläge/min, Kontrolle: 92,9 \pm 7,8 Schläge/min p=0,506), Operateur 2 (Mittelwert für SOTOS: 100,3 \pm 12,9 Schläge/min, Kontrolle: $123 \pm$ 39,7 Schläge/min p=0,222) und Operateur 3 (Mittelwert für die Kontrolle: 77,5 \pm 9,2 Schläge/min) unterschieden sich nicht statistisch signifikant.

In den drei o. g. Abbildungen zeigten sich zwischen den Herzfrequenzen der einzelnen Operateure keine signifikanten Unterschiede. Zwei Operateure führten jeweils Operationen in beiden Studiengruppen durch. Operateur 3 führte zwei Operationen in der Kontrollgruppe durch. In einer Subanalyse zeigte sich in den Abbildungen 18, 19 und 20 eine tendenzielle Senkung der minimalen, mittleren und maximalen Herzfrequenzen bei Operateur 2. Dieser Effekt konnte aufgrund der geringen Operationszahlen des Operateurs nicht statistisch ausgewertet werden.

Zudem fiel eine hohe Anzahl von Artefakten während der Auswertungen der Rohdaten auf. Somit war eine minutengenaue Bewertung der Herzfrequenzen nicht möglich. 


\subsection{Lautstärkemessungen}

Nachfolgend sind die Vergleiche der Lautstärkemessungen für SOTOS- und Kontrollgruppe dargestellt.

\subsubsection{Lautstärkemessungen über die gesamte Operation an beiden Messorten}

Der nachfolgende Box-Plot bildet den Vergleich der Lautstärke beider Messorte (OPGebiet und Konsole) zwischen den Studiengruppen über den gesamten Operationszeitraum ab (Abbildung 21). Zur besseren Auswertbarkeit wurden jeweils die Mediane der vier verschiedenen Messzeitpunkte (Hautschnitt, das Andocken des OP-Roboters, der Einschnitt am Blasenhals und das Abdocken des Roboters) der 32 Messtage für die Berechnungen verwendet.

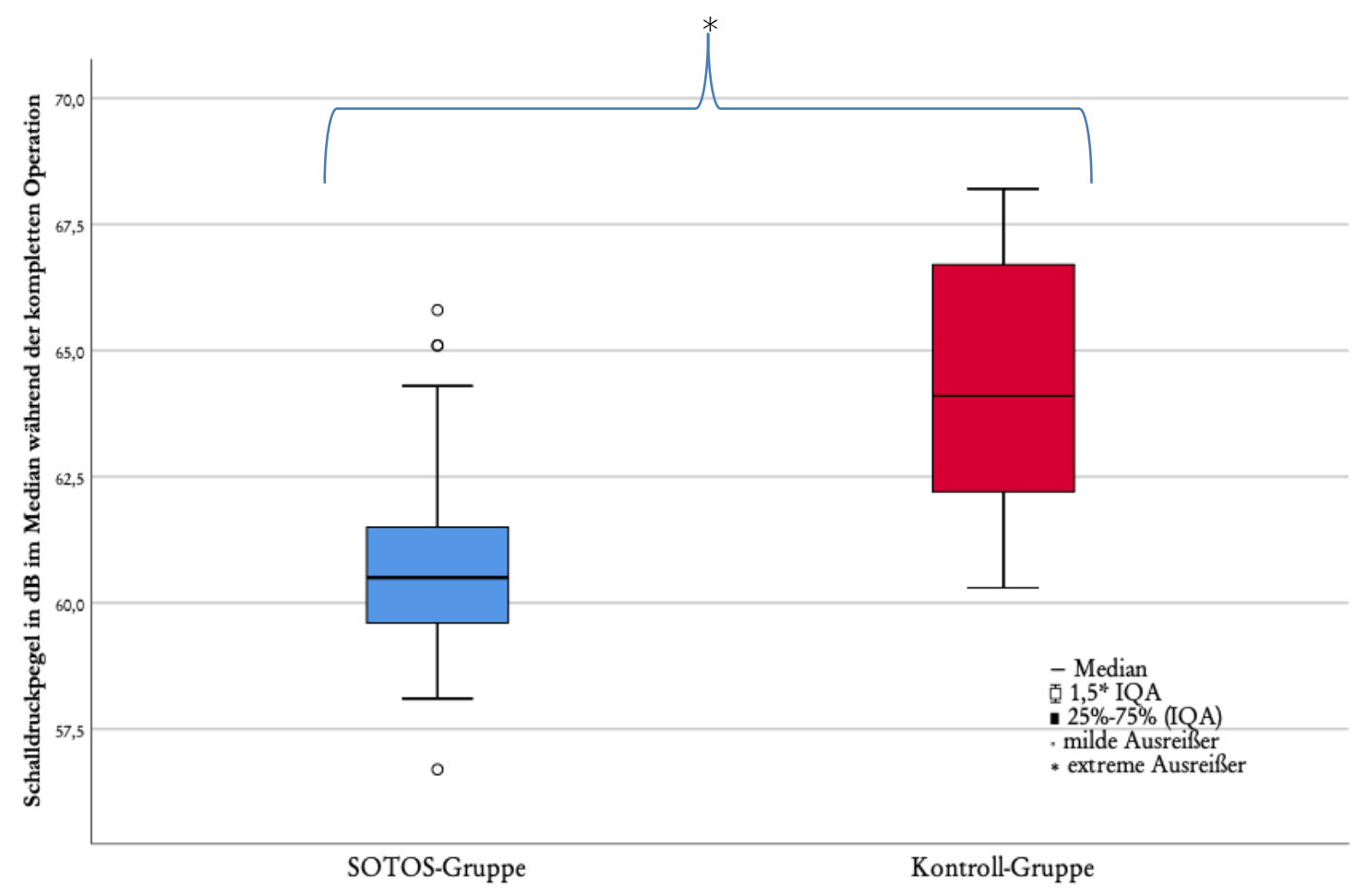

Abbildung 21: Vergleich der Schalldruckpegel (in dB) zwischen den beiden Studiengruppen (IQA= Interquartilsabstand, * statistisch signifikant).

Im Mittel zeigte sich insgesamt eine signifikante Senkung des medianen Schalldruckpegels um 3,6 dB durch SOTOS (Standardabweichung \pm 1,6 dB) im Vergleich zur Kontrollgruppe (Standardabweichung $\pm 2,3 \mathrm{~dB} ; \mathrm{p}<0,001)$.

Zum Vergleich entspricht eine Erhöhung des Schalldruckpegels um 3 dB der Verdopplung der Schallintensität, eine Erhöhung des Schalldruckpegels um $6 \mathrm{~dB}$ der Verdopplung des Schalldrucks. Eine Erhöhung des Schalldruckpegels um 10 dB soll der Empfindung "doppelte Lautstärke" entsprechen. 
Weiterführend wurden die Messungen in den Schalldruck umgerechnet (Abbildung 22). Auch hierfür erfolgte zur besseren Auswertbarkeit die Verwendung der Mediane der vier Messzeitpunkte.

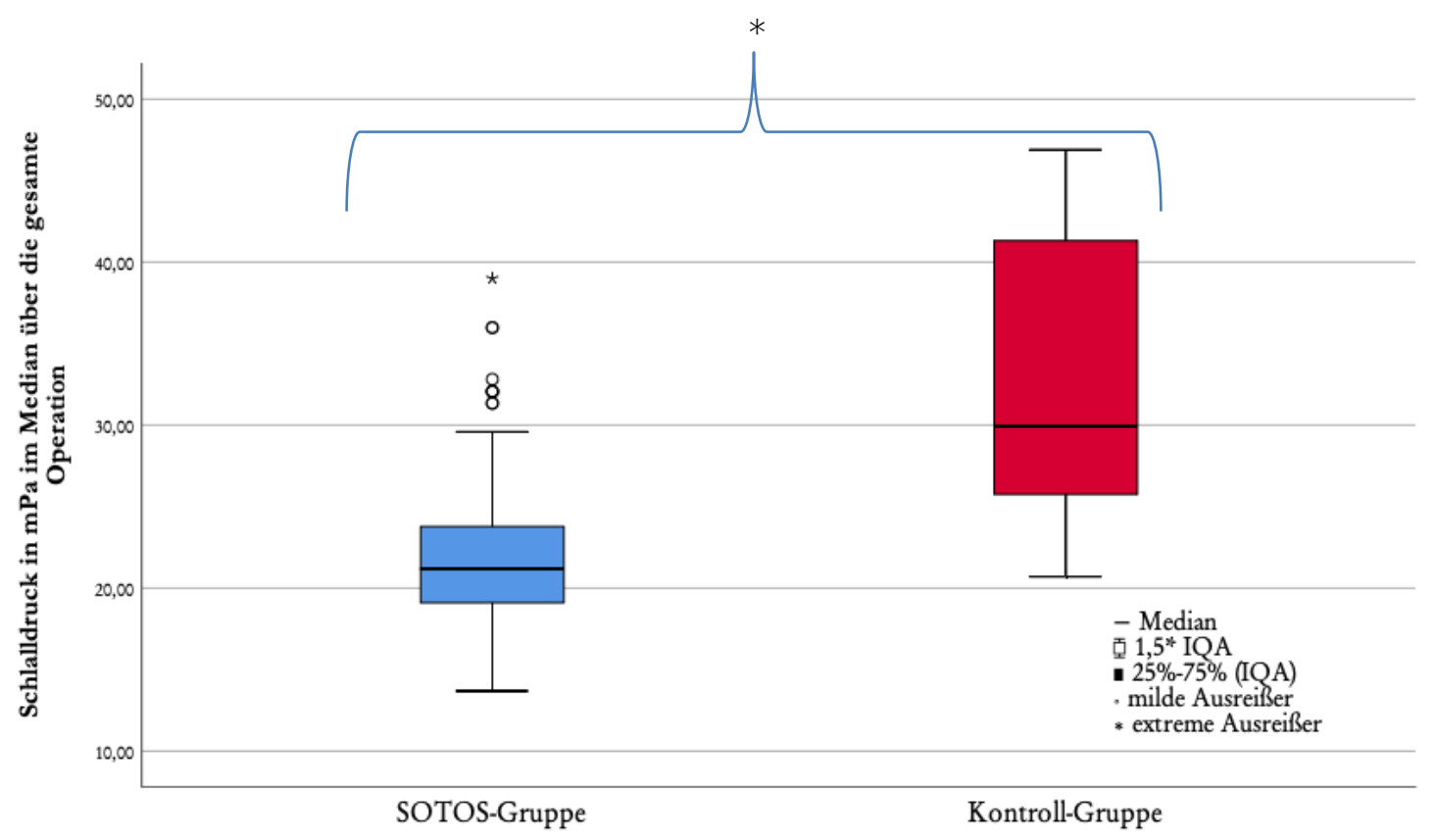

Abbildung 22: Vergleich des Schalldruckes (in $\mathrm{mPa}$ ) zwischen den beiden Studiengruppen (IQA= Interquartilsabstand, * statistisch signifikant).

Hier zeigte sich eine signifikante Senkung des medianen Schalldruckes im Mittel um 8,7 $\mathrm{mPa}$ durch den Einsatz von SOTOS (Standardabweichung \pm 4,3 $\mathrm{mPa}$ ) im Vergleich zur Kontrollgruppe (Standardabweichung $\pm 8,3 \mathrm{mPa} ; \mathrm{p}<0,001$ ). Vergleichsweise erzeugt ein Fernseher (in $1 \mathrm{~m}$ Entfernung) einen Schalldruck von $2 \mathrm{mPa}$ und ein PKW (in $1 \mathrm{~m}$ Entfernung) einen Schalldruck von $20 \mathrm{mPa}$.

\subsubsection{Messergebnisse des Schalldruckpegels an vier verschiedenen Messzeitpunkten}

In einer weiterführenden Analyse untersuchten wir an vier verschiedenen Zeitpunkten während der Operation Unterschiede zwischen den Studienarmen.

In der Abbildung 23 wird der Schalldruckpegel (in dB) am OP-Feld (mit $1 \mathrm{~m}$ Abstand) zu den jeweiligen Messzeitpunkten in beiden Gruppen gegenübergestellt. 


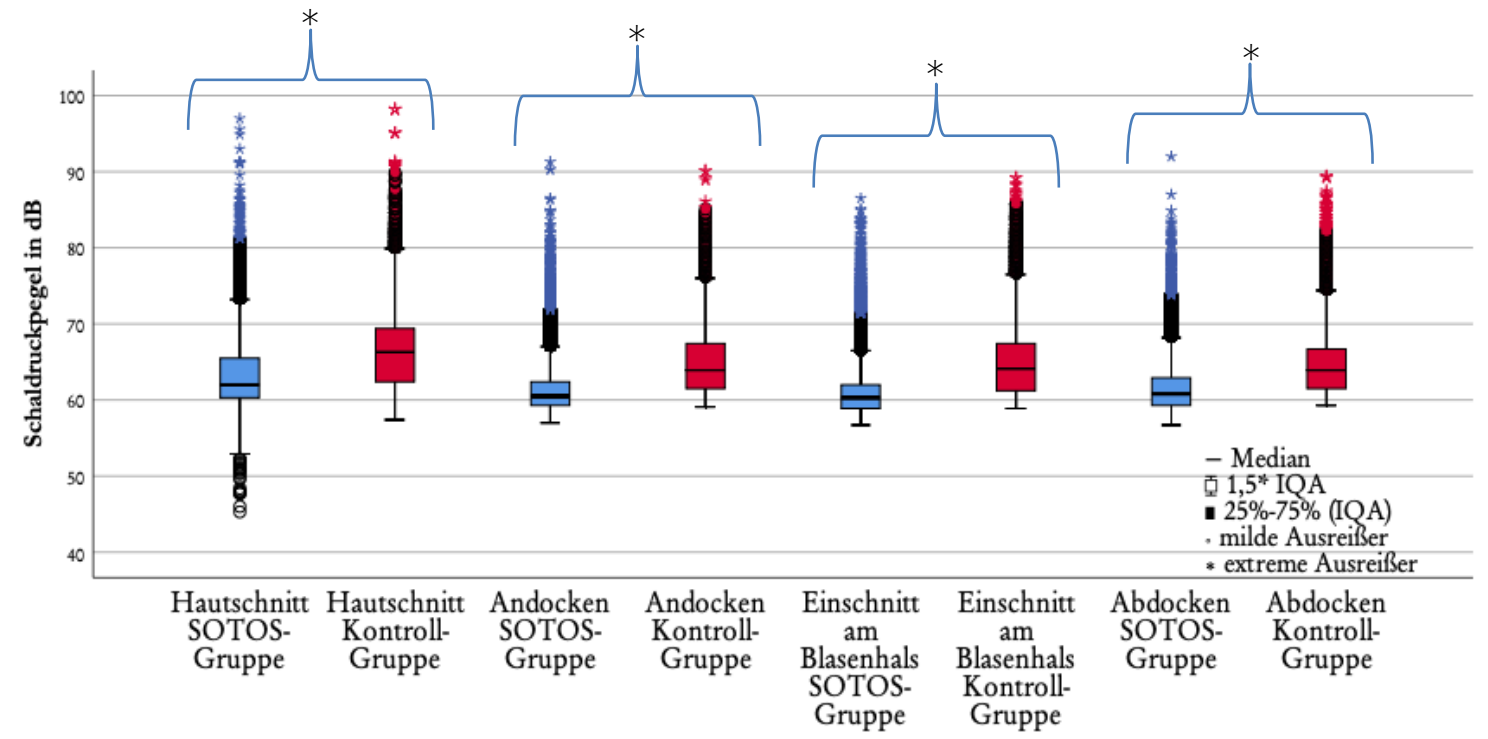

Abbildung 23: Vergleich der gemessenen Schalldruckpegel (in dB) am OP-Feld (mit $1 \mathrm{~m}$ Abstand) zu vier verschiedenen Messzeitpunkten (+/- $10 \mathrm{~min})$ für die beiden Studienarme (IQA= Interquartilsabstand, * statistisch signifikant).

Es wurden für die Abbildung jeweils alle Messzeitpunkte der insgesamt 32 Messtage für die SOTOS- und die Kontroll-Gruppe in die Analyse einbezogen. Somit ergaben sich für die einzelnen Messzeitpunkte insgesamt 19.200 Schalldruckpegel (jeweils ein gemessener Schalldruckpegel pro Sekunde für einen Zeitraum von 20 Minuten). Es zeigte sich eine signifikante Reduktion des Schalldruckpegels in der SOTOS-Gruppe gegenüber den Kontrollen für jeden Messzeitpunkt (Tabelle 8).

Tabelle 8: Vergleich der gemessenen Schalldruckpegel (in dB) am OP-Feld (mit $1 \mathrm{~m} \mathrm{Ab-}$ stand) zu vier verschiedenen Messzeitpunkten (+/- $10 \mathrm{~min}$ ) für die beiden Studienarme (Mittelwert \pm Standardabweichung; $*$ Wilcoxen rank sum Test).

\begin{tabular}{l|llll} 
Messzeitpunkt & SOTOS-Gruppe & Kontroll-Gruppe & Differenz & $p$-Wert \\
\hline Hautschnitt & $63,35( \pm 4,64)$ & $66,54( \pm 5,24)$ & $-3,19$ & $\mathrm{p}<0,001 *$ \\
Andocken & $61,49( \pm 3,64)$ & $64,86( \pm 4,09)$ & $-3,37$ & $\mathrm{p}<0,001 *$ \\
Einschnitt Blasenhals & $61,24( \pm 3,71)$ & $65,12( \pm 4,62)$ & $-3,88$ & $\mathrm{p}<0,001 *$ \\
Abdocken & $61,79( \pm 3,75)$ & $64,79( \pm 4,39)$ & -3 & $\mathrm{p}<0,001 *$
\end{tabular}

Der Schalldruckpegel (in dB), gemessen an der Konsole in beiden Studiengruppen für die jeweiligen Messzeitpunkte, wird in Abbildung 24 gezeigt. 


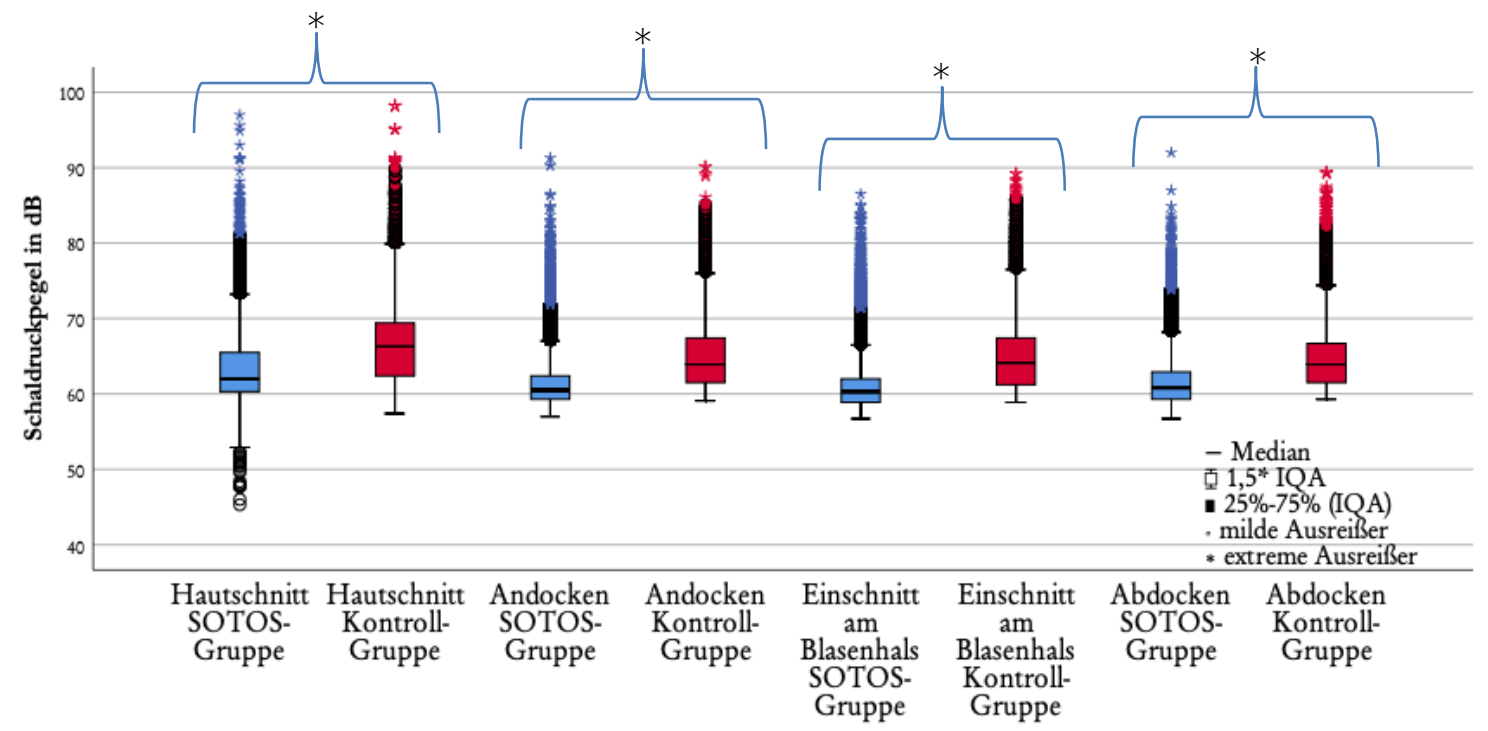

Abbildung 24: Vergleich der gemessenen Schalldruckpegel (in dB) an der Konsole (mit $1 \mathrm{~m}$ Abstand) zu vier verschiedenen Messzeitpunkten (+/- 10 min) für die beiden Studienarme (IQA= Interquartilsabstand, * statistisch signifikant).

Auch in der Analyse der Schalldruckpegel an der Konsole zeigte sich zu den vier Messzeitpunkten jeweils eine signifikante Reduktion in der SOTOS-Gruppe gegenüber der Kontrollgruppe (Tabelle 9).

Tabelle 9: Vergleich der gemessenen Schalldruckpegel (in $\mathrm{dB}$ ) an der Konsole (mit $1 \mathrm{~m}$ Abstand) zu vier verschiedenen Messzeitpunkten (+/- $10 \mathrm{~min}$ ) für die beiden Studienarme (Mittelwert \pm Standardabweichung; $*$ Wilcoxen rank sum Test) mit der Differenz zwischen Kontroll- und SOTOS-Gruppe.

\begin{tabular}{l|llll} 
Messzeitpunkt & SOTOS-Gruppe & Kontroll-Gruppe & Differen₹ & p-Wert \\
\hline Hautschnitt & $62,08( \pm 4,41)$ & $65,83( \pm 4,52)$ & $-3,75$ & $\mathrm{p}<0,001 *$ \\
Andocken & $61,22( \pm 3,44)$ & $65,29( \pm 4,57)$ & $-4,07$ & $\mathrm{p}<0,001 *$ \\
Einschnitt Blasenhals & $61,17( \pm 3,42)$ & $64,85( \pm 4,39)$ & $-3,68$ & $\mathrm{p}<0,001 *$ \\
Abdocken & $62,16( \pm 4,20)$ & $65,43( \pm 4,83)$ & $-3,27$ & $\mathrm{p}<0,001 *$
\end{tabular}

In der folgenden Analyse erfolgte eine Umrechnung des gemessenen Schalldruckpegels in den Schalldruck für die vier Messzeitpunkte. Es wurden erneut alle Messzeitpunkte der insgesamt 32 Messtage für die SOTOS-Gruppe und die Kontroll-Gruppe einbezogen. 


\subsubsection{Messergebnisse des Schalldruckes an vier verschiedenen Messzeitpunkten}

In der Abbildung 25 wird der Schalldruck (in $\mathrm{mPa}$ ) am OP-Feld (mit $1 \mathrm{~m}$ Abstand) zu den jeweiligen Messzeitpunkten für beide Studienarme gegenübergestellt. Zur besseren Darstellung erfolgte die Darstellung der X-Achse in einer logarithmischen Skala.

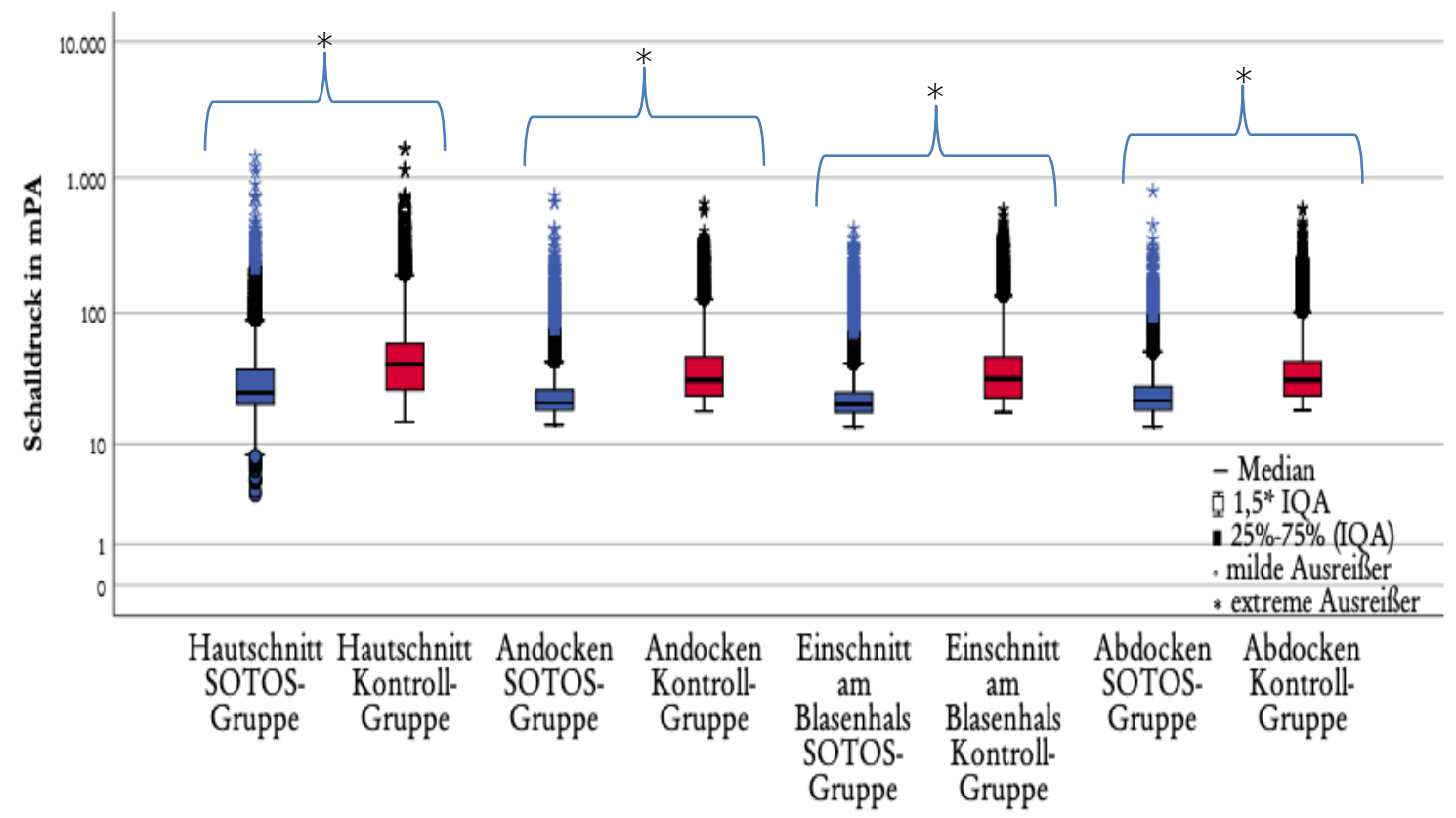

Abbildung 25: Vergleich des Schalldrucks (in $\mathrm{mPa}$ ) gemessen am OP-Feld (mit $1 \mathrm{~m} \mathrm{Ab}$ stand) zu den vier verschiedenen Messzeitpunkten (+/- $10 \mathrm{~min}$ ) während der Operation zwischen den beiden Studienarmen (IQA = Interquartilsabstand, $*$ statistisch signifikant).

Es zeigte sich eine signifikante Senkung des Schalldruckes in der SOTOS-Gruppe gegenüber der Kontroll-Gruppe (Tabelle 10).

Tabelle 10: Vergleich des gemessenen Schalldrucks (in $\mathrm{mPa}$ ) am OP-Feld (mit $1 \mathrm{~m} \mathrm{Ab-}$ stand) zu vier verschiedenen Messzeitpunkten (+/- $10 \mathrm{~min}$ ) für die beiden Studienarme (Mittelwert \pm Standardabweichung; $*$ Wilcoxen rank sum Test) mit der Differenz zwischen Kontroll- und SOTOS-Gruppe.

\begin{tabular}{l|llll} 
Messzeitpunkt & SOTOS-Gruppe & Kontroll-Gruppe & Differen 2 & p-Wert \\
\hline Hautschnitt & $35,59( \pm 36,09)$ & $52,94( \pm 52,25)$ & $-17,35$ & $\mathrm{p}<0,001 *$ \\
Andocken & $26,80( \pm 20,92)$ & $40,22( \pm 29,58)$ & $-13,42$ & $\mathrm{p}<0,001 *$ \\
Einschnitt Blasenhals & $26,21( \pm 20,44)$ & $43,29( \pm 37,28)$ & $-17,08$ & $\mathrm{p}<0,001 *$ \\
Abdocken & $27,80( \pm 20,07)$ & $40,78( \pm 33,54)$ & $-12,98$ & $\mathrm{p}<0,001 *$
\end{tabular}


Gegenüberstellend wird in Abbildung 26 der Schalldruck (in $\mathrm{mPa}$ ) an der Konsole zu den jeweiligen Messzeitpunkten für die beiden Studienarme dargestellt.

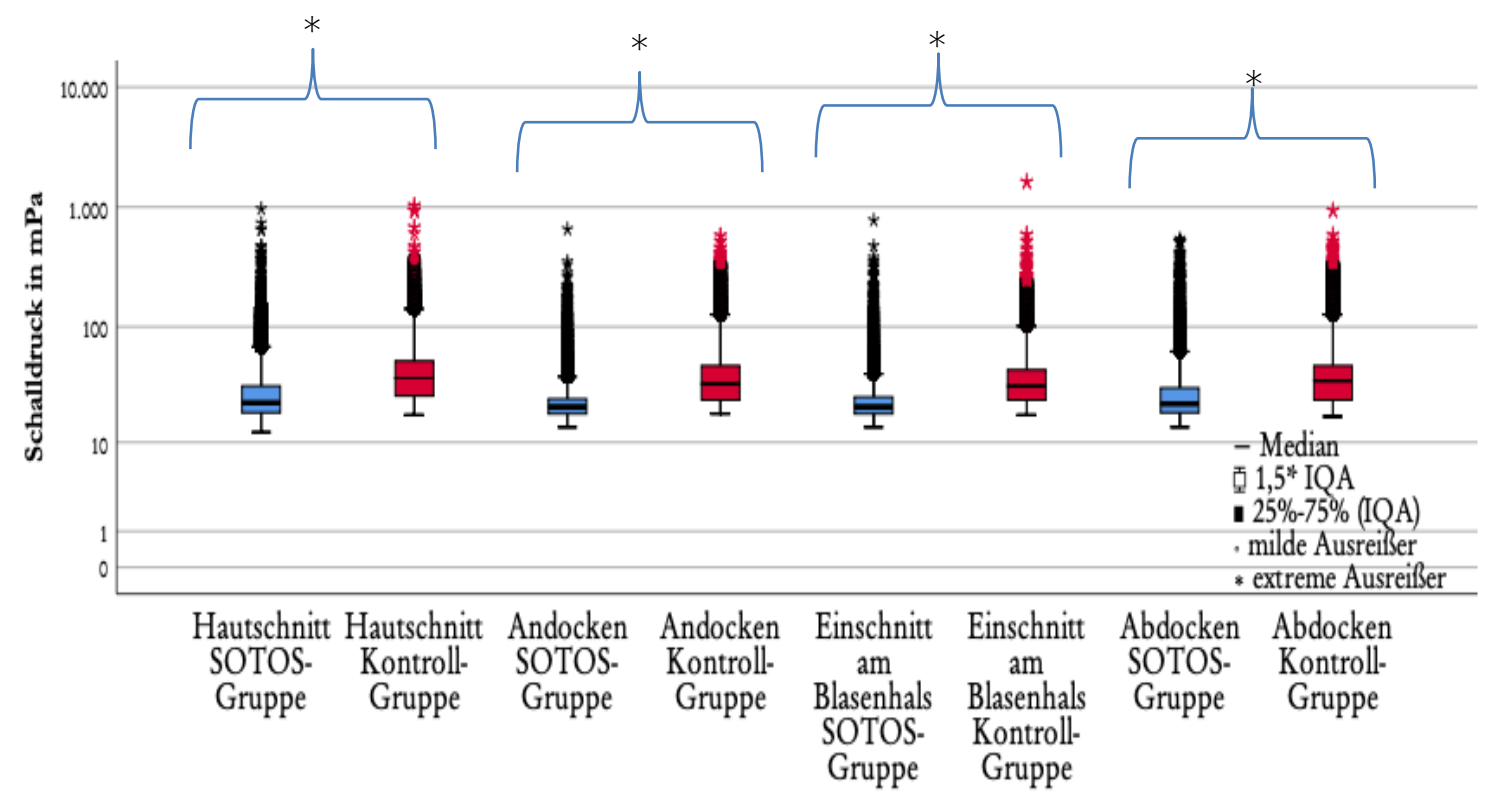

Abbildung 26: Vergleich des Schalldrucks (in $\mathrm{mPa}$ ) gemessen an der Konsole zu vier verschiedenen Messzeitpunkten (+/- 10 min) während der Operation zwischen den beiden Studienarme (IQA = Interquartilsabstand, * statistisch signifikant).

Tabelle 11: Vergleich des gemessenen Schalldrucks (in $\mathrm{mPa}$ ) an der Konsole (mit $1 \mathrm{~m} \mathrm{Ab}$ stand) $\mathrm{zu}$ vier verschiedenen Messzeitpunkten (+/- $10 \mathrm{~min}$ ) für die beiden Studienarme (Mittelwert \pm Standardabweichung; $*$ Wilcoxen rank sum Test) mit der Differenz zwischen Kontroll- und SOTOS-Gruppe.

\begin{tabular}{l|lccl} 
Messzeitpunkt & SOTOS-Gruppe & Kontroll-Gruppe & Differen 2 & p-Wert \\
\hline Hautschnitt & $30,17( \pm 27,61)$ & $45,96( \pm 37,32)$ & $-15,79$ & $\mathrm{p}<0,001 *$ \\
Andocken & $25,56( \pm 17,19)$ & $43,92( \pm 37,16)$ & $-18,36$ & $\mathrm{p}<0,001 *$ \\
Einschnitt Blasenhals & $25,50( \pm 18,82)$ & $41,41( \pm 37,54)$ & $-15,91$ & $\mathrm{p}<0,001 *$ \\
Abdocken & $30,25( \pm 28,17)$ & $45,57( \pm 41,05)$ & $-15,32$ & $\mathrm{p}<0,001 *$
\end{tabular}

Zusammenfassend zeigte sich sowohl an der Konsole als auch am OP-Feld eine signifikante Reduktion des Schalldrucks in der SOTOS-Gruppe gegenüber der Kontrollgruppe zu allen Messzeitpunkten (Tabelle 10 und 11). 


\subsection{Zusammenfassung der Ergebnisse}

In der dargestellten Untersuchung konnten wir im Vergleich der klinisch-onkologischen Patientendaten sowie der Operationsparameter zeigen, dass die SOTOS- und die Kontrollgruppe vergleichbare Kohorten darstellen. Zudem zeigte sich im vorliegenden Kollektiv, dass das SOTOS keinen Einfluss auf die relevanten Operationsparameter, wie z. B. die OPDauer, Komplikationen und onkologische Ergebnisse wie Resektionsstatus hat.

In den EKG-Messungen war in einzelnen Subgruppen, wie Operateur und Springer, eine tendenzielle, teilweise auch statistisch signifikante Senkung der Herzfrequenz durch den Einsatz von SOTOS zu erkennen. Ein statistisch signifikanter Effekt für das komplette OP-Team konnte jedoch nicht nachgewiesen werden.

Die Lautstärkemessungen wiesen über die gesamte Operation und auch an den vier OPrelevanten Zeitpunkten im Vergleich eine statistisch signifikante Reduktion der Schalldruckpegel und der berechneten Schalldrücke durch das SOTOS nach. 


\section{Diskussion}

Eine umfangreiche Untersuchung zum Einsatz SOTOS in der operativen Urologie wird hier erstmalig vorgestellt. Die Untersuchung erfolgte am Beispiel der radikalen Prostatektomie mit dem Da-Vinci-Operationssystem. Es wurde eine prospektiv geplante randomisierte Studie zur Untersuchung der Stress- und Lärmbelastung während der Operation durchgeführt. Unsere Studie konnte dazu einerseits wegweisende Ergebnisse zeigen, andererseits zeigten sich auch Limitationen. Zusammenfassend konnten wir einen günstigen Effekt auf die Lärmbelastung während der Operation feststellen, jedoch blieb ein statistisch relevanter Effekt auf eine mögliche Verminderung der Stressbelastung für das gesamte OPTeam durch SOTOS in unserer Untersuchung aus. Im Weiteren werden die Ergebnisse diskutiert und zu der relevanten Literatur in Bezug gesetzt.

\subsection{Vergleichbarkeit der klinisch-onkologischen Daten und der Operationsparameter}

Die je 16 Operationen in den zwei Studienarmen bestanden aus vergleichbaren Kollektiven. Die vergleichbaren klinisch-onkologischen Daten (z. B. Tumorcharakteristika, Voroperationen) beider Gruppen basieren sicherlich auf der Indikationsstellung für eine radikale laparoskopische roboterassistierte Prostatovesikulektomie und deren Durchführung.

Eine radikale Prostatektomie ist bei Patienten mit einem lokal begrenzten Karzinom aktuell neben der Radiatio der Goldstandard zur onkologischen Therapie (Mottet et al. 2017). Vor der Operation erfolgte eine Selektion des gesamten Kollektivs hinsichtlich der onkologischen Situation. Somit wurde präoperativ eine onkologisch vergleichbare Verteilung der Patientengruppen hinsichtlich des Gleason-Score, Alter, iPSA und Klassifikation nach D'Amico erreicht und eine homogene Voraussetzung zur Analyse bzw. zum Vergleich der Gruppen erzielt.

Zudem wurde eine blinde Randomisierung der Studie in die SOTOS- und KontrollGruppe durchgeführt. Die Operation wurde nach dem in der Einleitung beschriebenen Verfahren und dem Klinikstandard für jede der 32 Operationen gleich durchgeführt. Es erfolgte zu fast gleichen Anteilen ein Nerverhalt (elf Patienten in der SOTOS- und zehn Patienten in der Kontroll-Gruppe), welcher einen Einfluss auf die Operation, die Operationsdauer und das Stressempfinden haben konnte, da ein intraoperativer Schnellschnitt durchgeführt wird. Dies führt zu erhöhten OP-Zeiten und oft ist eine Operation mittels Nerverhalt operativ aufgrund eines erhöhten Präparationsaufwands des Gefäßnervenbündels aufwendiger. In unserer Untersuchung zeigte sich ein statistisch signifikanter Unterschied der OP-Dauer (mit Nerverhalt im Mittel 4 h 29 min +/- 39min und ohne Nerverhalt 3 h $44 \mathrm{~min}$ +/- $53 \mathrm{~min} ; \mathrm{p}=0,011$ ). 
In beiden Studienarmen war die Prostatagröße (42,7 $\mathrm{ml}$ in der SOTOS- und 47,2 $\mathrm{ml}$ in der Kontroll-Gruppe, präoperativ im TRUS gemessen) vergleichbar. Somit war ein weiterer Faktor mit möglichem Einfluss auf die Operationskomplexität und -dauer durch eine erhöhte Operationsschwierigkeit durch eine große Prostatagröße in beinen Gruppen identisch. Bei einem gesunden jungen Mann liegt die Größe der Prostata zwischen 20-30 ml. Somit waren die Drüsen insgesamt vergrößert, was altersadaptiert als normal gelten kann.

Die Operationsdauer betrug in der Kontrollgruppe im Median 4 h 20 min, in der SOTOSGruppe 4 h 6 min. Dies ist mit den in der Literatur zu findenden Angaben von Operationszeiten zwischen vier bis fünf Stunden im Mittel vergleichbar (Kang et al. 2013). Obwohl keine Signifikanz vorliegt, lässt sich eine Tendenz zu kürzeren Operationszeiten bei SOTOS-Anwendung erkennen. Dies könnte durch die direkte Kommunikation und damit einhergehenden verminderten Nachfragen bzw. Verzögerungen erklärbar sein. Die Installierung des Systems, das Anlegen der Kopfhörer und Mikrofone hatte in dieser Untersuchung keinen negativen Einfluss auf die Operationsdauer gezeigt und erzeugte keine relevante Verzögerung. Dies spricht für die Einfachheit des Systems. Das SOTOS System war in der Klinik schon seit einigen Monaten in Verwendung. Somit war die Eingewöhnungsphase in die Bedienung des Systems und die Verwendung während der Operationen in unserer Untersuchung im Jahre 2017 bereits vollzogen und musste dementsprechend nicht berücksichtigt werden.

Zudem ist bei einer OP-Länge von über vier Stunden im Median ein Zeitaufwand von geschätzt zehn Minuten für das System womöglich nicht relevant. Man könnte weitere Untersuchungen bei Operationen mit geringerer Dauer wie z. B. radikale retroperitoneale Nephrektomie vornehmen und untersuchen, ob dort ein solches System einen Einfluss auf die Dauer der Operation hätte. Zudem wären bei einer Neuanschaffung des Systems eine Evaluierung möglicher Lernkurven und deren Einfluss auf die Operationen vorzunehmen.

Während der radikalen Prostatektomie können schwierige OP-Bedingungen wie vermehrte Blutungen, Verwachsungen durch Voroperationen oder fragliche lokal fortgeschrittene Tumore auftreten. Diese traten in der SOTOS-Gruppe in fünf Fällen (31\%) und bei der Kontrollgruppe in sechs Fällen (37\%) auf. Somit konnte eine derartige Beeinflussung der Operationen im positiven wie im negativen Sinn ausgeschlossen werden.

Postoperativ zeigte sich bei der pathologischen Klassifizierung der untersuchten Präparate ein gewisses Upgrading (37\% in der SOTOS- und 50\% der Patienten in der KontrollGruppe). Dies ist jedoch ein in der Literatur bekannter Effekt. Es ist erwiesen, dass sich der endgültige histologische Befund im Prostatektomie-Präparat von der initialen StanzPathologie unterscheidet (Dinh et al. 2015). Es zeigte sich, dass die präoperativ durchgeführte Prostata-Stanzbiopsie, welche in der Regel mittels systematischer transrektaler 12fach Stanzbiopsie durchgeführt wird, nicht die komplette Prostata und damit die mögliche Tumorbiologie (Gleason-Score und Ausdehnung) der gesamten Drüse abbildet. Somit werden oftmals in der feingeweblichen Untersuchung des endgültigen Prostatapräparates 
ein höherer Gleason-Score oder Zweittumore im Organ festgestellt (Mottet et al. 2017). Neue Verfahren, wie die MRT-TRUS-Fusionsbiopsie der Prostata, könnten diesen Effekt verringern. Mittels multiparametrischer MRT des Beckens werden mögliche Läsionen in der Prostata mittels einer standardisierten Einteilung von eins bis fünf aufsteigend nach dem möglichen Malignitätsgrad nach PIRADS-2 verifiziert (Barentsz et al. 2016). Eine anschließend durchgeführte Fusion der MRT-Bilder mit einem transrektalem Ultraschallbild während der Biopsie ermöglicht, neben einer systematischen Biopsie der Prostata eine gezielte Biopsie der im MRT auffälligen Läsionen (Sonn et al. 2014). Es zeigte sich, dass mittels dieses neuen Verfahrens eine oftmals genauere Detektion der Prostatakarzinome möglich ist (Radtke et al. 2016). Somit könnte dieser Effekt des „Upgradings“ in Zukunft verringert werden.

Aus onkologischer Sicht zeigten sich die Operationen in beiden Gruppen vergleichbar. Bei keinem der Patienten lag eine nodal positive Situation nach obligatorischer systematischer pelviner Lymphadenektomie vor. Eine leichte Häufung von positiven Schnitträndern (R1Situation) bei den Patienten der Kontrollgruppe konnte registriert werden. Ob dies durch die Verwendung des SOTOS und der möglichen engeren Team-Zusammenarbeit resultiert, bleibt spekulativ, ist aber rational nicht erklärbar. Dieser Aspekt könnte im Fokus neuer Studien mit einem größeren Kollektiv liegen.

Ein weiterer wichtiger Aspekt bei der Verwendung eines solchen Systems im Operationssaal ist das mögliche Auftreten von Infektionen nach der Operation. In einer Untersuchung von Shigemura et al. (2014) sind die Infektionsraten bei roborterassistierten Operationen ohnehin geringer als bei offen radikalen Prostatektomien. In unserer Untersuchung führte der Einsatz von SOTOS zu einer höheren, aber nicht signifikanten Häufung von Infektionen. Es zeigte sich eine Verteilung von drei Infektionen vs. eine Infektion in den beiden Armen der Studie. Im weiteren Verlauf könnte eine Umstellung auf ein komplett kabelloses System erwogen werden, um eine weitere Verbesserung der Infektionsrate zu evaluieren. Grundsätzlich wird durch eine effizientere Kommunikation die Notwendigkeit für Gespräche reduziert. Tschan et al. (2015) konnten zeigen, dass eine vermehrte Kommunikation vor allem zu nicht OP-bezogenen Themen eine erhöhte Wundinfektionsrate bei Patienten zeigte. In unserer Untersuchung zeigte sich zwar eine Tendenz für eine erhöhte Infektionsrate in der SOTOS-Gruppe, welche jedoch statistisch insignifikant blieb. Jedoch ist bei den geringen Fallzahlen eine abschließende Wertung nicht möglich. Weitere Untersuchungen im Hinblick auf die postoperativen Infektionen wie Wundinfektionen bei den SOTOSPatienten könnten dieses Thema weiter evaluieren.

Im weiteren postoperativen Verlauf der Patienten zeigte sich eine leichte, jedoch nicht statistisch signifikante Tendenz zu Ungunsten der SOTOS-Gruppe hinsichtlich der postoperativen Komplikationen wie einer Hämatombildung und der Bildung von Lymphozelen. Hier könnten beispielsweise auch die abdominellen Voroperationen der Patienten, welche in der SOTOS-Gruppe signifikant häufiger waren, eine Rolle im postoperativen Verlauf der 
Patienten gespielt haben. Dies ist abschließend nicht zu klären, hatte aber ansonsten keinen Einfluss auf die postoperativen Ergebnisse. Die Patienten zeigten eine vergleichbare mediane Liegedauer in unserer Klinik. Dies könnte auch durch unser standardisiertes postoperatives Management bedingt sein.

Zusammenfassend zeigen die statistischen Vergleiche der beiden Operationsgruppen, dass tendenziell Vorteile hinsichtlich einer verkürzten Operationsdauer und einer besseren onkologischen Resektion (R-Status) durch Anwendung von SOTOS bei der radikalen laparoskopischen roboterassistierten Prostatovesikulektomie zu verzeichnen sind.

\subsection{Auswirkungen auf die Stressbelastung der Akteure im Operationssaal}

Eine weitere zentrale Fragestellung dieser Arbeit war die mögliche Stressbelastung des Operationsteams während der Operation und ob diese durch das SOTOS vermindert werden kann. Speziell interessierten uns die Auswirkungen der Stress- und somit Arbeitsbelastung auf die Herzfunktion. Hierfür führten wir bei allen Beteiligten Messungen mittels Langzeit-EKG während der Operationen durch. Die Auswertung erfolgte durch die Studien-Abteilung der Klinik für Kardiologie und Pneumologie der Universitätsmedizin Göttingen.

Es wurden die minimalen, maximalen und mittleren Werte der Herzfrequenz der OPTeilnehmer verglichen. Diese Werte wurden in der Kontroll- und SOTOS-Gruppe untersucht und gegenübergestellt. Ein statistisch relevanter Unterschied der Herzfrequenzen konnte im Ergebnis zwischen den beiden Gruppen nicht festgestellt werden.

Auch innerhalb des Teams (Operateur, Assistent, instrumentierende Fachkraft und Springer) lagen keine signifikanten Unterschiede vor. Die mittleren Werte lagen bei 86 bzw. 87 Herzschlägen pro Minute. Somit konnte kein Vorteil für die Verwendung des Systems auf die Stressentlastung des Operationsteams anhand der EKG-Herzfrequenz-Messungen gezeigt werden. Einzig in einer Subgruppen-Analyse, den Springer im Saal betreffend, zeigte sich eine statistisch signifikante Reduktion der maximalen Herzfrequenz in der SOTOSGruppe gegenüber der Kontroll-Gruppe (Mittelwert für SOTOS: 111,6 \pm 12 Schläge/min, vs. Kontrolle: 143,8 \pm 35 Schläge/min, $\mathrm{p}<0,001)$. Dies könnte durch eine bessere und direktere Kommunikation mit dem Operateur an der Konsole und dem sterilen OP-Team erklärbar sein, da durch das SOTOS eine klarere Verständigung und somit eine Entlastung des Springers durch Vermeidung von unnötigen Wegen bzw. Nachfragen erzielt wird. So könnte es zu einer gewissen Stressreduktion, dargestellt in Form einer Verminderung von hohen Herzfrequenzen, kommen. Andere Studien hatten diesen klaren Zusammenhang zwischen erhöhter Herzfrequenz im Operationssaal und Stress gezeigt. Sie zeigten, dass Operateure unter kognitivem Stress eine erhöhte Herzfrequenz zeigten (Dimitriev und Saperova 2015; Giannoglou et al. 2008; Jones et al. 2015; McDuff et al. 2014). Einen gewissen Einfluss auf dieses Ergebnis könnte das unterschiedliche Bewegungsprofil des Sprin- 
gers gegenüber dem restlichen Operationsteam haben. Der Operateur, Assistent und die instrumentierende Fachkraft haben eine stationäre Position oder einen sehr geringen Bewegungsradius gegenüber dem Springer. Somit könnte die Auswirkung beim Springer durch die Verwendung von SOTOS erklärbar sein.

Ein weiterer möglicher Grund für unsere Ergebnisse sind die Anzahl der verschiedenen Teilnehmer, die Variabilität der Stressbelastung der Teilnehmer und die Artefakte während der Messung. Durch die Anzahl der Teilnehmer kann es zu Unterschieden in der Messung kommen. Zudem können die unterschiedliche Konstitution, Vorerkrankungen und der Trainingszustand der Probanden zur Verfälschung der Effekte geführt haben. Außerdem wurden während der Operation durch die Bewegung Artefakte in den Messungen hervorgerufen. Diese wurden während der Auswertung aus den Protokollen herausgerechnet. Dies führte möglicherweise zu einer Verfälschung der Messung. Bei weiteren Messungen sollte auf eine mögliche Beschränkung der Teilnehmerzahl geachtet werden, um diese Ungenauigkeiten zu vermeiden. Zusätzlich kann man über eine weniger anfällige EKGMessmethode mittels weniger anfälligen Elektroden diskutieren.

Unabhängig von den Vergleichen zwischen den Studiengruppen fielen im Vergleich zwischen den Akteuren des OP-Teams geringe mittlere Herzfrequenzen der Operateure (Mittelwert: 81,3 \pm 7,1 Schläge/min) im Gegensatz zu den Werten der OP-Assistenten (Mittelwert: 87,7 \pm 10,9 Schläge/min), der instrumentierenden Fachkraft (Mittelwert: 86,9 \pm 7,4 Schläge/min) und der Springer (Mittelwert: 90,8 \pm 7,4 Schläge/min) auf. Zudem zeigten die mittleren Herzfrequenzen der einzelnen Operateure (siehe Abbildung 17) während der Operation relativ geringe Schwankungen. Dies ist aufgrund der hohen Verantwortung und der daraus resultierenden Mehrbelastung während einer Operation erstaunlich. Dies könnte ein Hinweis dafür sein, dass Operateure in einer Operation mittels des robotischen Systems (Da-Vinci) eine konstant geringe Stressbelastung zeigen. Moore et al. (2015) konnten in einer Untersuchung zeigen, dass die Verwendung des Da-Vinci- Operationssystems bei den Operateuren im Vergleich zum einfachen laparoskopischen Operieren zu einer Stressverminderung und besseren Durchführung der einzelnen OP-Aufgaben führt. Hiermit könnte bei den Operateuren in unserer Studie durch die Verwendung des DaVinci-Operationssystems eine konstante niedrigere Stressbelastung anzunehmen sein. Somit war möglicherweise ein Effekt auf die Stressbelastung des SOTOS bei dieser Subgruppe nicht zu verzeichnen. Es wäre zu überlegen, ob die Verwendung von SOTOS bei anderen Operationsverfahren wie z. B. offenen Operationen (z. B. radikale Zystektomie) ebenfalls einen Benefit für den Operateur zeigen könnte.

Das subjektive Empfinden des OP-Teams zeigt, dass das SOTOS-System gern während der Operation eingesetzt wird. Dieses subjektive Empfinden könnte durch die verbesserte Kommunikation durch die geschlossene Gesprächsgruppe sowie das Einspielen von Musik erklärbar sein. Hier könnte die Auswertung der Ergebnisse des Institutes für Sozial- und Kommunikationspsycholgie Hinweise geben. 


\subsection{Lärmbelastung im Operationssaal}

Der letzte Teil der Studie betraf die Evaluierung der Lärmbelästung im Operationssaal. Hierfür führten wir an zwei Stellen im Operationssaal Lautstärkemessungen durch: direkt am Operationsgebiet sowie an der Operateurskonsole des Da-Vinci-Operationssystems. Wir konnten zeigen, dass durch den Einsatz des SOTOS eine deutliche Reduktion der Lärmbelästung im Operationssaal während einer Operation hervorgerufen werden kann.

Die dB-Messwerte und $\mathrm{mPa}-M e s s w e r t e$ zeigten in der statistischen Auswertung, dass im Gesamtverlauf der Operation vom Hautschnitt bis zum Abdocken des Roboters eine statistisch signifikante Reduktion der Lärmbelastung im OP-Gebiet und an der Konsole des Da-Vinci-Operationsroboters hervorgerufen wurde.

Die statistisch signifikante Reduktion der Lärmbelastung während der Operation kann durch die Überbrückung der Entfernung des Operateurs an der Konsole zum sterilen OPTeam am Operationstisch durch SOTOS erklärbar sein. Es ist nicht erforderlich durch eine laute Gesprächsführung die Anweisungen an den Assistenten oder die instrumentierende Fachkraft weiterzugeben und die Nachfragen können direkter über das geschlossene Gesprächsfeld erfolgen. Zudem kann das geschlossene Gesprächsfeld, welches durch die Verwendung des SOTOS im OP-Saal erzeugt wird, zu einer Reduktion der Gespräche auBerhalb operationsrelevanter Themen führen. Hier kann das Ziel weiterer Studien sein, gezielt den Einfluss dieses Systems auf die Kommunikationsstruktur und -inhalte während der Operation zu analysieren.

Studien von Conrad et al. (2010) oder Berguer et al. (2001) konnten einen klaren Zusammenhang zwischen der Lärmbelastung und der Leistungsfähigkeit und dem Verlauf der Operation nachweisen. Es kann angenommen werden, dass durch die Verwendung des SOTOS bei Operationen mit dem Da-Vinci-System die Eingriffe günstig durch niedrigere Lärmpegel beeinflusst werden können. Wiederum konnten wir aber in unseren Untersuchungen keinen Hinweis in den EKG-Herzfrequenz-Messungen auf eine signifikante Reduktion der Stressbelastung durch die Lärmreduktion darstellen. Trotz dieses Faktes ist die tendenzielle Reduktion der Lärmbelastung als positiv für die Gesundheit des OP-Teams anzusehen. Diverse Studien hatten einen negativen Effekt von Lärm auf die Gesundheit gezeigt (Babisch 2000; Cabrera und Lee 2000; Falk und Woods 1973; Giannoglou et al. 2008; Herbold et al. 1989).

In der Untersuchung von Babisch (2000) zeigte der Autor einen Zusammenhang von Verkehrslärm und dem kardiovaskulären Risiko. Es stellt sich eine Erhöhung von ischämischen Erkrankungen des Herzens bei einer Lärmbelastung von über 65-70 dB dar. Im Operationssaal kommt es zu einer noch höheren Belastung (bis über 90 dB bzw. $100 \mathrm{mPa}$ ). Somit ist eine Reduktion des Lärms im Operationssaal potenziell von Vorteil für die langfristige gesundheitliche Entwicklung des Operationsteams. 
Des Weiteren ist von Moorthy et al. (2003) gezeigt worden, dass durch eine Lärmreduktion eine Verminderung von Fehlerquoten und eine Verbesserung der Durchführung von OPAufgaben erzielt werden kann. Diese These könnte in unserer Untersuchung durch eine leicht reduzierte OP-Dauer und eine reduzierte R1-Rate bei den Operationen mit SOTOS gestützt werden. Im Gegensatz hierzu zeigte sich in unseren Untersuchungen jedoch in der SOTOS-Gruppe auch eine leichte Erhöhung der postoperativen Komplikationen wie Nachblutungen. Ein positiver Effekt der Lärmreduktion durch SOTOS auf den Operateur ist zusammenfassend trotzdem zu vermuten.

Ein weiterer positiver Aspekt und ein zur Verbesserung der Kommunikation bzw. auch des Operationsergebnisses beitragender Faktor war sicherlich die während der Operation eingespielte Musik für das Operationsteam. Siu et al. (2010) konnten einen positiven Effekt auf die vom Operateur durchgeführten OP-Aufgaben während laparoskopischen roboterassistierten Operationen durch Musik nachweisen. Wir konnten eine leicht reduzierte OPDauer in der SOTOS-Gruppe verzeichnen, was ein Hinweis auf eine mögliche Verbesserung der Leistung des Operateurs sein kann. Dies sollte jedoch in weiteren Studien gezielt untersucht werden.

Hodge und Thompson (1990) zeigten, dass eine Lärmbelastung im Operationssaal die Kommunikation zwischen den Beteiligten nachhaltig beeinträchtigen kann. Somit ist ein positiver Effekt auch bei der Verwendung des SOTOS möglich.

Ein weiterer positiver Aspekt durch das SOTOS ist die Reduzierung von störenden Begleiterscheinungen und Störgeräuschen. Eine Theorie von Miller (1956) beschreibt, dass ein Mensch sieben Informationen gleichzeitig in seinem Kurzzeitgedächtnis verarbeiten kann. Somit kann eine limitierte Aufnahmefähigkeit unseres Gedächtnisses und der möglichen zu verarbeitenden Informationen im zentralen Nervensystem (ZNS) angenommen werden. Die Theorie von Miller ist nicht unumstritten (Rouder et al. 2008). Jedoch verdeutlicht sie die Grenzen der Verarbeitungsfähigkeit des ZNS. Durch die Verminderung von Störgeräuschen im Operationssaal kann die Anzahl von irrelevanten Informationen für die Operation und somit für das Kurzzeitgedächtnis durch SOTOS reduziert werden. Somit kann sich jeder einzelne Operationsteilnehmer potenziell mehr auf seine eigentliche Kernaufgabe fokussieren. Hierdurch ergibt sich möglicherweise eine objektive sowie subjektive Verbesserung des OP-Ablaufes im Operationssaal und des Operationsverlaufes. Dies sollte Aufgabe weiterer Untersuchungen mit Fokus auf die psychologischen oder kognitiven Auswirkungen sein.

\subsection{Limitationen}

Die Studiengröße von insgesamt 32 Operationen in zwei Gruppen stellt eine erste Limitation in Bezug auf die Aussagekraft der Untersuchung dar. Aufgrund der begrenzten Anzahl von Untersuchungen kann nur eine eingeschränkte Aussage bezüglich der Auswirkungen 
des SOTOS auf die durchgeführten radikalen laparoskopischen roboterassistierten Prostatovesikulektomien getroffen werden. Zudem beschränkte sich die Untersuchung auf die Operation eines Krankheitsbildes. Dies wurde zur besseren Vergleichbarkeit in der Methodik angewendet, jedoch limitiert dies die Aussagekraft für andere roboterassistierte Verfahren, zudem kann keine Aussage für andere offene oder endourologische Verfahren getroffen werden. Es sind sicherlich größere Serien mit verschiedenen OP-Techniken und Verfahren im Nachgang zu dieser ersten Studie sinnvoll, um SOTOS für den weiteren Einsatz in der Urologie oder in anderen Fachdisziplinen zu evaluieren.

Eine weitere Limitation ist die Fehleranfälligkeit der EKG-Messung zur Untersuchung der Stressbelastung während der Operation. Die zu erwartenden Ergebnisse in Bezug auf die Stresssenkung durch den Einsatz von SOTOS bei den Operationen blieben aus. Es zeigte sich, dass die Methodik durch eine hohe Anfälligkeit für Artefaktbildung und individuelle Streuung nur eingeschränkte Aussagen zu einem der zentralen Kernpunkte der Fragestellung dieser Arbeit erbrachte. In weiteren Untersuchungen der Stressbelastung sollte eine Beschränkung der Teilnehmer auf eine kleinere Gruppe ohne häufige Wechsel während der Operation angestrebt werden. Dies stellt jedoch wiederum einen Nachteil in Bezug auf eine Verallgemeinerung der Aussagen dar. Ein alternativer Ansatzpunkt wäre ein Wechsel der Messung auf Veränderungen der Hautimpedanz. Diese Methode wurde u. a. von Hecht (2001, S. 193-252) zur Verifizierung einer psychischen Belastung evaluiert. In weiteren Studien könnte dies eine Verbesserung der Aussagekraft hinsichtlich der Stressbelastung erbringen. 


\section{$5 \quad$ Zusammenfassung}

In einer prospektiven und randomisierten Studie führten wir Untersuchungen zum Einsatz des Silent Operating Theatre Optimisation System bei der radikalen laparoskopischen roboterassistierten Prostatovesikulektomie an unserer Klinik durch.

Die vorliegende Studie umfasste zwei Untersuchungsgruppen, die operativ mit und ohne SOTOS tätig waren.

In beiden Gruppen zeigten sich bezüglich Operationsdauer, intraoperativem Schwierigkeitsgrad, Radikalität, Komplikationen sowie postoperativen Infektionen und Liegedauer keine signifikanten Unterschiede. In der SOTOS-Gruppe war ein Trend zu kürzeren Operationszeiten und zur optimalen lokalen Tumorkontrolle bei der Operation erkennbar.

Ein Effekt auf die Herzfrequenzen der Teilnehmer und damit auf das Stressempfinden konnte nicht dargestellt werden.

Es wurden ubiquitäre Messungen im Operationssaal und individuelle Messungen am Operationsteam zu bestimmten Operationszeitpunkten bezüglich Lautstärke, Lärmpegel und deren Auswirkungen auf die Teilnehmer durchgeführt. Die Schalldruckpegel und Schalldrücke waren im Vergleich zur Kontrollgruppe in der SOTOS-Gruppe signifikant geringer. Dies zeigt sich über die gesamte Operationsdauer.

Wir konnten zeigen, dass durch den Einsatz von SOTOS bei radikalen laparoskopischen roboterassistierten Prostatovesikulektomien eine durchaus bedeutsame Lärmreduzierung im Operationssaal möglich ist. 


\section{$6 \quad$ Literaturverzeichnis}

Abbou CC, Hoznek A, Salomon L, Olsson LE, Lobontiu A, Saint F, Cicco A, Antiphon P, Chopin D (2001): Laparoscopic radical prostatectomy with a remote controlled robot. J Urol 165, 1964-1966

Allen K, Blascovich J (1994): Effects of music on cardiovascular reactivity among surgeons. JAMA $272,882-884$

Arora S, Sevdalis N, Nestel D, Woloshynowych M, Darzi A, Kneebone R (2010): The impact of stress on surgical performance: a systematic review of the literature. Surgery $\underline{147}$, 318-330

Asimakopoulos AD, Pereira Fraga CT, Annino F, Pasqualetti P, Calado AA, Mugnier C (2011): Randomized comparison between laparoscopic and robot-assisted nerve-sparing radical prostatectomy. J Sex Med $\underline{8}, 1503-1512$

Aydin Y, Kaltenbach M (2007): Noise perception, heart rate and blood pressure in relation to aircraft noise in the vicinity of the Frankfurt airport. Clin Res Cardiol 96, 347-358

Babisch W (2000): Traffic Noise and Cardiovascular Disease: Epidemiological Review and Synthesis Noise. Health $\underline{2}$, 9-32

Barentsz JO, Weinreb JC, Verma S, Thoeny HC, Tempany CM, Shtern F, Padhani AR, Margolis D, Macura KJ, Haider MA (2016): Synopsis of the PI-RADS v2 guidelines for multiparametric prostate magnetic resonance imaging and recommendations for use. Eur Urol $\underline{69}, 41$

Barnes B, Kraywinkel K, Nowossadeck E, Schönfeld I, Starker A, Wienecke A, Wolf U (Hrsg.): Bericht zum Krebsgeschehen in Deutschland 2016, Robert Koch-Institut, Berlin 2016

Barocas DA, Mitchell R, Chang SS, Cookson MS (2010): Impact of surgeon and hospital volume on outcomes of radical prostatectomy. Urol Oncol 28, 243-250

Berguer R, Smith WD, Chung YH (2001): Performing laparoscopic surgery is significantly more stressful for the surgeon than open surgery. Surg Endosc 15, 1204-1207

Bill-Axelson A, Holmberg L, Filen F, Ruutu M, Garmo H, Busch C, Nordling S, Haggman M, Andersson SO, Bratell S, et al. (2008): Radical prostatectomy versus watchful waiting in localized prostate cancer: the Scandinavian prostate cancer group-4 randomized trial. J Natl Cancer Inst 100, 1144-1154

Bill-Axelson A, Holmberg L, Ruutu M, Garmo H, Stark JR, Busch C, Nordling S, Haggman M, Andersson SO, Bratell S, et al. (2011): Radical prostatectomy versus watchful waiting in early prostate cancer. N Engl J Med $\underline{364}, 1708-1717$

Binder J, Kramer W (2001): Robotically-assisted laparoscopic radical prostatectomy. BJU Int $\underline{87}, 408-410$

Boorjian SA, Eastham JA, Graefen M, Guillonneau B, Karnes RJ, Moul JW, Schaeffer EM, Stief C, Zorn KC (2012): A critical analysis of the long-term impact of radical prostatectomy on cancer control and function outcomes. Eur Urol 61, 664-675 
Bostwick DG, Grignon DJ, Hammond ME, Amin MB, Cohen M, Crawford D, Gospadarowicz M, Kaplan RS, Miller DS, Montironi R, et al. (2000): Prognostic factors in prostate cancer. College of American Pathologists Consensus Statement 1999. Arch Pathol Lab Med 124, 995-1000

Bott SR, Birtle AJ, Taylor CJ, Kirby RS (2003): Prostate cancer management: an update on localised disease Postgrad. Med J $\underline{79}, 575-580$

Cabrera IN, Lee MH (2000): Reducing noise pollution in the hospital setting by establishing a department of sound: a survey of recent research on the effects of noise and music in health care. Prev Med $\underline{30}, 339-345$

Conrad C, Konuk Y, Werner P, Cao CG, Warshaw A, Rattner D, Jones DB, Gee D (2010): The effect of defined auditory conditions versus mental loading on the laparoscopic motor skill performance of experts. Surg Endosc 24, 1347-1352

Conrad C, Konuk Y, Werner PD, Cao CG, Warshaw AL, Rattner DW, Stangenberg L, Ott HC, Jones DB, Miller DL, et al. (2012): A quality improvement study on avoidable stressors and countermeasures affecting surgical motor performance and learning. Ann Surg 255, 1190-1194

D'Amico AV, Hui-Chen M, Renshaw AA, Sussman B, Roehl KA, Catalona WJ (2006): Identifying men diagnosed with clinically localized prostate cancer who are at high risk for death from prostate cancer. J Urol $\underline{176}, 11-15$

Dimitriev DA, Saperova EV (2015): Heart rate variability and blood pressure during mental stress. Ross Fiziol Zh Im I M Sechenova 101, 98-107

Dinh KT, Mahal BA, Ziehr DR, Muralidhar V, Chen YW, Viswanathan VB, Nezolosky MD, Beard CJ, Choueiri TK, Martin NE, et al. (2015): Incidence and Predictors of Upgrading and Up Staging among 10,000 Contemporary Patients with Low Risk Prostate Cancer. J Urol 194, 343-349

Eastham JA (2008): Robotic-assisted prostatectomy: is there truth in advertising? Eur Urol $\underline{54}, 720-722$

Epstein JI, Partin AW, Sauvageot J, Walsh PC (1996): Prediction of progression following radical prostatectomy. A multivariate analysis of 721 men with long-term follow-up. Am J Surg Pathol 20, 286-292

Falk SA, Woods NF (1973): Hospital noise--levels and potential health hazards. N Engl J Med 289, 774-781

Friedrich MG, Boos M, Pagel M, Thormann T, Berakdar A, Russo S, Tirilomis T (2017): New technical solution to minimise noise exposure for surgical staff: the 'silent operating theatre optimisation system'. BMJ Innov $\underline{3}$, 196-205

Ganzer R, Franiel T, Köllermann J, Kuru T, Baumunk D, Blana A, Hadaschik B, von Hardenberg J, Henkel T, Köhrmann K-U (2017): Fokale Therapie des Prostatakarzinoms. Urologe 56, 1335-1346 
Giannoglou GD, Chatzizisis YS, Zamboulis C, Parcharidis GE, Mikhailidis DP, Louridas GE (2008): Elevated heart rate and atherosclerosis: an overview of the pathogenetic mechanisms. Int J Cardiol 126, 302-312

Hecht K: Chronopsychobiologische Regulationsdiagnostik zur Verifizierung von funktionellen Zuständen und Dysregulationen. In: Hecht K, König O, Scherf HP (Hrsg.): Emotioneller Stress durch Überforderung und Unterforderung. Schibri Verlag, Berlin, Milow, Strassburg 2001, 193-252

Herbold M, Hense HW, Keil U (1989): Effects of road traffic noise on prevalence of hypertension in men: results of the Luebeck Blood Pressure Study. Soz Praventivmed $\underline{34}$, $19-23$

Hodge B, Thompson JF (1990): Noise pollution in the operating theatre. Lancet $\underline{335}$, 891 894

Jones KI, Amawi F, Bhalla A, Peacock O, Williams JP, Lund JN (2015): Assessing surgeon stress when operating using heart rate variability and the State Trait Anxiety Inventory: will surgery be the death of us? Colorectal Dis $17,335-341$

Kang J, Yoon KJ, Min BS, Hur H, Baik SH, Kim NK, Lee KY (2013): The impact of robotic surgery for mid and low rectal cancer: a case-matched analysis of a 3-arm comparison - open, laparoscopic, and robotic surgery. Ann Surg 257, 95-101

Kattan MW, Eastham J (2003): Algorithms for prostate-specific antigen recurrence after treatment of localized prostate cancer. Clin Prostate Cancer 1, 221-226

Kirschbaum C, Hellhammer DH (1999): Noise and Stress - Salivary Cortisol as a NonInvasive Measure of Allostatic Load. Noise Health 1 , 57-66

Lee JY, Lantz AG, McDougall EM, Landman J, Gettman M, Sweet R, Sundaram CP, Zorn KC (2013): Evaluation of potential distractors in the urology operating room. J Endourol 27, $1161-1165$

Leitlinie Prostata 2019: Karzinom Leitlinienprogramm Onkologie (Deutsche Krebsgesellschaft, Deutsche Krebshilfe, AWMF): Interdisziplinäre Leitlinie der Qualität S3 zur Früherkennung, Diagnose und Therapie der verschiedenen Stadien des Prostatakarzinoms, Langversion 5.1, 2019, AWMF Registernummer: 043/022OL, http://www.leitlinienprogramm-onkologie.de/leitlinien/prostatakarzinom/ (abgerufen am: 29.12.2019)

Lentner C: Heart and circulation. In: Lentner C (Hrsg.) Geigy Scientific Tables. 5. Auflage, Ciba-Geigy, Basel 1990, 187-197

Loeb S, Roehl KA, Helfand BT, Catalona WJ (2008): Complications of open radical retropubic prostatectomy in potential candidates for active monitoring. Urology $\underline{72}, 887-$ 891

Lopez-Beltran A, Mikuz G, Luque RJ, Mazzucchelli R, Montironi R (2006): Current practice of Gleason grading of prostate carcinoma. Virchows Arch $\underline{448}, 111-118$

MacClelland DC (1979): Music in the operating room. AORN J 29, 252-260 
Makarov DV, Trock BJ, Humphreys EB, Mangold LA, Walsh PC, Epstein JI, Partin AW (2007): Updated nomogram to predict pathologic stage of prostate cancer given prostatespecific antigen level, clinical stage, and biopsy Gleason Score (Partin tables) based on cases from 2000 to 2005. Urology $\underline{69}$, 1095-1101

Mayrhofer S (Hrsg.): KLT-R. Konzentrations-Leistungs-Test - Revidierte Fassung. Hogrefe, Göttingen 2001

McDuff D, Gontarek S, Picard R (2014): Remote measurement of cognitive stress via heart rate variability. Conf Proc IEEE Eng Med Biol Soc 2014, 2957-2960

McEwen BS (1998): Protective and damaging effects of stress mediators. N Engl J Med $\underline{338}, 171-179$

Miller GA (1956): The magical number seven, plus or minus two: Some limits on our capacity for processing information. Psychol. Rev $\underline{63}, 81$

Millin T (1945): Retropubic prostatectomy; a new extravesical technique; report of 20 cases. Lancet $\underline{2}$, 693-696

Moore LJ, Wilson MR, Waine E, McGrath JS, Masters RS, Vine SJ (2015): Robotically assisted laparoscopy benefits surgical performance under stress. J Robot Surg 9, 277-284

Moorthy K, Munz Y, Dosis A, Bann S, Darzi A (2003): The effect of stress-inducing conditions on the performance of a laparoscopic task. Surg Endosc 17, 1481-1484

Moorthy K, Munz Y, Undre S, Darzi A (2004): Objective evaluation of the effect of noise on the performance of a complex laparoscopic task. Surgery 136, 25-30; discussion 31

Moris DN, Linos D (2013): Music meets surgery: two sides to the art of "healing". Surg Endosc 27, 719-723

Mosteller F, Tukey JW (Hrsg): Data analysis and regression: a second course in statistics. Addison-Wesley, Boston 1977

Mottet, Bellmunt J, Bolla M, Briers E, Cumberbatch MG, De Santis M, Fossati N, Gross T, Henry AM, Joniau S, et al. (2017): EAU-ESTRO-SIOG Guidelines on Prostate Cancer. Part 1: Screening, Diagnosis, and Local Treatment with Curative Intent. Eur Urol 71, 618629

Nuttall M, van der Meulen J, Phillips N, Sharpin C, Gillatt D, McIntosh G, Emberton M (2004): A systematic review and critique of the literature relating hospital or surgeon volume to health outcomes for 3 urological cancer procedures. J Urol 172, 2145-2152

Partin AW, Mangold LA, Lamm DM, Walsh PC, Epstein JI, Pearson JD (2001): Contemporary update of prostate cancer staging nomograms (Partin Tables) for the new millennium. Urology $\underline{58}, 843-848$

Radtke JP, Schwab C, Wolf MB, Freitag MT, Alt CD, Kesch C, Popeneciu IV, Huettenbrink C, Gasch C, Klein T, et al. (2016): Multiparametric Magnetic Resonance Imaging (MRI) and MRI-Transrectal Ultrasound Fusion Biopsy for Index Tumor Detection: Correlation with Radical Prostatectomy Specimen. Eur Urol 70, 846-853 
Reznick RK (1993): Teaching and testing technical skills. Am J Surg 165, 358-361

Roehl KA, Han M, Ramos CG, Antenor JA, Catalona WJ (2004): Cancer progression and survival rates following anatomical radical retropubic prostatectomy in 3,478 consecutive patients: long-term results. J Urol 172, 910-914

Rouder JN, Morey RD, Cowan N, Zwilling CE, Morey CC, Pratte MS (2008): An assessment of fixed-capacity models of visual working memory. PNAS $\underline{105}$, 5975-5979

Ruurda JP, van Vroonhoven TJ, Broeders IA (2002): Robot-assisted surgical systems: a new era in laparoscopic surgery. Ann R Coll Surg Engl 84, 223-226

Sevdalis N, Healey AN, Vincent CA (2007): Distracting communications in the operating theatre. J Eval Clin Pract 13, 390-394

Shapiro RA, Berland T (1972): Noise in the operating room N Engl J Med 287, 1236-1238

Shigemura K, Tanaka K, Yamamichi F, Muramaki M, Arakawa S, Miyake H, Fujisawa M (2014): Comparison of postoperative infection between robotic-assisted laparoscopic prostatectomy and open radical prostatectomy. Urol Int 92, 15-19

Siu KC, Suh IH, Mukherjee M, Oleynikov D, Stergiou N (2010): The effect of music on robot-assisted laparoscopic surgical performance. Surg Innov 17, 306-311

Sonn GA, Chang E, Natarajan S, Margolis DJ, Macairan M, Lieu P, Huang J, Dorey FJ, Reiter RE, Marks LS (2014): Value of targeted prostate biopsy using magnetic resonanceultrasound fusion in men with prior negative biopsy and elevated prostate-specific antigen. Eur Urol 65, 809-815

Stansfeld SA, Berglund B, Clark C, Lopez-Barrio I, Fischer P, Ohrstrom E, Haines MM, Head J, Hygge S, van Kamp I, et al. (2005): Aircraft and road traffic noise and children's cognition and health: a cross-national study. Lancet $\underline{365}, 1942-1949$

Svanholm H, Mygind H (1985): Prostatic carcinoma reproducibility of histologic grading. Acta Pathol Microbiol Immunol Scand A $\underline{93}$, 67-71

Tooher R, Swindle P, Woo H, Miller J, Maddern G (2006): Laparoscopic radical prostatectomy for localized prostate cancer: a systematic review of comparative studies. J Urol 175, 2011-2017

Trinh QD, Sammon J, Sun M, Ravi P, Ghani KR, Bianchi M, Jeong W, Shariat SF, Hansen J, Schmitges J, et al. (2012): Perioperative outcomes of robot-assisted radical prostatectomy compared with open radical prostatectomy: results from the nationwide inpatient sample. Eur Urol 61, 679-685

Tschan F, Seelandt J, Keller S, Semmer N, Kurmann A, Candinas D, Beldi G (2015): Impact of case-relevant and case-irrelevant communication within the surgical team on surgical-site infection. Br J Surg 102, 1718-1725

Walsh PC, Donker PJ (1982): Impotence following radical prostatectomy: insight into etiology and prevention. J Urol 128, 492-497 
Walsh PC, Lepor H, Eggleston JC (1983): Radical prostatectomy with preservation of sexual function: anatomical and pathological considerations. Prostate $\underline{4}, 473-485$

Wilt TJ, Brawer MK, Jones KM, Barry MJ, Aronson WJ, Fox S, Gingrich JR, Wei JT, Gilhooly P, Grob BM, et al. (2012): Radical prostatectomy versus observation for localized prostate cancer. N Engl J Med 367, 203-213

Wittekind C (Hrsg.): TNM Klassifikation maligner Tumoren. 8. Auflage; Wiley-VCH, Weinheim 2017 


\section{Danksagung}

Mein Dank geht an Prof. Dr. med. Lutz Trojan, Direktor der Klinik für Urologie der Universitätsmedizin Göttingen, für die Überlassung des Themas sowie die umfassende Unterstützung bei dieser Promotion. Zudem bedanke ich mich bei PD Dr. med. Martin Friedrich für die Installation von SOTOS in der Klinik für Urologie und entscheidende technische und konzeptionelle Unterstützung. Für die Kooperation mit der Klinik für Kardiologie und Pneumologie der Universitätsmedizin Göttingen und dem Institut für Sozial- und Kommunikationspsycholgie der Universität Göttingen möchte ich mich zudem in aller Form bedanke 